\title{
(C) OPEN ACCESS \\ British Society of Gastroenterology guidelines on the diagnosis and management of patients at risk of gastric adenocarcinoma
}

\author{
Matthew Banks, ${ }^{\oplus 1,2}$ David Graham, ${ }^{1,3}$ Marnix Jansen, ${ }^{4}{ }^{4}$ Takuji Gotoda,${ }_{1}^{5}$ Sergio Coda, ${ }^{6}$ \\ Massimiliano di Pietro, ${ }^{7,8}$ Noriya Uedo, ${ }^{9}$ Pradeep Bhandari, ${ }^{10}$ D Mark Pritchard, ${ }^{11}$ \\ Ernst J Kuipers, ${ }^{12}$ Manuel Rodriguez-Justo, ${ }^{4}$ Marco R Novelli, ${ }^{4}$ Krish Ragunath, ${ }^{13}$ \\ Neil Shepherd, ${ }^{14}$ Mario Dinis-Ribeiro ${ }^{15}$
}

For numbered affiliations see end of article.

Correspondence to Dr Matthew Banks, University College London Hospital, University College London Hospitals NHS Foundation Trust, London NW12PG, UK: matthew.banks2@nhs.net

Received 16 December 2018 Revised 6 May 2019 Accepted 17 May 2019 Published Online First 5 July 2019
Check for updates

(C) Author(s) (or their employer(s)) 2019. Re-use permitted under CC BY-NC. No commercial re-use. See rights and permissions. Published by BMJ.

To cite: Banks M, Graham D, Jansen $\mathrm{M}$, et al. Gut

2019:68:1545-1575.

\section{ABSTRACT}

Gastric adenocarcinoma carries a poor prognosis, in part due to the late stage of diagnosis. Risk factors include Helicobacter pylori infection, family history of gastric cancer-in particular, hereditary diffuse gastric cancer and pernicious anaemia. The stages in the progression to cancer include chronic gastritis, gastric atrophy (GA), gastric intestinal metaplasia (GIM) and dysplasia. The key to early detection of cancer and improved survival is to non-invasively identify those at risk before endoscopy. However, although biomarkers may help in the detection of patients with chronic atrophic gastritis, there is insufficient evidence to support their use for population screening. High-quality endoscopy with full mucosal visualisation is an important part of improving early detection. Image-enhanced endoscopy combined with biopsy sampling for histopathology is the best approach to detect and accurately risk-stratify GA and GIM. Biopsies following the Sydney protocol from the antrum, incisura, lesser and greater curvature allow both diagnostic confirmation and risk stratification for progression to cancer. Ideally biopsies should be directed to areas of GA or GIM visualised by high-quality endoscopy. There is insufficient evidence to support screening in a low-risk population (undergoing routine diagnostic oesophagogastroduodenoscopy) such as the UK, but endoscopic surveillance every 3 years should be offered to patients with extensive GA or GIM. Endoscopic mucosal resection or endoscopic submucosal dissection of visible gastric dysplasia and early cancer has been shown to be efficacious with a high success rate and low rate of recurrence, providing that specific quality criteria are met.

\section{EXECUTIVE SUMMARY}

Gastric adenocarcinoma continues to be a frequent cause of death in the world and is the 16th most common cancer in the UK. The most common stages in the progression to gastric adenocarcinoma are gastric atrophy (GA) and gastric intestinal metaplasia (GIM), which are collectively known as chronic atrophic gastritis (CAG). These conditions are principally caused by Helicobacter pylori infection and less commonly by autoimmune gastritis. The key to having a significant impact on the prognosis of gastric adenocarcinoma and its economic burden is to accurately identify individuals at greatest risk and intervene with recognised efficacious treatments, including endoscopic resection,before cancer is established. The British Society of Gastroenterology (BSG) endoscopy committee agreed to create a guideline to provide statements and recommendations on the prevalence, risks, diagnosis, treatment, surveillance and screening of gastric premalignant and early gastric malignant lesions. The principal patient group are those found to have GA, GIM, gastric epithelial dysplasia or early gastric adenocarcinoma limited to the mucosal or superficial submucosal layers. The target users include gastroenterologists, GI surgeons, pathologists, endoscopists and general practitioners. We followed the Appraisal of Guidelines for Research and Evaluation (AGREE II) instrument, and the quality of the evidence was assessed according to the Grading of Recommendations Assessment, Development and Evaluation (GRADE) system.

A series of statements, recommendations and suggestions are proposed to ensure that there is consistency of practice, such that patients with gastric premalignant and early gastric malignant lesions are provided with optimal care. These recommendations are listed below:

1. We recommend $H$. pylori eradication to reduce the risk of gastric adenocarcinoma development in patients who have GA (evidence level: high quality; grade of recommendation: high; level of agreement: 100\%).

2. We suggest that $H$. pylori eradication may be of some benefit to reduce the risk of developing gastric adenocarcinoma in those who already have H. pylori-associated GIM, dysplasia or cancer (evidence level: high quality; grade of recommendation: weak; level of agreement: $100 \%)$.

3. We do not recommend the use of biomarkers as a screening tool in areas with a low incidence of gastric adenocarcinoma, such as the UK (evidence level: low quality; grade of recommendation: weak; level of agreement: 93\%).

4. We recommend that patients at higher risk for gastric adenocarcinoma, including $G A$ and GIM, should undergo a full systematic endoscopy protocol of the stomach with clear photographic documentation of gastric regions and pathology. We suggest a minimum examination 
time of 7 min (evidence level: moderate quality; grade of recommendation: strong; level of agreement: 100\%).

5. GAand GIM may be detectable by white light endoscopy (WLE); however, the accuracy is poor. Therefore, we do not recommend establishing a diagnosis or risk stratification using WLE alone (evidence level: moderate quality; grade of recommendation: strong; level of agreement: 93\%).

6. We recommend image-enhanced endoscopy (IEE) as the best imaging modality to accurately detect and risk-stratify GA and GIM (evidence level: moderate quality; grade of recommendation: strong; level of agreement: 100\%).

7. We recommend that endoscopic appearances on WLE suggestive of GA or GIM require escalation to high-resolution IEE and, where available, magnification endoscopy (evidence level: low quality; grade of recommendation: strong; level of agreement: $100 \%)$.

8. We recommend that the location and extent of GA and GIM should be clearly documented with photographic evidence. Endoscopic grading should be documented as distal gastric (affecting antrum or incisura-low risk) or proximal gastric (affecting the corpus with or without the antrum and incisura-high risk) (evidence level: low quality; grade of recommendation: strong; level of agreement: 93\%).

9. We recommend that endoscopic appearances on WLE of gastric dysplasia and early gastric cancer (differences in colour, loss of vascularity, slight elevation or depression, nodularity, thickening, and abnormal convergence or flattening of folds) require escalation to IEE and, where available, magnification endoscopy (evidence level: low quality; grade of recommendation: strong; level of agreement: 100\%).

10. We recommend IEE as the best imaging modality to accurately diagnose and stage gastric dysplasia and early gastric cancer (evidence level: moderate quality; grade of recommendation: strong; level of agreement: 100\%).

11. We recommend that patients with image-enhanced features of CAG should undergo biopsies for confirmation of endoscopic diagnosis; biopsies are directed at mucosal sites within Sydney protocol areas where enhanced imaging discloses GIM. Biopsy samples should be collected in separate containers and labelled as either 'directed' or 'random' to corroborate endoscopic staging assessment (evidence level: low quality; grade of recommendation: strong; level of agreement: 93\%).

12. We suggest that a baseline endoscopy with biopsies should be considered in individuals aged $\geq 50$ years, with laboratory evidence of pernicious anaemia, defined by vitamin $B_{12}$ deficiency and either positive gastric parietal cell or intrinsic factor antibodies. As GA affects the corpus in pernicious anaemia, biopsies should be taken from the greater and lesser curves (evidence level: low quality; grade of recommendation: weak; level of agreement: 93\%).

13. We recommend endoscopic surveillance every 3 years should be offered to patients diagnosed with extensive GA or GIM, defined as that affecting the antrum and body (evidence level: low quality; grade of recommendation: strong; level of agreement: $100 \%$ ).

14. We do not recommend surveillance in patients with GA or GIM limited just to the gastric antrum unless there are additional risk factors, such as a strong family history of gastric cancer or persistent $H$. pylori infection, then we suggest 3-yearly surveillance (evidence level: low quality; grade of recommendation: strong; level of agreement: 93\%).

15. We recommend that patients with non-visible, low-grade dysplasia (LGD) should undergo a second endoscopy with enhanced imaging and extensive biopsy sampling, followed by a repeat endoscopy within 1 year if no visible neoplasia is detected. If there is persistent, non-visible LGD, endoscopy should be repeated annually thereafter (evidence level: low quality; grade of recommendation: strong; level of agreement: $100 \%)$.

16. We recommend that patients with non-visible, high-grade dysplasia (HGD) should undergo an immediate second endoscopy with enhanced imaging and extensive biopsy sampling. We recommend ongoing surveillance at 6-monthly intervals for persistent, non-visible HGD. HGD should be discussed at the regional upper GI cancer multidisciplinary team and referred to a clinician with the appropriate expertise (evidence level: low quality; grade of recommendation: strong; level of agreement: 100\%).

17. We recommend that all gastric dysplasia and early gastric adenocarcinoma should be resected en bloc (an endoscopic mucosal resection (EMR) technique can achieve en bloc excision for lesions $\leq 10 \mathrm{~mm}$ in size, but only an endoscopic submucosal dissection (ESD) technique can ensure en bloc excision for lesions $>10 \mathrm{~mm}$ in size) (evidence level: high quality; grade of recommendation: strong; level of agreement: $100 \%)$.

18. We recommend that complete (RO) endoscopic resection of gastric dysplasia and early gastric adenocarcinoma with the following features should be considered as curative:

1. LGD.

2. HGD.

3. Well or moderately differentiated intramucosal adenocarcinoma, irrespective of size and without ulceration.

4. Well or moderately differentiated intramucosal adenocarcinoma, $<3.0 \mathrm{~cm}$ in size if ulcerated.

5. Well or moderately differentiated submucosal adenocarcinoma, $<3.0 \mathrm{~cm}$ in size, with superficial submucosal invasion (Sm1; <500 um submucosal invasion as measured in a straight line from the deepest fibre of the muscularis mucosae).

6. Poorly differentiated intramucosal adenocarcino$m a, \leq 2.0 \mathrm{~cm}$ in size (evidence level: moderate quality; grade of recommendation: strong; level of agreement: 93\%).

19. The histopathological features of early gastric adenocarcinoma associated with a higher risk of lymph node metastasis (LNM) after endoscopic resection include the following:

1. Poorly differentiated submucosal cancer, irrespective of invasion depth below muscularis mucosae.

2. Signet ring cancer.

3. Lymphovascular invasion.

4. Depth of submucosal invasion $\geq 500 \mu m$ as measured in a straight line from the deepest fibre of the muscularis $m u$ cosae (evidence level: moderate quality; grade of recommendation: strong; level of agreement: 93\%).

20. We do not recommend the use of non-steroidal anti-inflammatory drugs (NSAIDs) or cyclo-oxygenase-2 (COX-2) inhibitors to reduce the risk of progression of premalignant lesions of the stomach (evidence level: moderate; grade of recommendation: strong; level of agreement 100\%).

21. We do not recommend the use of antioxidants as a means to reduce the prevalence of premalignant gastric lesions (evidence level: moderate; grade of recommendation: strong; level of agreement: 100\%).

22. We suggest endoscopic screening should be considered in individuals aged $\geq 50$ years with multiple risk factors for gastric adenocarcinoma (male, smokers, pernicious anaemia)-in 
particular, in those with a first-degree relative with gastric cancer (evidence level: low quality; grade of recommendation: weak; level of agreement: 100\%).

23. We do not recommend endoscopic screening for gastric adenocarcinoma in the UK population (evidence level: low quality; grade of recommendation: strong; level of agreement: $100 \%$ ).

24. We recommend that the number of gastric polyps (or estimated number), location of polyps and size of the largest polyp should be clearly documented (evidence level: low quality; grade of recommendation: strong; level of agreement: $100 \%)$.

25. We recommend that gastric polyps other than fundic gland polyps (FGPs) should be biopsied for histopathological assessment (evidence level: low quality; grade of recommendation: strong; level of agreement: 100\%).

26. We recommend that photographic documentation should be undertaken for all polyps or representative polyps, if numerous (evidence level: low quality; grade of recommendation: strong; level of agreement: 100\%).

27. We recommend that if adenomas or hyperplastic polyps are present, the background mucosa should be endoscopically assessed for GA, GIM, H. pylori and synchronous neoplasia (evidence level: moderate quality; grade of recommendation: strong; level of agreement: 100\%).

28. We recommend that all adenomas should be resected when clinically appropriate and safe to do so (evidence level: low quality; grade of recommendation: strong; level of agreement: 100\%).

29. We recommend that a follow-up gastroscopy should be performed at 12 months after complete endoscopic excision of adenomas, then ongoing surveillance gastroscopy annually thereafter, when appropriate (evidence level: low quality; grade of recommendation: strong; level of agreement: 93\%).

30. We suggest that hyperplastic polyps $>1 \mathrm{~cm}$, pedunculated morphology and those causing symptoms (obstruction, bleeding) should be resected. If present, H. pylori should be eradicated before re-evaluation for endoscopic therapy (evidence level: low quality; grade of recommendation: weak; level of agreement: 100\%).

31. We suggest that enhanced endoscopic imaging is used to aid characterisation of gastric polyps when there is diagnostic uncertainty following white light examination (evidence level: low quality; grade of recommendation: weak; level of agreement: 93\%).

Three pathways have been created to allow a quick reference to the management of CAG, gastric dysplasia and gastric epithelial polyps (Figure 1).

\section{BACKGROUND}

Gastric adenocarcinoma is a major cause of cancer mortality worldwide. ${ }^{1-3}$ In the UK in 2016, there were 5314 cases of gastric cancer, which has been declining gradually with the incidence of $H$. pylori infection. Although there has been a decline in the incidence over the past 50 years, studies have demonstrated an increasing incidence of gastric adenocarcinoma among young white people in the USA, alongside a Swedish study demonstrating an increasing incidence of premalignant gastric lesions among adults aged $35-44$ years. ${ }^{4} 5$ These studies may suggest that historically declining gastric adenocarcinoma incidence rates may alter. At present within the UK, gastric cancer is the 16th most common cancer, within Europe it is the sixth most common cancer, and worldwide it is the fifth most common. ${ }^{1-3}$ Importantly, with the exception of Japan and South Korea, the majority of gastric cancers worldwide are diagnosed at a late stage, resulting in poor prognosis with a $29 \%$ average 5 -year survival. ${ }^{36}$ The UK all-stage average 5-year survival rate is $18 \%$, compared with an $80 \%$ average 5 -year survival for stage $1 \mathrm{~A} .{ }^{3}$ Clearly, Japan's earlier stage of diagnosis and superior 5-year survival highlight the need for earlier recognition and treatment to overcome this bleak prognosis. ${ }^{7}$

The key to having a significant impact on the prognosis of gastric cancer and its global economic burden is to accurately identify the individuals who are at greatest risk and intervene, before gastric cancer development, through recognised efficacious treatments, including endoscopic resection. The canonical model for cancer progression in the stomach is the Correa sequence, which outlines a stepwise linear progression from chronic gastritis through GA, GIM, dysplasia and finally, invasive cancer. ${ }^{8}$ This model was amended to involve the role of $H$. pylori as the prime causative environmental agent ${ }^{9}$ and has remained the dominant model since. Diagnosis and surveillance of higher risk patients in this cascade have been proposed as a strategy to reduce the mortality and morbidity of gastric adenocarcinoma.

The first international guidelines on the management of precancerous conditions and lesions in the stomach (MAPS) were published in 2012 by an international guidelines development group. ${ }^{10}$ These have not yet been revised. There is significant heterogeneity of practice in the management of premalignant and early malignant gastric lesions in the UK, partly because few endoscopists follow MAPS guidance.

\section{OBJECTIVES}

The objectives of these guidelines are to summarise the current evidence and to provide recommendations on the diagnosis, risks and management of gastric premalignant and early malignant lesions, including GA, GIM, dysplasia and early adenocarcinoma. These guidelines are intended for the UK population.

\section{METHODOLOGY}

The guideline was commissioned by the BSG in May 2015 and the proposal approved by Clinical Standards and Services Committee. The guideline was developed in line with the BSG guideline Process. ${ }^{11}$ The purpose of the guideline was to provide guidance to healthcare professionals who are involved in the care of patients at risk of gastric cancer, including gastroenterologists, GI surgeons, pathologists, endoscopists and general practitioners. The AGREE II instrument (May 2009) ${ }^{12}$ was used to guide the methodology. The chair $(\mathrm{MB})$ convened a guideline development group (GDG), consisting of clinicians and patients with experience of, or in the management of, these conditions. Members of the GDG were selected to ensure the relevant professional bodies and specialties were represented, including gastroenterologists, surgeons and pathologists. GDG members were required to declare any conflict of interests. The scope and purpose of the guidelines were initially discussed by the GDG on 20 July 2015 at the first consensus meeting, following which a set of key questions were created. The key questions were subdivided into clinical groups, including diagnosis, treatment, surveillance, screening and research (table 1). The GDG then voted anonymously on the relevance of each question to the guidelines and those with $<75 \%$ agreement were excluded. The GDG then met in London on 17 September 2015 for a second consensus meeting to discuss the agreed key questions, agree on methodology, and allocate leads and subgroups to each question. 


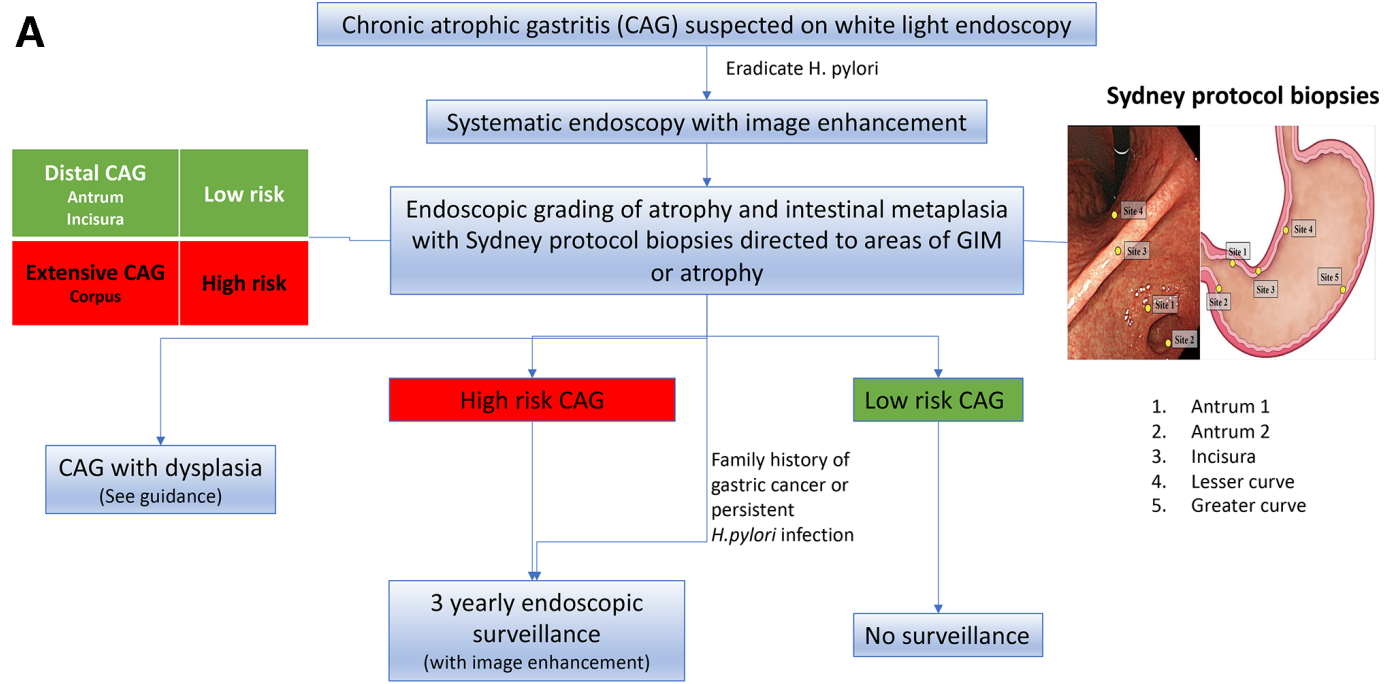

B
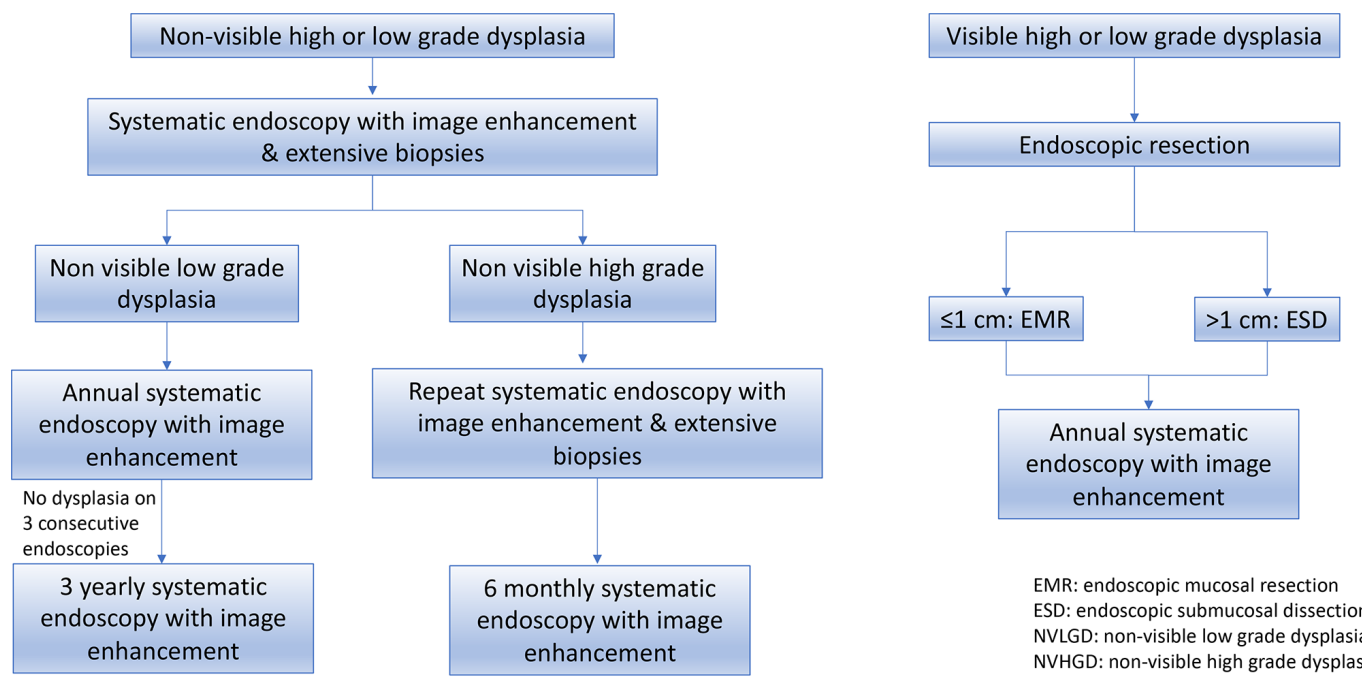

EMR: endoscopic mucosal resection ESD: endoscopic submucosal dissection NVLGD: non-visible low grade dysplasia NVHGD: non-visible high grade dysplasia

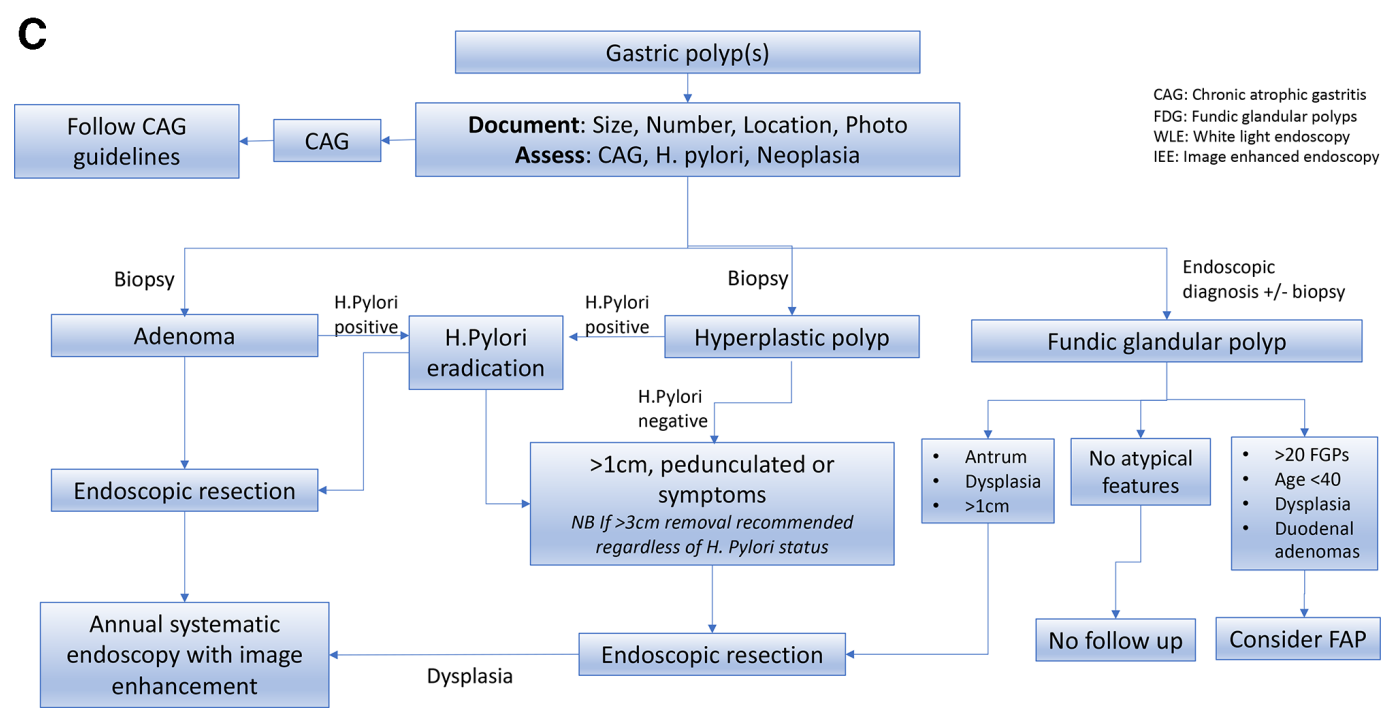

Figure 1 (A) British Society of Gastroenterology (BSG) guidelines for the endoscopic management of chronic atrophic gastritis. (B) BSG guidelines for the endoscopic management of gastric dysplasia. (C) BSG guidelines for the endoscopic management of gastric epithelial polyps. CAG, chronic atrophic gastritis; FAP, familial adenomatous polyposis; FGP, fundic gland polyp; GIM, gastric intestinal metaplasia. 
Table 1 Key questions, subgroups and leads

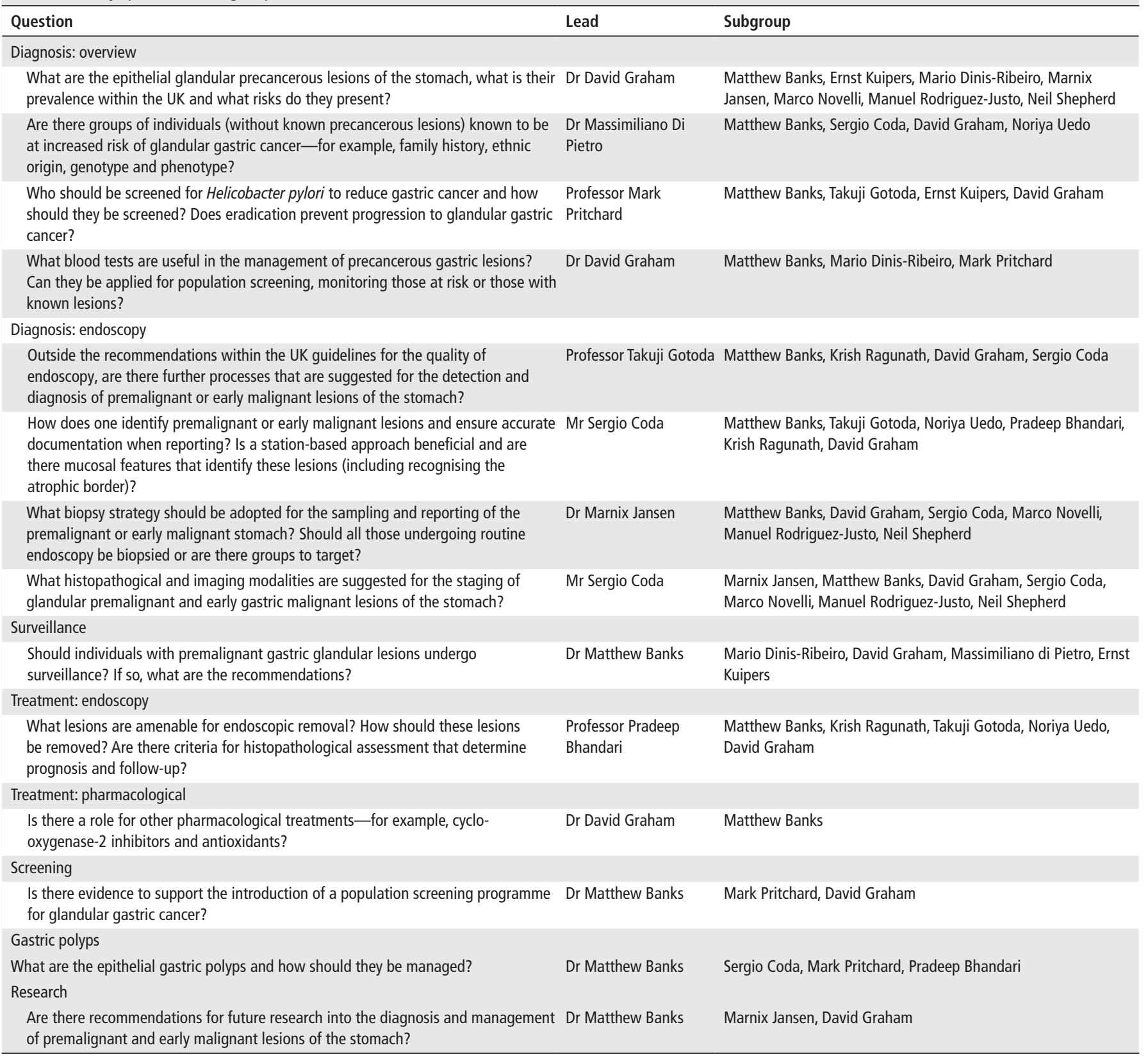

Each lead and subgroup formulated a set of clinical questions pertinent to each key question using the PICO (patients, interventions, controls and outcomes) system, within which these four critical components were predefined as precisely as possible. The importance of each PICO outcome was ranked numerically by the subgroups on a 1-9 scale (7-9: critical; 4-6: important; $1-3$ : of limited importance) according to the clinical importance of each. Those outcomes of $\leq 3$ were excluded.

\section{Literature searches, evidence synthesis and grading of evidence}

A literature search was undertaken by each subgroup with predefined search terms, electronic databases used (PubMed, Embase, Cochrane and Medline) and the time period covered. Where appropriate, conference abstracts were used to help formulate recommendations, provided that these were of sufficient scientific rigour.
The PICOs were used to guide the search for evidence and the highest quality studies were prioritised. Systematic reviews or meta-analyses were selected initially. Where systematic reviews, meta-analyses or critically appraised articles were unavailable, lower levels of evidence were selected. Where reviews use poorquality studies such as that by Spence et al, ${ }^{13}$ for example, where the review was based on small, often single-centre cohort studies resulting in high levels of heterogenicity, this is discussed in the evidence review and highlighted in the evidence tables. In this instance, more weight is given to larger studies using large databases, which are likely to be more accurate. This hierarchy of searching was performed in a pyramidal sequence from top (high quality) to bottom (low quality), cascading from recently published systematic reviews of randomised controlled trials (RCTs) or observational studies, to RCTs, and subsequently to observational studies providing that no evidence is available from the higher quality categories. The selected evidence was 
Table 2 Evidence level

\begin{tabular}{ll}
\hline Evidence level & \\
\hline High quality & $\begin{array}{l}\text { One or more well-designed and well-executed randomised controlled trials (RCTs) that yield consistent and directly applicable results } \\
\text { This level also means that further research is very unlikely to change our confidence in the estimate of effect }\end{array}$ \\
Moderate quality & $\begin{array}{l}\text { RCTs with important limitations (ie, biased assessment of the treatment effect, large loss to follow-up, lack of blinding, unexplained heterogeneity), indirect } \\
\text { evidence originating from similar (but not identical) populations of interest and RCTs with very small numbers of participants or observed events } \\
\text { In addition, evidence from well-designed controlled trials without randomisation, from well-designed cohort or case-controlled analytical studies, and from } \\
\text { multiple time series with or without intervention is included in this category } \\
\text { This level also means that further research will probably have an important impact on our confidence in the estimate of effect and may change the } \\
\text { estimate }\end{array}$ \\
$\begin{array}{l}\text { Observational studies would typically be rated as low quality because of the risk for bias* } \\
\text { Low quality } \\
\text { This level also means that further research is very likely to have an important impact on our confidence in the estimate of effect and will probably change } \\
\text { the estimate }\end{array}$ \\
$\begin{array}{l}\text { Evidence is conflicting, of poor quality or lacking, and hence the balance of benefits and harms cannot be determined } \\
\text { Any estimate of effect is very uncertain as evidence is either unavailable or does not permit a conclusion }\end{array}$
\end{tabular}

*Quality of evidence based on observational studies may be rated as moderate or even high, depending on circumstances under which evidence is obtained from observational studies. Factors that may contribute to upgrading the quality of evidence include a large magnitude of the observed effect, a dose-response association or the presence of an observed effect when all plausible confounders would decrease the observed effect.

tInsufficient evidence to determine for or against routinely providing a service.

tabulated in an evidence table categorising each study into the study design, intervention/clinical question, participants/population, reference standard, results and conclusions/comments.

Although randomised controlled studies are deemed the most appropriate type of study to assess the effectiveness of an intervention, other types of studies were included to assess types of effectiveness, such as ways of delivering service or outcomes from registries in clinical practice. If evidence from studies was weak or contradictory, searches for alternative sources were undertaken to see if the evidence concurred or contradicted (triangulation of searches). All available negative studies were included in the searches. The subgroups discussed the external validity of the studies and whether the study outcomes are applicable to the target population for the guidelines. Literature searches were transparent and reproducible to reduce 'dissemination biases'.

All evidence tables and references were downloaded to a shared reference manager (F1000Workspace), to which all GDG members had access. Shortly before the statements were finalised, a further literature search was undertaken. The quality of the evidence was assessed following the GRADE terminology using table $2 .{ }^{14}$ Evidence is graded as high, moderate, low and very low quality (table 2 ).

\section{Recommendations}

The body of evidence for many questions was of low quality. Thus many statements were based on the consensus of the GDG given the limitations of the evidence.

Recommendations were made by each subgroup pertaining to the PICOs and graded as above with the strength of each recommendation. Recommendation strength is based on four factors:

1. Balance between desirable and undesirable effects (not considering cost).

2. Quality of the evidence across critical/important outcomes.

3. Patients' values and preferences.

4. Costs (resource use).

There are two grades of recommendations: strong, where the benefits clearly outweigh the risks and burden ('We recommend'); and weak, where the benefits were closely balanced with risks/burden ('We suggest').

Once consensus was reached within the subgroup, the level of agreement for the recommendations was obtained from the whole GDG by anonymised voting. Level of agreement was subdivided into five categories: strongly agree, agree, undecided, disagree and strongly disagree. All comments were used to amend recommendations where appropriate, and a second round of voting was undertaken for any modifications. All results and comments were anonymously sent to the GDG Chairman (MB). Where the GDG level of agreement was consistently below $90 \%$ for strongly agree and agree after three rounds of voting, the recommendation was excluded.

\section{SUMMARY OF STATEMENTS, RECOMMENDATIONS AND SUGGESTIONS}

Pathogenesis, diagnosis and epidemiology of premalignant and early malignant gastric lesions

Pathogenesis and epidemiology of premalignant and early

malignant gastric lesions

What are the precancerous lesions of the stomach, what is their prevalence within the UK and what risk do they confer?

Patients with GA and GIM have an increased risk of gastric adenocarcinoma. Increased severity of atrophy and extent of intestinal metaplasia is associated with an increased risk of cancer (evidence level: moderate quality; level of agreement: 100\%).

Visible low-grade gastric dysplasia has an increased risk of prevalent HGD and gastric adenocarcinoma (evidence level: low quality; level of agreement: 100\%).

Non-visible, low-grade gastric dysplasia increases the risk of gastric adenocarcinoma, but the magnitude of this risk is unknown (evidence level: low quality; level of agreement: 93\%).

Gastric HGD carries a significant risk of progression to gastric adenocarcinoma (evidence level: moderate quality; level of agreement: 100\%).

\section{GA, GIM and dysplasia}

For the purposes of this guideline, CAG collectively includes GA and GIM. It is important to define the histopathology of the premalignant stomach in order to understand progression to cancer and its endoscopic appearances. The normal gastric mucosa is divided into two compartments, and includes the gastrin and mucus-secreting glands of the antrum and the acid and pepsinogen-secreting oxyntic glands of the corpus. GA is defined as the loss of pre-existent glands native to the gastric compartment. The two phenotypic features of GA include the loss of glandular mass with fibrosis of the lamina propria and 
replacement of the native gastric glands by metaplastic or pseudopyloric glands.

The Correa cascade describes the stepwise progression of precursor lesions towards intestinal-type gastric cancer. ${ }^{9} \mathrm{H}$. pylori infection initiates the cascade through non-atrophic chronic gastritis, GA, GIM and finally, dysplasia. ${ }^{15}$

\section{Gastric atrophy}

GA is diagnosed histopathologically by two specific features: the presence of chronic inflammatory cells, including lymphocytes and plasma cells that expand the lamina propria, and the loss of the pre-existent gastric glands.

There is inconsistency in the histopathological diagnosis and severity of GA between pathologists, resulting in low interobserver agreement by histopathologists when staging GA using the OLGA (Operative Link on Gastritis Assessment) staging system, based on biopsies taken using the Sydney protocol. ${ }^{16} 17$ Recent studies, however, have demonstrated that accurate endoscopic staging of the severity of GA are strongly linked to gastric adenocarcinoma risk and that the interobserver and intraobserver agreement of endoscopic severity assessment, in experienced hands, is moderate to excellent. ${ }^{16} 18$

The prevalence of CAG (including GA) worldwide correlates with the prevalence of $H$. pylori-associated gastritis, increases with age and tends to be slightly more common in men. Prevalence is typically determined using gastroscopy and serum pepsinogen. In Western populations, the prevalence varies from $0 \%$ to $8.3 \%$, depending on age. ${ }^{4}{ }^{19-21}$ Studies performed in high-incidence areas such as Japan and China showed a prevalence of CAG of between 33\% and 84\%. ${ }^{2022} 23$

Studies exploring the risk of progressing from CAG to gastric adenocarcinoma report a range of between $0 \%$ and $10 \%$, with an annual incidence (person-year) of $<1 \%$ (range $0-1.2 \%$ ). This is regardless of whether the study population is from a highrisk or low-risk area. ${ }^{1324}$ This is roughly comparable with other premalignant conditions of the digestive tract, such as Barrett's oesophagus and colonic adenomatous polyps, where there are established guidelines on surveillance. A Swedish observational, population-based cohort study, reviewing biopsy samples of 405172 patients from 1979 to 2011 , demonstrated that 1 in 50 patients with GA would develop gastric adenocarcinoma within 20 years (an annual risk of progression of $0.1 \%$ ). ${ }^{15} \mathrm{~A}$ second Dutch study exploring the follow-up data on 22365 patients diagnosed with CAG found the overall annual incidence for the development of gastric cancer in patients with GA was $0.1 \%{ }^{25}$ This increased to $0.25 \%$ for GIM, $0.6 \%$ for LGD and $6 \%$ for HGD within 5 years after diagnosis. A recent systematic review found that the annual incidence in most studies varied from $0.1 \%$ to $0.5 \%$, but a pooled analysis was not undertaken as there was significant heterogeneity between studies $\left(\mathrm{I}^{2}\right.$ statistic of $94 \%) .{ }^{13}$ This is partly explained by the poor quality of evidence in this systematic review derived from small cohorts of patients from single centres.

Using endoscopic grading, Japanese investigators found the cumulative 5-year incidence of gastric adenocarcinoma to be $0.7 \%$ in those with no or mild GA on endoscopic assessment, $1.9 \%$ with GA and $10 \%$ in severe endoscopic GA. ${ }^{26}$

\section{Gastric intestinal metaplasia}

GIM is a common finding in studies of patients undergoing diagnostic upper GI endoscopy-in particular, in those with a current or past $H$. pylori infection. GIM prevalence also increases with $H$. pylori infection, patient age, smokers and also with a first-degree relative with gastric cancer. The overall prevalence of GIM in those undergoing routine endoscopy varies from $13.8 \%$ to $19 \%$ in Europe. ${ }^{27}{ }^{28}$ It is important to point out that the European population is not uniformly representative of that in the UK. For example the population studied by Olmez et al is from a high prevalence area of Eastern Turkey. A Dutch study found GIM to be present in $25.3 \%$ of patients undergoing endoscopy for dyspepsia. ${ }^{29}$ The prevalence in those infected with $H$. pylori was $33.9 \%$ compared with $15.2 \%$ of those who were not infected. This study also noted that GIM was present in $55 \%$ of patients with gastric ulcer and $100 \%$ of patients with intestinal-type gastric adenocarcinoma. A multicentre European study found the prevalence of GIM to be $31.4 \%$ in patients infected with $H$. pylori. ${ }^{30}$ In high-incidence areas such as Japan and China, the prevalence of GIM in H. pylori-infected individuals was $37 \%$ and $29.3 \%$, respectively. ${ }^{31}$ GIM was found in only $2 \%$ of those not infected with $H$. pylori. ${ }^{15} 32$

The risk of gastric adenocarcinoma is increased in those found to have GIM. One in 39 patients with GIM develops gastric adenocarcinoma within 20 years, ${ }^{15}$ with similar rates found by De Vries et al with an annual incidence of cancer of $0.25 \%$ at 5 years. ${ }^{25}$ A meta-analysis carried out by Zullo et al in 2000 found that the risk of gastric adenocarcinoma progression in those with GIM ranged from $0 \%$ to $10 \%$, with this range thought to be due to differing study sample sizes and follow-up periods. ${ }^{32}$ Similarly, a systematic review by Spence et al found the annual incidence in most studies ranged from $0.15 \%$ to $0.4 \% .^{13}$

The risk of gastric adenocarcinoma varies with the type and extent of GIM. There are three histological types of GIM, with type I or 'intestinal' being termed 'complete IM' and types II and III or 'colonic' termed 'incomplete IM'. 'Incomplete IM' has been suggested to carry an increased cancer risk compared with 'complete IM'. ${ }^{32-34}$ A Portuguese study showed that 31\% and $6.9 \%$ of those with 'incomplete IM' developed LGD and HGD, respectively, compared with only $8 \%$ of those with 'complete IM', who developed LGD only. ${ }^{35}$ Therefore, histological subtyping may have a role in establishing gastric cancer risk, although it should be noted that only a minority of patients with invasive gastric cancer seem to have incomplete IM. Additionally, it should be noted that the traditional diagnosis of 'complete IM' or 'incomplete IM' is made using enzyme-histochemical staining methods that are highly dependent on the person evaluating them and thus are not reproducible. Therefore, the GDG did not consider histological subtyping for the guidelines.

The extent of the distribution of GIM appears to be of key importance. Four patterns of GIM distribution have been described. ${ }^{36}$ The first, 'focal' GIM, consists of scattered foci, mostly in the lesser curvature and incisura. The second, 'antrum-predominant' GIM, involves most of the antrum and incisura angularis. These two patterns with less extensive involvement of the gastric mucosa consist almost exclusively of complete type IM. The third, 'magenstrasse' GIM spreads throughout the lesser curvature from the cardia to the pylorus, also involving the greater curvature of the prepyloric antrum. The fourth, 'diffuse' GIM involves the entire gastric mucosa, with the exception of the fundic areas. These more extensive types had a greater predominance of 'incomplete' GIM.

Several studies have demonstrated that more extensive GIM correlates with increased gastric adenocarcinoma risk. ${ }^{32} 36-38$ Of note, a Columbian study found that in comparison with focal or antral-predominant GIM, those with magenstrasse GIM had a 5.7-fold increased risk of gastric adenocarcinoma, while those with a diffuse pattern (antrum and gastric body) had a 12.2fold increased risk. Alongside this, an Italian study showed that 
a $>20 \%$ extension of GIM identified those at increased risk. ${ }^{32} \mathrm{~A}$ Japanese study found the cumulative 5-year incidence of gastric adenocarcinoma to be $1.5 \%$ in those without GIM, compared with $5.3 \%$ in those with GIM in the antrum only and $9.8 \%$ in those with GIM in the antrum and corpus. ${ }^{26}$

Unlike the OLGA staging system, the OLGIM (Operative Link on Gastric Intestinal Metaplasia) staging system, using the presence of GIM, has proved to be a reproducible marker of risk with high interobserver agreement and strong association with OLGA stage. ${ }^{39} 40$ The GDG did not agree that OLGIM should be routinely used in clinical practice, although it has practical applicability in research.

Intestinal metaplasia of the gastric cardia has been reported to vary from $5 \%$ to $25 \%$ in those having endoscopy ${ }^{41} 42$ and may confer an increased risk of dysplasia and cancer, although the incidence is not clear. Sharma and colleagues found 1 of 76 patients with prevalent LGD in cardia intestinal metaplasia, defined as that just below the gastro-oesophageal junction, compared with 20 of 177 having low-grade and high-grade dysplasia with short segment Barrett's oesophagus $(<3 \mathrm{~cm}) .{ }^{43}$

\section{Gastric dysplasia}

The endoscopic prevalence of gastric dysplasia varies from $0.5 \%$ to $3.7 \%$ in Western countries and from $9 \%$ to $20 \%$ in areas with a high incidence of gastric adenocarcinoma. ${ }^{44-47}$ The identification of gastric dysplasia should also alert the endoscopist to the possibility of synchronous gastric cancer. Studies have demonstrated the incidence of synchronous gastric adenocarcinoma in those with gastric dysplasia to be up to up to $30 \% .^{44}$

A review of the natural history of gastric dysplasia showed that patients with HGD had a rate of malignant progression or synchronous malignant lesions of 60-85\% over a median interval period of 4-48 months. ${ }^{44}$ Song et al, in their observational study, found that 1 in 19 patients with dysplasia progressed to gastric adenocarcinoma within 20 years, although no differentiation was made between those with LGD or HGD. ${ }^{15}$ de Vries et al noted the annual incidence of gastric cancer was $6 \%$ in patients with HGD within 5 years. ${ }^{25}$ Of note, it is difficult on histopathology to distinguish between HGD and gastric adenocarcinoma from small biopsy samples.

The risk of progression in individuals with LGD is less clear. There is evidence to show that LGD will regress in $38-75 \%$ of patients and persist in 19-50\%. In the LGD lesions that persist, the risk of malignant progression ranges from $0 \%$ to $23 \%$ in the published literature over 10-48 months. ${ }^{44}$ de Vries et al reported that the annual incidence of gastric adenocarcinoma in those with LGD was $0.6 \%$ within 5 years after diagnosis. ${ }^{25}$

Visible LGD following resection is upstaged in 25-35\% of lesions, including those of $<1 \mathrm{~cm}$, with an adenocarcinoma rate of $6.9 \%{ }^{44}$ We have therefore suggested that the risk of prevalent HGD or gastric adenocarcinoma is greater in visible LGD. Although there is uncertainty about the natural history of non-visible LGD, the evidence suggests that there is an increased rate of progression, but the magnitude is unclear.

A summary of the risks of gastric cancer is shown in table 3 .

\section{Risk factors for gastric adenocarcinoma}

Are there groups of individuals (without known precancerous lesions) known to be at increased risk of gastric adenocarcinoma?

There are subgroups of individuals (other than those with $H$. pylori infection) who have an increased risk of gastric adenocarcinoma. The strongest risk factors include family history of gastric adenocarcinoma and serologically confirmed pernicious anaemia
Table 3 The risk of cancer for patients with gastric atrophy and intestinal metaplasia

\begin{tabular}{lll}
\hline & $\begin{array}{l}\text { 5-Year incidence of } \\
\text { gastric cancer (\%) }\end{array}$ & Annual incidence (\%) \\
\hline All GA & 1.9 & $0.1-0.5$ \\
\hline Mild GA & 0.7 & \\
Severe GA & 10 & $0.15-0.4$ \\
All GIM & & 0.25 \\
& & \\
Antral GIM & 5.3 & \\
Antral and corpus GIM & 9.8 & \\
& Interval of 4-48 months & 0.6 \\
\hline Low-grade dysplasia & $0-23$ & 6 \\
\hline High-grade dysplasia & $60-85$ & \\
\hline
\end{tabular}

GA, gastric atrophy; GIM, gastric intestinal metaplasia.

with GA. Additional risk factors include non-white origin, history of gastric surgery for benign disease longer than 15 years, age older than 45 years, male sex, high salt intake and smoking (evidence level: low quality; level of agreement: 93\%).

Several factors increase the risk of developing malignant lesions of the stomach, which should be taken into account when considering screening on an individual basis. These risk factors might help in the development of larger scale screening programmes in the future and relate to multiple subcategories including patient demographics and epidemiological factors, as well as personal, medical, social and family history. Unfortunately, the grade of evidence on the link between risk factors and gastric cancer development is low, as this comes almost universally from retrospective or observational studies, including meta-analyses from these studies. The working group has analysed the evidence related to 11 risk factors based on the available literature.

Family history appears to be one of the strongest risk factors. Three case-control studies (from Japan, Poland and South Korea) for a total of 1024 gastric cancer cases showed that the OR for gastric adenocarcinoma in first-degree relatives of patients with gastric adenocarcinoma ranged from 2.3 to $3.5 .{ }^{47-50}$ In another US population case-control study, the risk of gastric adenocarcinoma was higher in subjects reporting two or more family members with gastric adenocarcinoma $(\mathrm{OR}=5-12.1$, 95\% CI 1.35 to 108.5$)$. Two additional studies have suggested that the risk is higher and the age of onset lower in those with a family history of early-onset ( $<50$ years) gastric adenocarcinoma. ${ }^{51} 52$ In addition, a retrospective cohort study on US veterans with GIM indicated that a family history of gastric adenocarcinoma was associated with a HR of 3.8 (95\% CI 1.5 to 9.7) for gastric adenocarcinoma development. ${ }^{53}$ In keeping with this evidence, monozygotic and dizygotic twins of individuals with gastric adenocarcinoma have 9.9-fold and 6.6-fold higher risk of gastric adenocarcinoma, respectively, compared with twins of subjects without gastric adenocarcinoma. ${ }^{54}$ It is important to consider that familial aggregation occurs in approximately $10 \%$ of cases, which in low-incidence countries of gastric adenocarcinoma probably relates to heritable pathogenic mutations. However, truly hereditary cases are deemed to account for approximately $1-3 \%$ of all gastric adenocarcinoma cases and include three main subgroups: hereditary diffuse gastric cancer (HDGC), familial intestinal gastric cancer and other single-gene syndromes associated with a possible increased risk of gastric adenocarcinoma. ${ }^{55}$ These single-gene syndromes include gastric adenocarcinoma and proximal polyposis of the stomach, ${ }^{565} \mathrm{Li}$-Fraumeni syndrome, 
Box 1 Criteria for referring to genetic services

\section{Suspected familial gastric cancer}

- Gastric cancer in one family member before age 40 .

- Or gastric cancer in two first-degree/second-degree relatives with one diagnosis before age $50^{*}$.

- Or gastric cancer in three first-degree/second-degree relatives independent of age*.

Suspected hereditary diffuse gastric cancer

- One case of diffuse gastric cancer before age 40 .

- Or two cases of gastric cancer regardless of age in two first-degree/second-degree relatives, at least one confirmed diffuse gastric cancer*.

- Or personal and family history of diffuse gastric and lobular breast cancers, with one diagnosis before age 50 .

- A personal or family history of cleft lip/palate in a patient with diffuse gastric cancer.

- In situ signet ring cells or pagetoid spread on gastric biopsies.

* In order to account for significant family history, the affected relatives need to be within the same side of the family (maternal or paternal).

familial adenomatous polyposis (FAP), Peutz-Jeghers syndrome, juvenile polyposis, Cowden's syndrome/PTEN hamartoma tumour syndrome (PTHS) and Lynch syndrome. ${ }^{58}$ More than $90 \%$ of individuals with a phosphatase and tensin homolog (PTEN) pathogenic variant who underwent at least one upper or lower endoscopy were found to have polyps. ${ }^{59}$ Histological findings varied, ranging from ganglioneuromatous polyps, hamartomatous polyps and juvenile polyps, to adenomatous polyps. The association of gastric cancer with FAP is mainly based on case reports and thus remains weak.

Although this guideline does not cover management of hereditary gastric cancer, it is important to know how to take an appropriate family history, in order to suspect familial gastric cancer and know when to refer for genetic counselling. Outside the specialised genetic counselling clinic, where the three-generation comprehensive pedigree is recommended, in standard clinical practice it is recognised that it is sufficient to focus on first-degree (parents, children and siblings) and second-degree relatives (grandparents, aunts/uncles, nieces/nephews, grandchildren and half siblings) in order to assess the empirical individual risk profile. ${ }^{60}$ Affected relatives should be from the same lineage (maternal or paternal) and be first-degree relatives of each other. The age of diagnosis, the histological type of gastric cancer (if possible) and the occurrence of GI polyps and other cancers, including colorectal, ovarian, breast and pancreatic cancer, should be recorded. Criteria for referring to genetic services are outlined in box $1 .^{6162} \mathrm{It}$ is important to note that the criteria for a clinical diagnosis of HDGC have been slightly relaxed in the more recent updated clinical guidelines. A history of breast cancer is particularly important for a clinical suspicion of familial gastric cancer as there is clustering between gastric and breast cancers in HDGC (lobular type) and in other familial cancer syndromes, including Peutz-Jeghers and Cowden. ${ }^{61} 63$ Individuals who fulfil the family history criteria for suspected familial gastric cancer in the absence of an identified mutation could be considered for endoscopic surveillance. Ideally, this should be offered as part of a clinical trial as there are no validated surveillance protocols in this group of individuals. In the absence of evidence, we would suggest 3-yearly endoscopy with high-definition endoscopy and image enhancement as a possible protocol. Details of genetic centres in the UK can be found on the British Society for Genetic Medicine website (https://www. bsgm.org.uk).

Many genetic polymorphisms have been shown to be associated with an increased risk of gastric cancer, in particular related to inflammatory response genes, such as interleukins, interleukin receptors and tumour necrosis factor. ${ }^{64}$ There is insufficient evidence to recommend routine DNA genotyping to inform clinical decisions outside cases that meet the criteria for familial gastric cancer. ${ }^{55}$ A detailed discussion of this topic is beyond the scope of this guideline.

Pernicious anaemia is another strong risk factor for gastric adenocarcinoma development. A systematic review by Vannella and coworkers found that the pooled incidence of gastric adenocarcinoma in patients with pernicious anaemia is $0.27 \%$ a year with a relative risk (RR) of $6.8(95 \%$ CI 2.6 to 18.1$) .{ }^{65}$ A more recent population-based case-control study on 1138390 cancer cases compared with 100000 matched controls found that in patients with pernicious anaemia, the ORs for gastric adenocarcinoma and gastric neuroendocrine tumours were 2.18 (95\% CI 1.94 to 2.45 ) and 11.4 (95\% CI 8.9 to 14.7$)$, respectively. ${ }^{66}$ Since these were unverified cases of pernicious anaemia, this was probably a marked underestimate of the cancer risk in patients with definite pernicious anaemia. Substantiating a diagnosis of pernicious anaemia, however, can be challenging. Megaloblastic anaemia and vitamin $\mathrm{B}_{12}$ deficiency can depend on a multitude of clinical conditions, and the diagnostic accuracy of autoantibodies is low (low specificity for antiparietal cell antibodies and low sensitivity for anti-intrinsic factor antibodies). ${ }^{67} 68$ Therefore, in order to stratify cancer risk, it is essential that histopathological evidence of autoimmune atrophic gastritis corroborates the serological diagnosis.

It has long been suggested that a history of gastric surgery for benign disease is a risk factor for gastric adenocarcinoma. Three early retrospective cohort studies have shown an increased gastric adenocarcinoma risk in individuals carrying a gastric stump from previous surgery for non-malignant disease compared with the general population, ${ }^{69-71}$ while one case-control study did not reach similar conclusions. ${ }^{72}$ The discrepancy may be resolved by a more recent and large retrospective study, which found that the risk of gastric adenocarcinoma in a gastric stump only increases after 30 years from gastric surgery with a standardised incidence ratio of 2.29 (95\% CI 1.38 to 3.57$){ }^{73}$ Hence, it is reasonable to conclude that the risk of gastric adenocarcinoma arising in the gastric stump is unlikely to be significantly increased within the first 15 years after surgery. However, it should be noted that these data may be confounded by $H$. pylori status.

Ethnicity is strongly related to the risk of gastric adenocarcinoma. Several retrospective cohort studies conducted in US and UK populations showed that white subjects appear to have a lower risk than Asian subjects (2.1-fold higher incidence), black subjects (1.7-fold higher incidence) and Hispanic-Latino subjects (1.7-fold higher incidence) ${ }^{74-78}$ Among Asian people, Chinese and Korean subjects appear to have the highest risk of a premalignant stomach with an OR of $4.77(95 \%$ CI 4.54 to 5.01 ) and 7.39 (95\% CI 7.06 to 7.73 ), respectively. ${ }^{21}$ These differences are likely to be, at least in part, explained by differences in $H$. pylori prevalence, $H$. pylori subtypes and host genotypes.

There is evidence that older age is a risk factor for developing gastric adenocarcinoma, reflecting the duration of $H$. pylori gastritis. Different studies report different age groups to study this association-for example, 45, 50 or 75 years of age; hence, it is difficult to identify an age cut-off point to guide clinical 
decisions. ${ }^{25} 7980$ However, three studies have shown that age $>45$ years is associated with a significant OR between 1.92 and 3.1 for progression of premalignant stomach to gastric adenocarcinoma. ${ }^{258182}$ Hence, it is reasonable to consider 45 years of age as a useful threshold for potential screening strategies.

Men appear to be at increased risk of gastric adenocarcinoma compared with women. Two cohort studies and one casecontrol study showed different strengths of this association, with a relative incidence in men compared with women, ranging from 1.3-fold to 3 -fold higher. ${ }^{74} 79$ This does not apply to cases of gastric adenocarcinoma occurring before the age of 40 years, which are probably due to the domineering genetic background responsible for early-onset cancer. ${ }^{5583}$

Smoking seems to be an additional risk factor for development of gastric adenocarcinoma, particularly, but not limited to, gastric cardia adenocarcinoma. Smoking appears to be responsible for $18 \%$ of gastric adenocarcinoma risk, ${ }^{84}$ and this relates to an increase in the risk by 1.45 -fold to 2 -fold according to different studies. ${ }^{85} 86$ However, one meta-analysis showed a trend for increased risk of gastric adenocarcinoma development only in current smokers, which did not meet statistical significance $(\mathrm{OR}=1.49 ; 95 \% \mathrm{CI} 0.99$ to 2.225$) .{ }^{87}$

Other risk factors that have been studied and were assessed by the working group include alcohol consumption, diabetes, obesity and use of protein pump inhibitors. ${ }^{88-90}$ For these factors the available evidence is conflicting or does not show a clear relation to the risk of gastric adenocarcinoma development. In particular for obesity there is sufficient evidence to conclude that this is associated with the risk of cardia cancer, but not non-cardia gastric cancer. ${ }^{91}$ A number of recent studies have investigated the association between PPIs and gastric cancer. A study by Cheung et al assessing patients who were treated for $H$. pylori demonstrated an association between PPI use and gastric cancer in a population from Hong Kong. PPI use was associated with a 2.44-fold increase in cancer. No increase was detected in $\mathrm{H} 2$ antagonists, and most cancers were non-cardia. There were significant flaws in this study. Most importantly, the PPI group were 10 years older on average than the non-PPI group, and as gastric cancer increases with age it would be expected that the risk would be greater in this group. A Swedish study showed a similar increase in gastric cancer in PPI users, with the greatest risk in younger men. No difference was found, however, in a US study looking at pantoprazole. It can be concluded that although there is an association between PPI use and gastric cancer, no causal link has been proved. Furthermore, this association may be limited to specific populations and limited to patients who have had H. pylori eradication. ${ }^{92-94}$ We make reference to a BSG position statement, ${ }^{95}$ which recommends that if acid suppression is required after $\mathrm{H}$. pylori eradication, $\mathrm{H} 2$ antagonists should be used first, and if these are ineffective PPIs can be initiated with the above information being shared with the patient. In other patients there is no evidence to advise against the use of PPIs first line for short-term use. Prolonged use should be accompanied by an explanation of the evidence for possible risks as described above.

\section{H. pylori and gastric cancer}

Who should be tested and treated for $\mathrm{H}$. pylori to reduce the risk of gastric cancer?

We do not recommend testing and treating for $H$. pylori to reduce the incidence of gastric cancer in low-incidence areas such as the UK (evidence level: high quality; grade of recommendation: high; level of agreement: 100\%).
Several studies in rodents support the hypothesis that eradication of $H$. pylori infection prevents the subsequent development of gastric adenocarcinoma. ${ }^{9697}$ However, the benefits were reduced in these animal models when infection was eradicated at later stages in the natural history of the disease. Multiple human cohort studies and randomised clinical trials have now been performed to assess whether testing for H. pylori in the asymptomatic population and its subsequent eradication in infected subjects reduces the subsequent incidence of gastric cancer. In turn, these studies have been subjected to systematic reviews and meta-analyses by at least three independent research groups. It was concluded that searching for and eradicating $H$. pylori reduced the incidence of gastric adenocarcinoma in healthy asymptomatic infected individuals, with the number of patients needed to treat as low as 15 for Chinese men, but as high as 245 for US women. ${ }^{98-100}$ The benefits of eradication varied with baseline gastric cancer incidence, but did not differ with study design, sex or follow-up period baseline risk. ${ }^{101}$

Thus, there is compelling evidence that $H$. pylori eradication is effective at reducing the subsequent risk of gastric adenocarcinoma development. In areas of low to moderate gastric adenocarcinoma incidence such as the UK, however, it has been suggested that this is not a cost-effective strategy. However, as recently argued by O'Connor et al, ${ }^{102}$ there may be additional benefits associated with $H$. pylori eradication, such as a reduction in peptic ulcer disease and dyspepsia, and these have not been considered in all the proposed economic models. Other potential consequences of widespread $H$. pylori eradication therapy should also be considered when considering the risk:benefit ratio of such a strategy. These include the potential emergence of antibiotic-resistant strains of the organism and treatment-associated side effects, some of which, although rare, can be serious, such as Clostridium difficile infection. ${ }^{102}$

Therefore, although $H$. pylori eradication is a feasible, efficacious and potentially cost-effective method (at least in some populations) for reducing the risk of subsequent gastric adenocarcinoma development, its use has not yet been adopted anywhere in the world. The final results of a very large Chinese trial involving more than 180000 participants will be available within the next few years, and its results may influence future public health policy in this area, particularly in East Asian countries. ${ }^{103}$ However, at present, in areas of relatively low gastric cancer incidence such as the UK, we cannot currently recommend population testing and treating for $H$. pylori as a means for preventing subsequent gastric cancer development

Does eradication of $\mathrm{H}$. pylori prevent progression to cancer?

We recommend $H$. pylori eradication to reduce the risk of gastric adenocarcinoma development in patients who have H. pylori-associated GA (evidence level: high quality; grade of recommendation: high; level of agreement: 100\%).

We suggest that $H$. pylori eradication may be of some benefit to reduce the risk of developing gastric adenocarcinoma in those who already have H. pylori-associated GIM, dysplasia or cancer (evidence level: high quality; grade of recommendation: weak; level of agreement: 100\%).

Although there is good evidence that $H$. pylori eradication reduces the subsequent risk of gastric adenocarcinoma development, it has been hypothesised that there may be a 'point of no return' in the precancerous cascade. This question has been assessed in many studies and has been the topic of two recent independent systematic reviews and meta-analyses and most recently a large RCT.

Chen et al performed a meta-analysis of 10 studies from eight RCTs with a total of 7955 participants. ${ }^{104}$ These authors 
divided participants into subgroups based on their baseline diagnoses, comparing a group without GIM (normal, non-atrophic gastritis, GA) and a group with GIM (GIM or dysplasia). $H$. pylori treatment compared with controls significantly reduced the risk of gastric cancer, with a pooled RR of 0.64 (95\% CI 0.48 to 0.85 ). Subgroup analysis for patients with non-atrophic gastritis and GA yielded similar results $(\mathrm{RR}=0.25,95 \% \mathrm{CI} 0.08$ to 0.81 ). However, this difference was not seen in patients with GIM or dysplasia ( $\mathrm{RR}=0.88,95 \% \mathrm{CI} 0.59$ to 1.31 ). The authors concluded that $H$. pylori treatment no longer reduced the risk of gastric adenocarcinoma development once patients had developed GIM or dysplasia.

Rokkas et $a l^{105}$ recently published a second independent meta-analysis on this topic. ${ }^{105}$ They analysed 26 studies (10 RCTs and 16 cohort studies) and included 52363 subjects. The risk of gastric adenocarcinoma among patients in whom $H$. pylori had been successfully eradicated was significantly lower than that among controls (pooled RRs (95\% CI) 0.56 (0.48 to 0.66 ), $\mathrm{Z}=-7.27, \mathrm{p}=0.00001)$. In patients with precancerous lesions, however, subgroup analyses showed that patients with non-atrophic or GA benefited from $H$. pylori eradication to decrease the risk of gastric cancer development, whereas those with GIM or dysplasia did not. In contrast to these data, a recent large, randomised, placebo-controlled trial demonstrated a significant reduction in metachronous gastric adenocarcinoma rates after endoscopic resection of intramucosal cancer and HGD in the $H$ pylori eradication group (13.4\% vs $7.9 \%$; $\mathrm{p}=0.03) .{ }^{106}$ Furthermore, there appeared to be a reversal in the severity of both GA and GIM in the corpus, but not in the antrum.

Current high-quality data therefore suggest that while eradication of $H$. pylori reduces subsequent gastric adenocarcinoma risk in patients who have non-atrophic or GA, these benefits are not consistently maintained in patients who have developed GIM, dysplasia or cancer. The published meta-analyses did not, however, examine whether the histological type, severity and location of GIM or dysplasia influence this response to treatment. Although early data do not consistently support the use of $H$. pylori eradication in patients who already have established intestinal metaplasia, dysplasia or cancer, the aforementioned RCT provides evidence to treat those with established cancer or HGD.

Although most of the published evidence is from the Far East, there is no compelling evidence to suggest that the fundamental process of gastric carcinogenesis differs according to geographical location. Published evidence suggests that $H$. pylori eradication appears to prevent gastric cancer development in some patients who have atrophic gastritis. Despite the lack of studies from the UK and Europe, $H$. pylori eradication is likely to benefit some European patients who have atrophic gastritis, and has a very low risk of causing serious adverse events. Further studies in European populations are needed to expand the pool of evidence that will influence this recommendation when these guidelines are revised.

The success of an $H$. pylori eradication strategy to try to prevent cancer development in patients who have premalignant gastric conditions will obviously be influenced by the success rate of the bacterial eradication regimen that is used. The choice of eradication regimen is beyond the scope of these guidelines, but its efficacy should be monitored. Regimens other than the classic triple therapy may need to be employed, particularly in geographical locations where the rates of $\mathrm{H}$. pylori antibiotic resistance are known to be high.

In addition to the conventional Correa cascade of gastric carcinogenesis, there are other gastric conditions that are potentially associated with an increased risk of gastric cancer development-in particular, some gastric polyps. ${ }^{107}$ Although there is good evidence to suggest that $H$. pylori eradication leads to the regression of gastric hyperplastic polyps, no studies appear to have analysed whether this influences the subsequent risk of gastric adenocarcinoma. ${ }^{108-110}$ Studies on the effects of $H$. pylori eradication in patients with gastric adenomas are too small to draw any valid conclusions. ${ }^{111}$

\section{Biomarkers and gastric cancer}

What biomarkers are useful in the management of these lesions?

Can they be applied to population screening, monitoring those at risk or those with known lesions?

Measurement of serum pepsinogen I and serum pepsinogen I/ II ratio alone or in combination with $H$. pylori serology, and/ or gastrin-17 can identify individuals with extensive atrophic gastritis (evidence level: low quality; grade of recommendation: weak; level of agreement: 93\%).

We do not recommend the use of biomarkers as a screening tool in areas with a low incidence of gastric adenocarcinoma, such as the UK (evidence level: low quality; grade of recommendation: weak; level of agreement: 100\%).

\section{CAG, dysplasia and gastric adenocarcinoma}

Pepsinogen I (PGI) is mainly secreted by chief and mucous neck cells in the fundic mucosa, while pepsinogen II (PGII) is also secreted by pyloric and duodenal Brunner's glands. Approximately $1 \%$ of pepsinogens are found in the serum, with their serum level accepted as a marker for the morphological and functional status of the gastric mucosa. ${ }^{10} 112113$ Serum PGI and PGII levels both increase in gastric mucosal inflammation; however, as GA develops and specialised cells are lost, PGI and PGII levels decrease, usually more marked in PGI, resulting in low serum PGI and a low PGI/II ratio. ${ }^{114}$

The combined use of serum PGI and PGI/II ratio measurements is an accepted useful biomarker for premalignant and malignant gastric lesions. ${ }^{12}$ Studies exploring their use as a population screening tool, where those with a positive pepsinogen result progress to endoscopic examination, have been shown to be acceptable in screening asymptomatic populations, with a good uptake of invitations for endoscopy—over 60\%, in those with a positive pepsinogen result. ${ }^{115} 116$

\section{Biomarker detection of CAG, dysplasia and gastric cancer}

Numerous studies across many different countries and populations have explored the use of serum pepsinogen testing for detection of CAG, dysplasia and gastric adenocarcinoma. The majority of these studies are from countries with a higher incidence of these lesions than the UK. For countries with an incidence of gastric adenocarcinoma similar to that of the UK, a study by Broutet et al in 2003 assessed serum pepsinogen testing across 14 European nations and determined that the PGI/II ratio may be of use as a screening test. ${ }^{112} 117118$ However, in the majority of studies, the values used to define a positive pepsinogen test result and the study outcomes are reflective of populations that differ from the UK population. Additionally, many of these studies use differing cut-off values, which makes comparison difficult. However, the most frequently used values for these studies are a PGI $<70 \mathrm{ng} / \mathrm{mL}$ and a PGI/II ratio $\leq 3 .{ }^{112}$ Values can be affected by laboratory methodologies and population settings, and therefore may require adjustment if applied to the UK population. 
A meta-analysis by Huang et al found that serum pepsinogen testing had a sensitivity and specificity of $69 \%$ and $73 \%$ for gastric cancer diagnosis and 69\% and 88\% for CAG diagnosis, respectively. ${ }^{112}$ This analysis included a study population of over 30000 individuals, across 13 different countries and diagnosis confirmed with gastroscopy and biopsy. This is comparable with the 2004 meta-analysis performed by Dinis-Ribeiro et al, using a cut-off value of PGI $<50$ and PGI/II ratio $\leq 3$ for dysplasia detection, where sensitivity and specificity were $65 \%$ and $74-85 \%$, respectively. ${ }^{117}$ This included a study population of approximately 300000 patients. Finally, in a 2014 meta-analysis by Terasawa $e t$ al, a study population of approximately 32000 patients, in which individuals were prospectively followed up for between 3.9 and 14 years found that a positive pepsinogen test had a sensitivity of $57 \%$ and specificity of $76 \%$ for the development of gastric adenocarcinoma. ${ }^{119}$

\section{Biomarker population screening}

Although pepsinogen testing has been demonstrated as a useful tool for population screening in high-risk areas, its use in low-risk areas such as the UK has not been explored. ${ }^{116119120}$ In particular, one must consider whether the moderately effective sensitivities and specificities of pepsinogen testing are cost-effective for screening an asymptomatic population where the incidence of the disease is low. A 2015 study by Yeh et al used a mathematical simulation model to calculate the cost-effectiveness of population screening strategies based on biomarker and endoscopic technologies in the low-risk US population (defined as an age-standardised rate (ASR) $<10$ per 100 000). ${ }^{113}$ This study found that although one-time serum pepsinogen testing at the age of 50 could prevent one in four cases of gastric adenocarcinoma among men, it was not of high value in improving cancer outcomes. However, targeting the high-risk group of male smokers over 50 years old could be a cost-effective way to reduce mortality from gastric adenocarcinoma. ${ }^{113}$ This screening model requires further exploration.

H. pylori serology has been studied extensively both as a population screening tool alone, as part of a 'test and treat' strategy and in conjunction with pepsinogen testing. While its use as a screening tool or in the 'test and treat' strategy may have advantages in high-incidence areas (defined as an ASR $>20$ per 100000 ), the disadvantages, including low specificity and antibiotic resistance in low-risk populations, render its use redundant. ${ }^{121}$ The use of $H$. pylori serology in combination with pepsinogen is more accurate. In particular, the finding of negative serum anti- $H$. pylori IgG antibody and positive pepsinogen measurements suggests extensive GA, and thus these individuals are at highest risk of progression to cancer. ${ }^{10} 121$ The ABCD method for the detection of individuals with high gastric adenocarcinoma risk has been extensively investigated in high-risk populations. This method categorises patients tested for $H$. pylori serology (HP) and the ratios of serum PGI and PGII (sPG) into low risk (A: $\left.\mathrm{HP}-, \mathrm{sPG}^{-}\right)$, moderate risk (B: $\mathrm{HP}+$ and $\mathrm{sPG}-$ ) and high risk (C: $\mathrm{HP}+$ and $\mathrm{sPG}+$; $\mathrm{D}: \mathrm{HP}-$, sPG+). Although in a 20-year prospective study the HRs for developing gastric adenocarcinoma were 15 for group D when compared with group $\mathrm{A},{ }^{122}$ further evidence is required to support the use of these approaches in a low-risk population.

GastroPanel combines PGI, PGII, gastrin-17 and $H$. pylori serology. A recent meta-analysis assessed the performance of this serum panel test for the diagnosis of CAG in 4241 subjects. The sensitivity for CAG was $74.7 \%$ (95\% CI $62.0 \%$ to $84.3 \%$ ) and the specificity was $95.6 \%$ (95\% CI $92.6 \%$ to $97.4 \%)$. With a prevalence of CAG of $27 \%$ (median prevalence across the studies), the negative predictive value was $91 \%$ and the positive predictive value was $86 \% .{ }^{123}$ Thus, although studies to date have shown promise, there is little evidence to support its use, with conflicting data on its efficacy. ${ }^{124-126}$

Finally, note is made of studies exploring the detection of volatile organic compounds in exhaled breath that are associated with the detection of gastric adenocarcinoma. ${ }^{127}$

\section{Endoscopic diagnosis of premalignant or early malignant lesions of the stomach}

Ensuring high-quality endoscopic evaluation

Outside the recommendations within the UK guidelines for quality endoscopy, are there further processes that are suggested for the detection and diagnosis of premalignant or early malignant lesions of the stomach? Is a station-based approach beneficial?

We recommend that patients at higher risk for gastric adenocarcinoma, including GA and GIM, should undergo a full systematic endoscopy protocol of the stomach with clear photographic documentation of gastric regions and pathology. We suggest a minimum examination time of $7 \mathrm{~min}$ (evidence level: moderate quality; grade of recommendation: strong; level of agreement: $100 \%)$.

Endoscopy needs to be of high quality in order to detect dysplasia and early cancers, particularly in light of postendoscopy gastric cancer rates of $11.3 \% .{ }^{128}$ Recognising and targeting high-risk patients with GA and GIM may be the most effective means to improve gastric cancer detection, and possibly survival, in the UK. There are three basic principles, which are part of routine practice: cleaning of the gastric mucosa, adequate distention of the gastric wall by air insufflation and mapping the entire stomach.

Although there is little available evidence on the use of smooth muscle relaxants and mucosal cleaning techniques, the new BSG position statement on quality standards in upper gastrointestinal endoscopy recommends 'Adequate mucosal visualisation should be achieved by a combination of adequate air insufflation, aspiration and the use of mucosal cleansing techniques'. ${ }^{129}$ We recommend that this guidance is also applied to inspection of GA and GIM.

There is also a paucity of data on the assessment of a complete endoscopic procedure. In particular, no clinical trials have directly clarified key performance indicators or quality assurance (QA) for improving gastric cancer detection, and no studies have explored the outcomes of systematic screening protocols for the stomach. The European Society of Gastrointestinal Endoscopy (ESGE) performance measures for upper GI endoscopy have suggested that the inspection of the oesophagus, stomach and duodenum should last at least $7 \mathrm{~min}$ from intubation to extubation. ${ }^{130}$ This statement was based on a retrospective cohort study by Teh et al, which aimed to determine the diagnostic yield for early neoplastic lesions in the stomach. ${ }^{131}$ After evaluating 837 endoscopies of symptomatic patients with no history of gastric cancer, they found that a 'slow' endoscopist ( $>7$ min examination) was twice as likely to detect high-risk gastric lesions, defined as biopsy evidence of GA, GIM, gastric dysplasia or cancer, and three times as likely to detect a case of dysplasia or cancer compared with a 'fast' endoscopist $(<7 \mathrm{~min}$ examination). ${ }^{131132}$

Photographic documentation might be an indirect quality indicator. Endoscopists with longer procedure times, who take more than four pictures, detect more pathology. ${ }^{132}$ The ESGE 


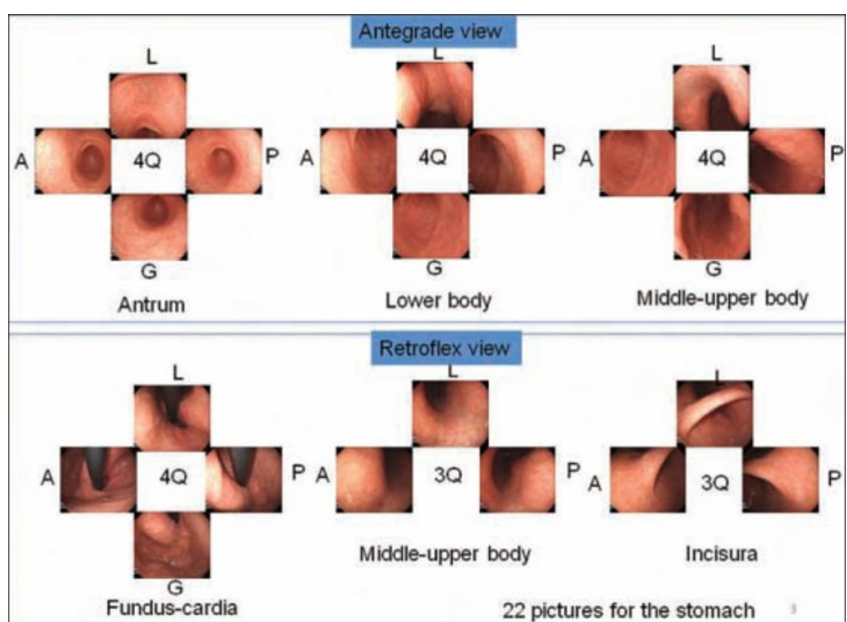

Figure 2 Systematic screening protocol for the stomach. This is a station-based approach whereby each area of the stomach is viewed and photographed in either a clockwise or counterclockwise manner. The 22 pictures are arranged according to the order of the procedure. $Q$, quadrant; $L$, lesser curvature; $A$, anterior wall; $G$, greater curvature; $P$, posterior wall; SSS, systematic screening protocol for the somach.

has recommended five areas in the stomach should be photographically documented, including the cardia and fundus in inversion, corpus in forward view including lesser curvature, corpus in retroflex view including greater curvature, angulus in partial inversion, and antrum. Images may be used in case discussions, patient management and compared with histology to aid learning.

In Japan, a systematic screening protocol for the upper GI tract has been developed, although this is considered too complex for routine clinical practice. ${ }^{133}$ This was revised by the Japanese Society of Gastroenterological Cancer Screening to a simplified, but still elaborate protocol. ${ }^{134}$ Yao has more recently simplified this further to propose as a minimum required quality standard a 'systematic screening protocol for the stomach'. ${ }^{135}$ This is a station-based approach whereby each area of the stomach is viewed and photographed in either a clockwise or counterclockwise manner. The 22 pictures are arranged according to the order of the procedure. Additional pictures are taken of lesions (Figure 2). A recent study from China found that training including a systematic inspection protocol with 20 photos increased the detection of early gastric cancer from $0.2 \%$ to $2.3 \% .^{136}$

An e-learning module has been developed to teach endoscopists how to diagnose early gastric cancer based on the characterisation of gastritis-like lesions, ulcerative lesions and polypoid lesions, the so-called GUP system. ${ }^{137138}$ The GUP system has been evaluated in an RCT involving 332 endoscopists in 27 countries, with a higher mean improvement rate in the e-learning group than that of the non-e-learning group. ${ }^{138}$ A further study clearly demonstrated the efficacy of an e-learning system in improving endoscopists' capabilities to diagnose early gastric cancer using magnification-narrow band imaging. ${ }^{139}$ Such validated training modules may be incorporated into any future quality improvement (QI) programmes aimed at improving diagnosis of gastric cancer.

We recommend that when either GA or GIM is recognised on WLE, a full systematic endoscopic examination of the whole stomach is performed, taking no less than $7 \mathrm{~min}$, with full photographic documentation of antrum, pylorus, incisura, lesser curve, greater curve, fundus and cardia. For patients without known risk factors for gastric cancer, we recommend a standardised high-quality endoscopy as defined in the UK quality in upper gastrointestinal endoscopy position statement.

Optical endoscopic diagnosis of the premalignant or early malignant stomach

How does one identify premalignant or early malignant lesions and ensure accurate documentation when reporting? Are there mucosal features that identify these lesions (including recognising the atrophic border)?

What histopathogical and imaging modalities are suggested for the staging of glandular premalignant and early gastric malignant lesions of the stomach?

GA and GIM may be detectable by WLE; however, the accuracy is poor. Therefore, we do not recommend establishing a diagnosis or risk stratification using WLE alone (evidence level: moderate quality; grade of recommendation: strong; level of agreement: 93\%).

We recommend IEE as the best imaging modality to accurately detect and risk-stratify GA and GIM (evidence level: moderate quality; grade of recommendation: strong; level of agreement: 100\%).

We recommend that endoscopic appearances on WLE suggestive of GA or GIM require escalation to high-resolution IEE and, where available, magnification endoscopy (Evidence level: low quality; grade of recommendation: strong; level of agreement: 100\%).

We recommend that the location and extent of GA and GIM should be clearly documented with photographic evidence. Endoscopic grading should be documented as distal gastric (affecting antrum or incisura-low risk) or proximal gastric (affecting the corpus with or without the antrum and incisura-high risk) (evidence level: low quality; grade of recommendation: strong; level of agreement: 93\%).

We recommend that endoscopic appearances on WLE of gastric dysplasia and early gastric cancer (differences in colour, loss of vascularity, slight elevation or depression, nodularity, thickening, and abnormal convergence or flattening of folds) require escalation to IEE and, where available, magnification endoscopy (evidence level: low quality; grade of recommendation: strong; level of agreement: 100\%).

We recommend IEE as the best imaging modality to accurately diagnose and stage gastric dysplasia and early gastric cancer (evidence level: moderate quality; grade of recommendation: strong; level of agreement: 100\%).

Endoscopic detection and staging of GA, GIM and dysplasia is achievable with high-resolution WLE but improved with image enhancement and magnification endoscopy. The white light, image-enhanced and magnification appearances of the different mucosal patterns within the stomach are described below, covering the normal antrum and corpus, as well as GA and GIM.

\section{Normal gastric appearances}

White light endoscopy

Typically, the surface of the normal corpus, when the stomach is empty and not distended, is almost invariably in folds, also called rugae, which vary in size depending on the degree of insufflation during the endoscopic assessment. In contrast, the surface of the normal fundus and antrum is smooth. The colour of the normal gastric mucosa, as indeed of the whole GI tract, is velvety, glossy dark rose or red with a regular arrangement of the collecting venules, usually visible as red spidery vessels 


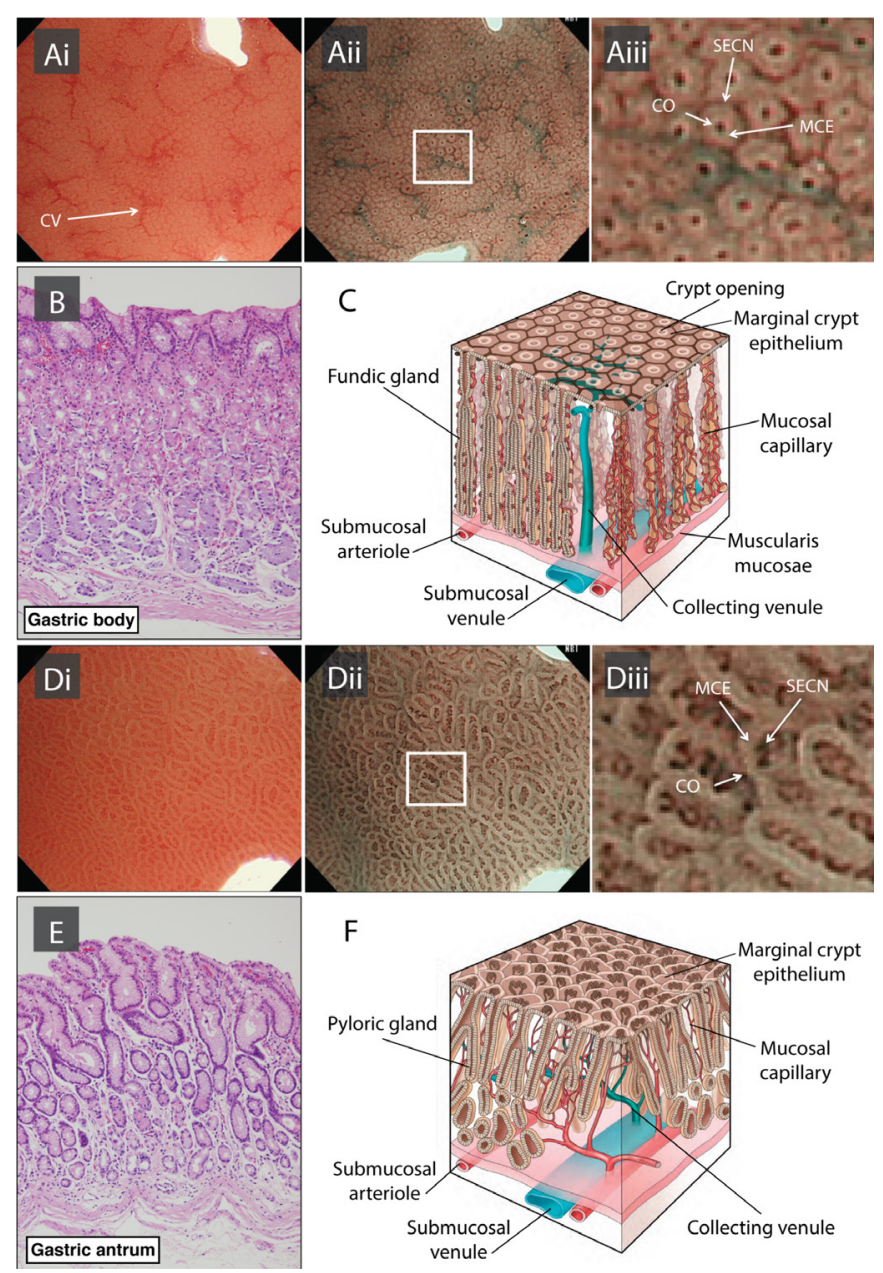

Figure 3 Normal gastric corpus and antrum mucosal surface pattern with white light, enhanced and magnification endoscopy. The round 'pit patterns' of the corpus ( $\mathrm{Ai}$ ) and elongated 'pit patterns' of the antrum (Di) can be seen without magnification or enhancement. In the corpus (Ai), the red collecting venules (CV) are evident as well as the round dark red crypt openings (CO). The vascular anatomy becomes more pronounced with NBI (Aii \& Dii). The visible anatomical components seen on magnification NBI include the dark brown 'crypt openings' (CO), the dark brown sub-epithelial capillary network (SECN), and the light brown marginal crypt epithelia (MCE). The corpus mucosa has dark round 'crypt openings', surrounded by the lighter MCE, followed by the darker circular SECN (Aii \& Aiii). In contrast, the dark, oblique 'crypt openings' in the antrum are grooved so the light coloured ridged or villiform epithelium (MCE) surrounds the dark SECN, termed the 'groove type pattern' (Dii \& Diii). The corresponding histopathological architecture can be seen in the corpus ( $B$ and $C$ ) and in the antrum ( $E$ and F).

in the normal corpus. ${ }^{140-143}$ The presence of these collecting venules is characteristic of a normal stomach without $H$. pylori (sensitivity 93\%, specificity 48\%). ${ }^{140} 144145$ With current white light, high-resolution endoscopes, the round 'pit patterns' of the corpus and elongated 'pit patterns' of the antrum can be seen without magnification or enhancement (Figure 3Di).

\section{Magnification and enhanced endoscopy}

The two key features that characterise the gastric mucosa on magnifying endoscopy include the surface structure and vascular architecture.
Essentially, the corpus mucosa has straight or tubular glands with round 'crypt openings'. ${ }^{146}$ These dark round pits are surrounded by the lighter coloured marginal crypt epithelia (MCE), then the darker circular subepithelial capillary network. This structure forms the typical foveolar type pattern, where dark areas (capillaries) surround light areas (glands). ${ }^{147-149}$ These appearances correspond with the tubular gland structure of the corpus mucosa histologically (Figure 3B \& C). In narrow band imaging (NBI), the pattern 'regular vessels with circular mucosa' is associated with normal histology (accuracy 83\%; 95\% CI 75\% to $90 \%){ }^{150}$

In contrast to the corpus, the glands in the antrum are oblique and branching and the dark 'pit openings' are grooved. The light-coloured ridged or villiform epithelium (MCE) surrounds the dark subepithelial capillary network, which can be seen as coiled vessels (Figure 3Aii Aiii). This appearance is termed the 'groove-type pattern'. ${ }^{147} 148$ These appearances correspond with the papillary surface structure of the antral mucosa histologically (Figure 3E \& f).

\section{Chronic $\boldsymbol{H}$. pylori gastritis}

A number of endoscopic features are suggestive of chronic $H$. pylori gastritis, including the absence of collecting venules, antral nodularity, enlarged gastric folds, enlargement and destruction of the gastric glands, sticky tenacious adherent mucous, turbid gastric juice, and xanthomas. ${ }^{140-142}$ Loss of collecting venules and capillary vascular structure was correlated with chronic inflammation and activity. With progression of mucosal atrophy, irregular collecting venules become visible. ${ }^{151}$

\section{Gastric atrophy}

Four principal endoscopic features of GA are described by Nakayama et al, Uedo et al and Yao et al ${ }^{152-154}$ : pallor, loss of gastric folds, prominence of the vessels and the atrophic border (Figure 4). Increased visibility of the vascular network showed a sensitivity of $48 \%$ and a specificity of $87 \%$, while the loss of gastric folds has a sensitivity of $67 \%$ and specificity of $85 \% .{ }^{155}$

Long-term cohort studies suggest that the Kimura-Takemoto classification is a useful risk stratification assessment tool to predict gastric adenocarcinoma development. ${ }^{26} 31156$ Essentially this tool uses the extent of the atrophic border (the border between the pale atrophy and normal red-coloured stomach) to stage the extent of GA (Figure 4). In a cross-sectional cluster sampling historical study between the UK and Japan, endoscopic grading was shown to be comparable to histopathology and correctly predict histopathological atrophy with few false-negative results. ${ }^{157158}$ This work needs further confirmation in a larger setting outside of Japan. The Kimura-Takemoto classification has been simplified to a modified staging system involving the antrum only (antral), antrum to incisura (antral dominant), antrum to lesser curve (corpus dominant), and antrum, lesser curve and greater curve (pan-atrophy). This staging system integrates the Sydney biopsy system discussed later (Figure 5). The GDG agreed that the extent of GA and GIM should be stratified as low risk (involving the antrum and incisura) or high risk (involving the corpus and the antrum/incisura or the corpus alone).

\section{Gastric intestinal metaplasia} White light endoscopy

GIM typically appears as small grey-white slightly elevated plaques surrounded by mixed patchy pink and pale areas of mucosa causing an irregular uneven surface (figure 6A). 


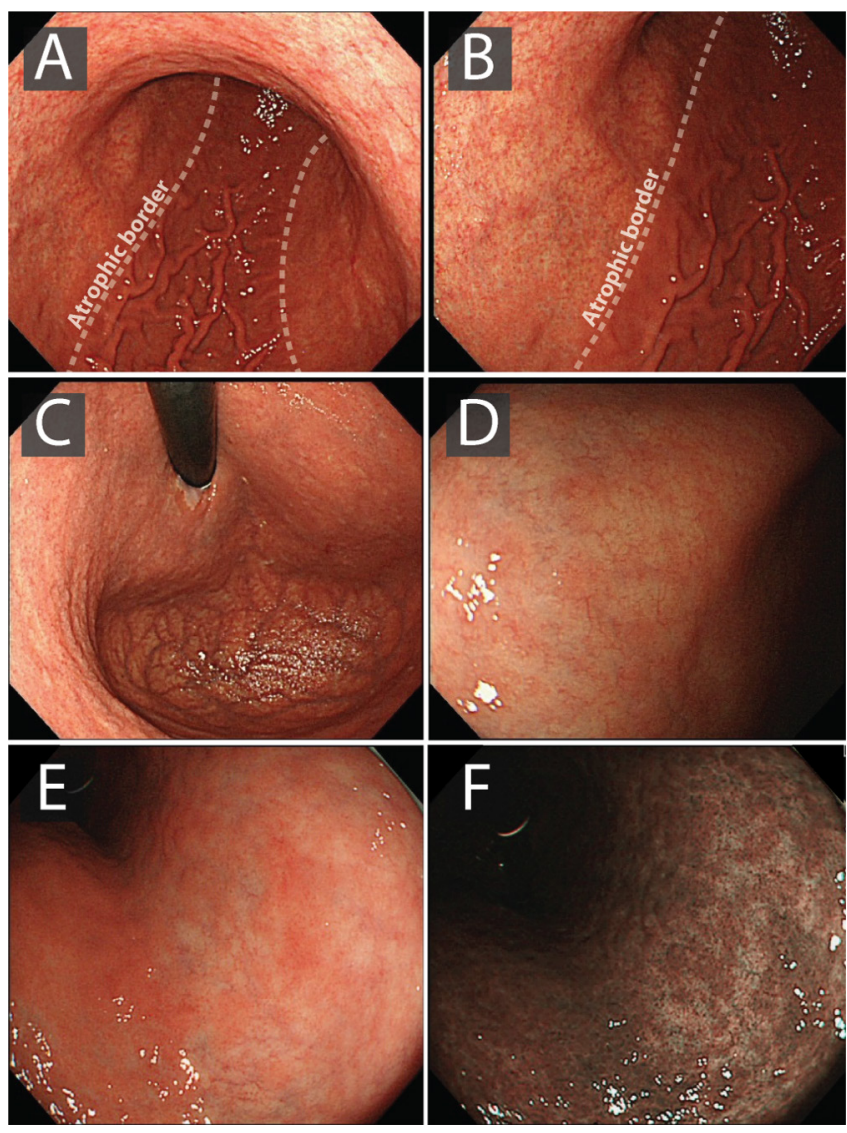

Figure 4 Chronic atrophic gastritis (CAG) and the atrophic border on white light and image enhanced endoscopy. There are four principle endoscopic features of CAG: palor ( $A, B, C, D$ and $E)$, loss of gastric folds $(A, B, C, D$ and $E)$, prominence of the vessels $(A, B, C$ and $D)$ and the atrophic border ( $A$ and $B$ ). The paler areas of atrophy are also clear on image enhancement $(F)$.

Mottled patchy erythema has also been positively associated with GIM. ${ }^{159}$ However, diagnosis using standard endoscopy alone (without high-resolution or enhanced imaging) is unreliable. ${ }^{160-165}$

\section{Magnification and IEE}

As patches of GIM expand, the straight/tubular glands of the corpus elongate to a 'groove-type pattern' similar to that of the antrum or villiform pattern of the intestine (figure $6 \mathrm{C}$ D). Although these changes can easily be distinguished from the normal mucosal background in the corpus both with high-resolution white light and enhanced endoscopy, GIM in the antrum is more difficult to characterise as the pre-existent mucosal architecture is quite similar and also appears oblique and grooved. ${ }^{148} 166$ Additional features of GIM include the light blue crest (LBC) and the marginal turbid band (MTB) ${ }^{167}$ and the white opaque substance (lipid droplets) obscuring the subepithelial capillaries. ${ }^{168}$ The LBC is a fine, blue-white line on the crest of the mucosal surface seen with NBI enhancement and is a highly specific sign of the presence of GIM ${ }^{166169}$ (figure 6E F). The MTB is a white turbid band on the mucosal surface. It is suggested that the MTB may represent a sign of early GIM, whereas the LBC appears with progression to severe GIM, although whether this is reproducible remains uncertain.
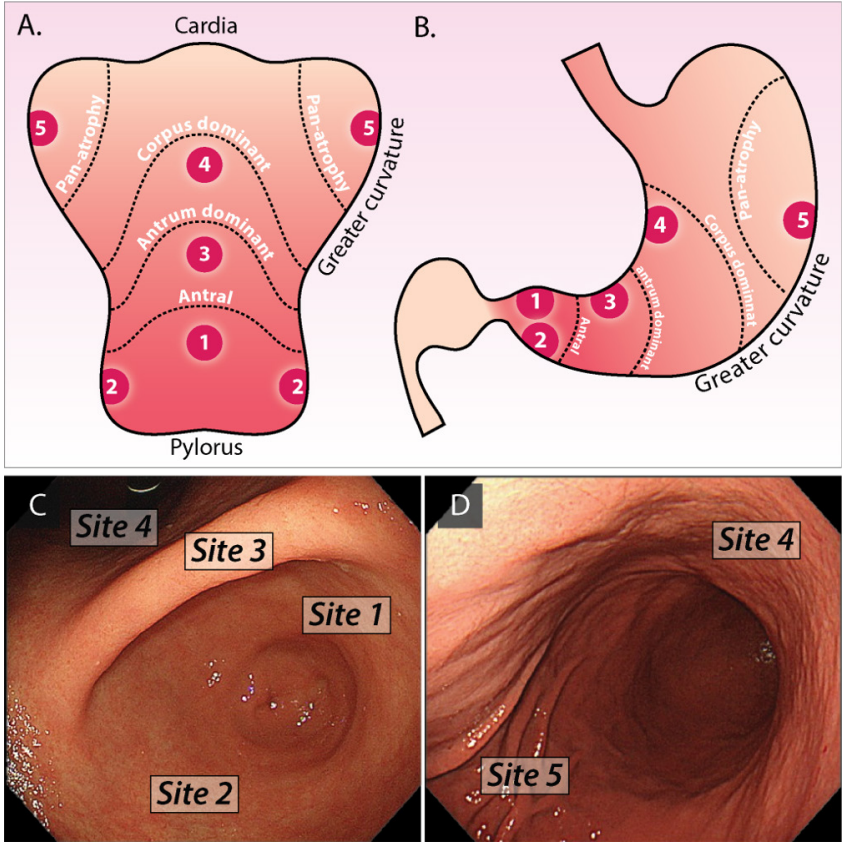

Figure 5 The Integrated and Modified Kimura \& Sydney Biopsy System. The modified Kimura staging system divides the extent of atrophy into antrum only (antral), antrum to incisura (antral dominant), antrum to lesser curve (corpus dominant), and antrum, lesser curve and greater curve (pan-atrophy). This system integrates Sydney protocol biopsies which should be taken from the antrum (site 1 and 2), incisura (site 3), lesser curve (site 4) and greater curve (site 5). The anatomical CAG boundaries and biopsy sites can be seen in the splayed (A) and cross-sectional cartoon (B) of the stomach. The biopsy sites defined in the endoscopic retroflexed (C) and forward view (D).

\section{Optimal techniques for the detection and classification of GA and GIM}

Most studies have demonstrated improved accuracy of enhanced and magnification imaging in the classification and detection of GA, GIM, dysplasia and cancer, in comparison with WLE. ${ }^{150} 162163165170171$ An overview of enhanced imaging studies with various modalities and their performance characteristics is provided in table 4.

A simplified classification system using NBI without magnification by Pimentel-Nunes et al has been shown to be accurate and reliable for the diagnosis of GIM and dysplasia. ${ }^{150}$ In the validation study a tubulovillous mucosal pattern was associated with GIM (accuracy 84\%). Irregular vessels and mucosal pattern were associated with dysplasia (accuracy 95\%). The LBC finding was moderately reliable $(k=0.49)$, but very specific $(96 \%)$ for GIM. In a recent study by Kanemitsu et al, ${ }^{168}$ the sensitivity and specificity of LBC for histologically diagnosed intestinal metaplasia were $62.5 \%$ and $93.8 \%$, respectively. The sensitivity and specificity of white opaque substance were $50.0 \%$ and $100.0 \%$ (95\% CI $85.0 \%$ to $100.0 \%$ ), respectively. The combination of LBC and white opaque substance improved the overall sensitivity up to $87 \%$ and $93.8 \%$.

A second prospective multicentre study compared WLE with WLE plus NBI. ${ }^{162}$ NBI demonstrated a high concordance with histopathological diagnosis, superior to standard WLE. However, it is important to note that this study assessed WLE plus NBI, rather than NBI alone, and although high-resolution WLE had a good overall sensitivity of $85 \%$ for all pathology, this decreased to $53 \%$ for the detection of GIM. NBI versus WLE 
increased the sensitivity for the diagnosis of intestinal metaplasia significantly $(87 \%$ vs $53 \% ; \mathrm{p}<0.001)$ and for the diagnosis of dysplasia (92\% vs $74 \%)$. This study suggests that WLE alone is therefore not sufficiently accurate for GIM detection. Similar comparative results have been demonstrated in a recent prospective blinded trial. ${ }^{165}$

The sensitivity and specificity of WLE for the histological diagnosis of GA were reported to be $61.5 \%$ and $57.7 \%$, respectively, in the antrum, and $46.8 \%$ and $76.4 \%$, respectively, in the body of the stomach. ${ }^{170}$

Compared with WLE, NBI combined with magnifying endoscopy can also effectively diagnose early gastric adenocarcinoma. ${ }^{171}$

Thus, in summary, the GDG agreed that WLE alone was not sufficiently accurate to reliably diagnose GA or GIM, and enhanced optical techniques should be used for diagnosis and staging.

A scale for endoscopic staging of GIM using NBI was created and returned an area under the curve (AUC) of 0.98 for WLE followed by NBI for diffuse GIM. ${ }^{162}$ This was externally validated by the same group, and for a diagnosis of OLGIM III/ VI the AUC was 0.96 (95\% CI 0.93 to 0.98). This endoscopic grading of GIM was $89 \%$ sensitive and $95 \%$ specific for a risk stratification of moderate to severe GIM if a cut-off score of $>4$ was used. ${ }^{172}$ On this basis, it could be argued that endoscopic staging with high-resolution WLE plus NBI is sufficiently accurate for diagnosis and staging. This is an area of active future research.

\section{Gastric dysplasia and early gastric cancer}

Detection of gastric dysplasia and early gastric cancer is notoriously difficult due to the often only subtle findings and the lack of well-defined endoscopic appearances under white light inspection. Features commonly described, but not exhaustive, include differences in colour (ie, more red or pale), loss of vascularity, slight elevation or depression, nodularity, thickening, and abnormal convergence or flattening of folds. ${ }^{173} 174$

Therefore, optimal clearing of mucous and secretions is essential to allow for continuous and meticulous search of areas with features different from the surrounding mucosa. ${ }^{175} 176$

Recourse to chromoendoscopy with indigo carmine solution $(0.2 \%)$ or virtual chromoendoscopy (NBI, flexible spectral imaging colour enhancement (FICE), i-Scan, blue laser imaging) is commonly advocated to enhance contrast and visualisation of areas of concern or mucosal abnormalities. Areas of dysplasia may present throughout the stomach, with a slight predominance in the antrum and along the lesser curvature, and can vary in size from a few millimetres to several centimetres.

Gastric dysplasia can be morphologically classified into adenomatous (intestinal), which includes adenomatous polyps; foveolar (gastric); and hybrid type. ${ }^{47} 177$ Compared with the adenomatous type, the foveolar type appears to be more commonly associated with HGD. ${ }^{47} 178$ Endoscopically, these lesions are usually detected as 0-Is, 0-IIa or 0-IIc types according to the Paris classification of superficial neoplastic lesions. ${ }^{175}$

Adenomatous dysplasia is more likely to occur in the gastric body and lesser curvature of the stomach, whereas foveolar dysplasia is more typically located in the gastric antrum and incisura angularis. In addition, foveolar-type lesions are smaller, often reddish in colour and present as flat or depressed areas more frequently than the adenomatous type. ${ }^{47}$
Biopsy strategy for diagnosis and risk stratification of the premalignant stomach

What biopsy strategy should be adopted for the sampling and reporting of the premalignant and early malignant stomach?

Should all those undergoing routine endoscopy be biopsied or are there groups to target?

We recommend that patients with image-enhanced features of CAG should undergo biopsies for confirmation of endoscopic diagnosis; biopsies are directed at mucosal sites within Sydney protocol areas where enhanced imaging discloses GIM. Biopsy samples should be collected in separate containers and labelled as either 'directed' or 'random' to corroborate endoscopic staging assessment (evidence level: low quality; grade of recommendation: strong; level of agreement: 93\%).

We suggest that a baseline endoscopy with biopsies should be considered in individuals aged $\geq 50$ years, with laboratory evidence of pernicious anaemia, defined by vitamin $B_{12}$ deficiency

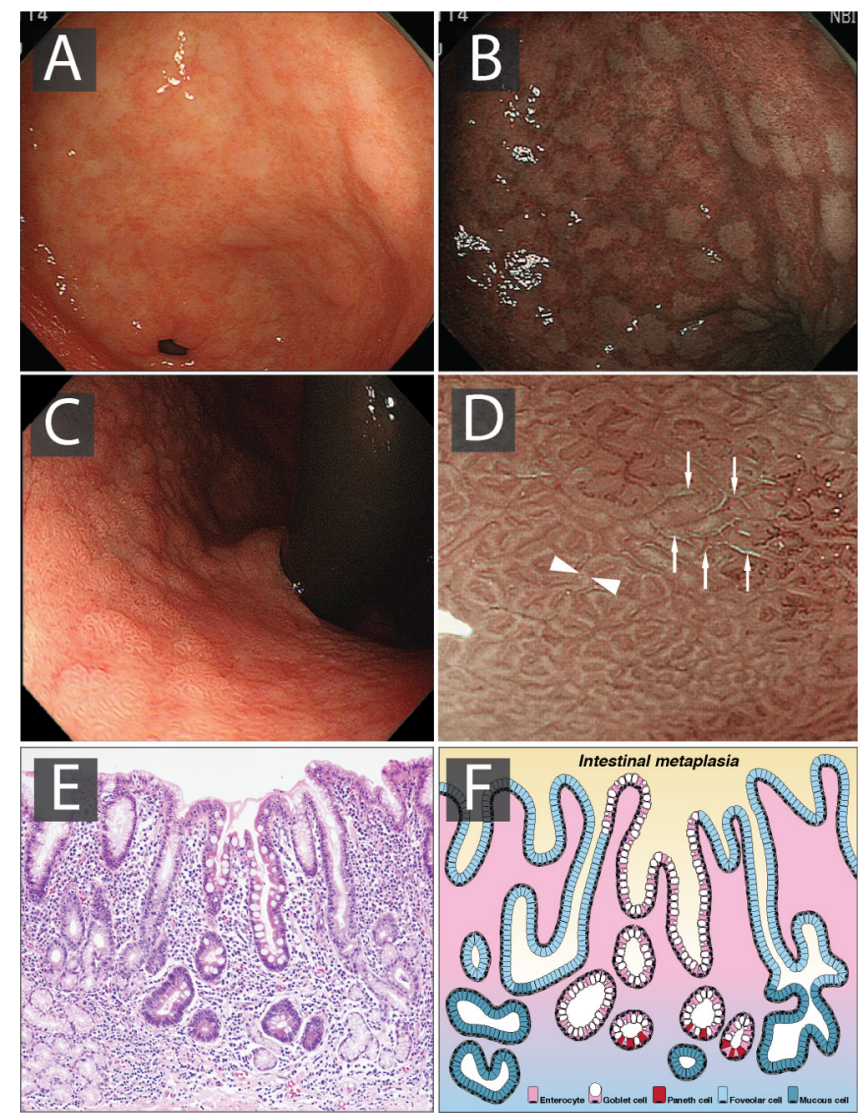

Figure 6 Gastric intestinal metaplasia under white light, image enhanced and magnification endoscopy. Intestinal metaplasia typically appears as small grey-white slightly elevated plaques surrounded by mixed patchy pink and pale areas of mucosa causing an irregular uneven surface (A). These appearances are more evident with image enhancement (B). Corpus GIM can be distinguished from the normal straight/tubular glands of the corpus by a 'groove type pattern' similar to that of the antrum or villiform pattern of the intestine and may be appreciated with higher resolution technology on white light endoscopy (C). GIM in the antrum is more difficult to characterize as the normal glands are oblique. Additional features of GIM to aid diagnosis in the antrum include the light blue crest (LBC) and the marginal turbid band (MTB) (D). The LBC is a fine, blue-white line on the crest of the epithelial surface seen with NBI enhancement (Fine arrows in D). The MTD can be seen between the broad arrows. The numerous goblet cells characterise GIM (E \& F). 


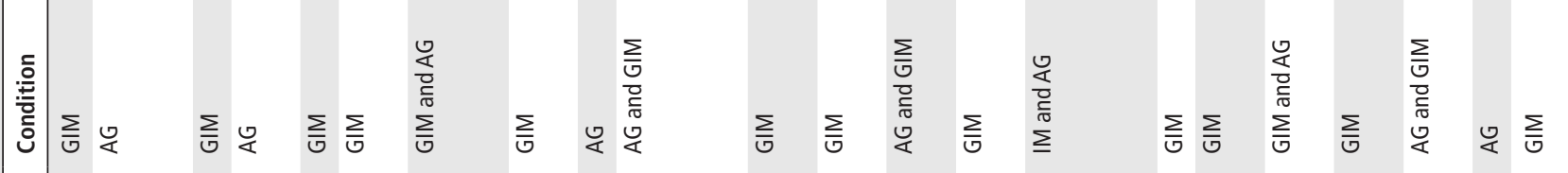

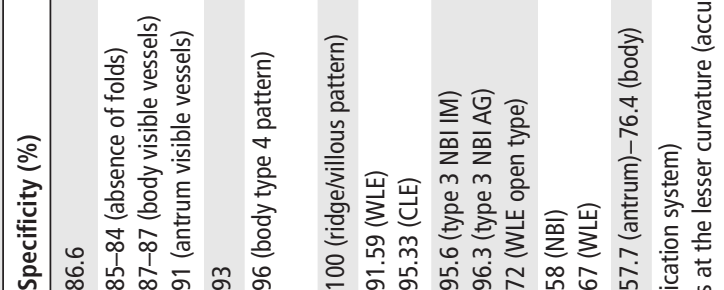

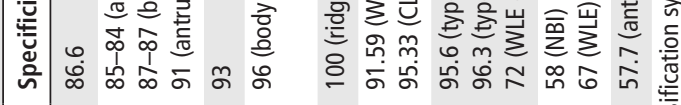

莺 辛

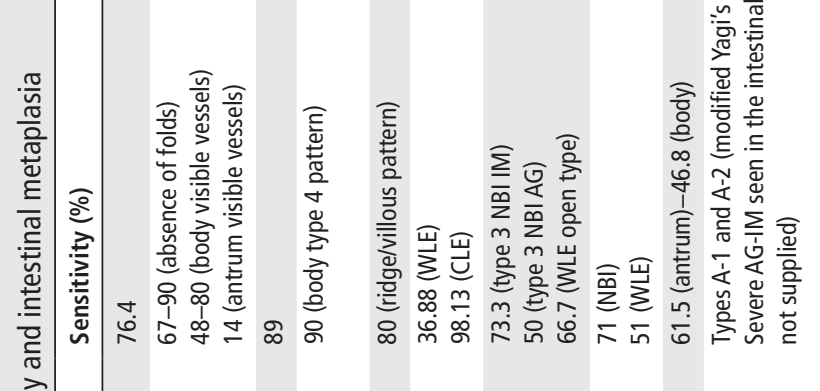

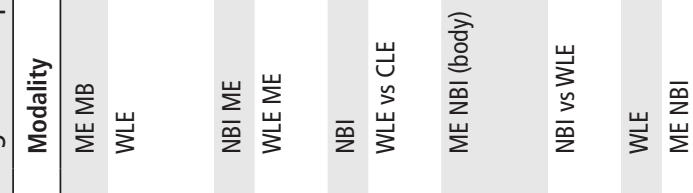

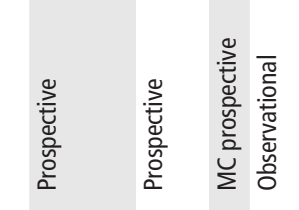

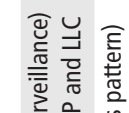

恙畓言

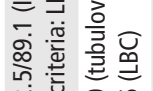

我

产品

$8 \%$

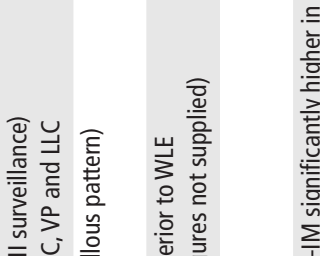

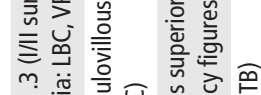

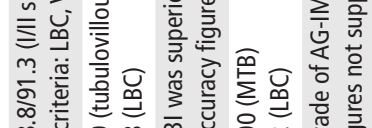

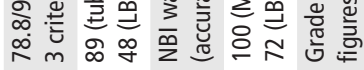

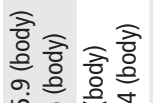

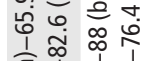

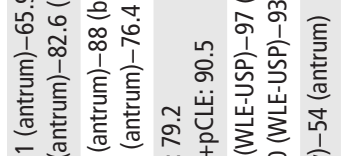

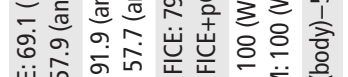

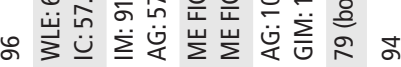

$$
\text { ه }
$$

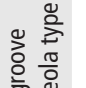

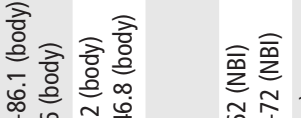

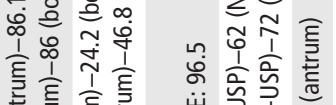

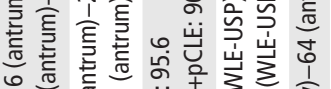

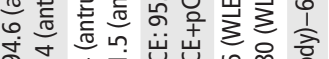

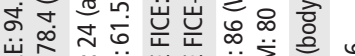

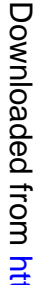

$\stackrel{\widehat{Q}}{\leftrightarrows}$

의

응 $\stackrel{m}{=}$ \& $\stackrel{\sim}{\sim} \underset{\sim}{\sim}$ 


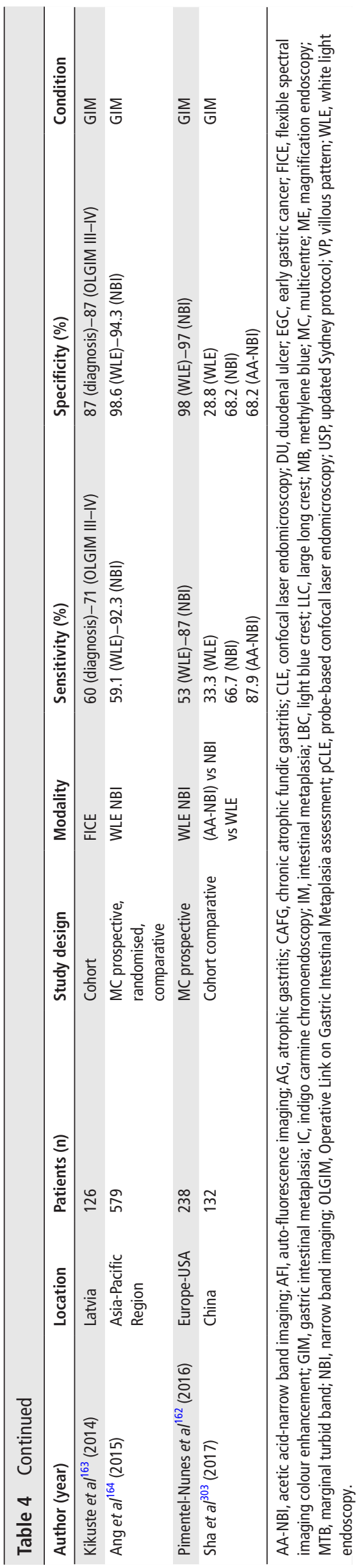

and either positive gastric parietal cell or intrinsic factor antibodies. As GA affects the corpus in pernicious anaemia, biopsies should be taken from the greater and lesser curves (evidence level: low quality; grade of recommendation: weak; level of agreement: 93\%).

Biopsies should be undertaken in patients with endoscopic suspicion of GA, GIM or early neoplasia.

Current surveillance protocols stipulate random biopsies in specified locations according to the updated Sydney protocol (Figure 5). However, random sampling does not reliably foster correlation of endoscopic and histopathological findings and carries a risk of sampling error. By contrast, 'directed' biopsies of mucosal foci suspicious for GIM in areas of the updated Sydney protocol will drive quality control and ultimately propel endoscopy-led staging and risk stratification. This targeted biopsy strategy links endoscopic GIM detection with the well-established OLGIM histopathological classification scheme. The aforementioned numerical endoscopic classification system for staging of GIM has been shown to correlate strongly with OLGIM and with the extent of GIM ${ }^{150}$ further supporting this change in practice. The GDG did not agree on whether OLGA or OLGIM should be routinely used, principally because there is insufficient expertise or capacity for this to be routinely undertaken. Individual units, however, may choose to adopt this risk stratification system.

\section{SURVEILLANCE}

Should individuals with gastric premalignant lesions undergo surveillance?

We recommend endoscopic surveillance every 3 years should be offered to patients diagnosed with extensive GA or GIM, defined as that affecting the antrum and body (evidence level: low quality; grade of recommendation: strong; level of agreement: 100\%).

We do not recommend surveillance in patients with GA or GIM limited just to the gastric antrum; unless there are additional risk factors such as a strong family history of gastric cancer or persistent $H$. pylori infection, then we suggest 3-yearly surveillance (evidence level: low quality; grade of recommendation: strong; level of agreement: 93\%).

We recommend that patients with non-visible LGD should undergo an immediate second endoscopy with enhanced imaging and extensive biopsy sampling, followed by a repeat endoscopy within 1 year if no visible neoplasia is detected. If there is persistent, non-visible LGD, endoscopy should be repeated annually thereafter (evidence level: low quality; grade of recommendation: strong; level of agreement: 100\%).

We recommend that patients with non-visible HGD should undergo an immediate second endoscopy with enhanced imaging and extensive biopsy sampling. We recommend ongoing surveillance at 6-monthly intervals for persistent, non-visible HGD. HGD should be discussed at the regional upper GI cancer $M D T$ and referred to a clinician with the appropriate expertise (evidence level: low quality; grade of recommendation: strong; level of agreement: 100\%).

\section{Surveillance of CAG}

As the neoplastic cascade follows a multistep process from $H$. pylori-associated gastritis through GA and GIM to dysplasia, ${ }^{179}$ it follows that surveillance of a high-risk population may lead to the detection of early gastric cancer. Furthermore, the evolution of endoscopic techniques such as ESD, with 5-year disease-free 
survival rates of $99 \%,{ }^{180}$ further supports the detection of early gastric cancer through surveillance.

Several factors influence the risk of progression to cancer, including the extent of atrophy and GIM and a family history of gastric cancer. A strong family history is defined as those with a first-degree relative or two or more second-degree family members with histologically proven gastric adenocarcinoma. Those at the highest risk with GIM in the antrum and corpus have a $9.8 \%$ cumulative 5 -year incidence of cancer. ${ }^{26}$

\section{Outcomes of endoscopic surveillance of premalignant conditions}

No randomised controlled studies on the benefit of GIM surveillance have been carried out. There are, however, observational data. A small UK study assessed the effect of endoscopic surveillance of patients with GIM (specifically type III, incomplete GIM) every 6-12 months over a 12 -year period. Of the 26 patients surveyed, 11 developed gastric adenocarcinoma, which was limited to the mucosa or submucosa at the time of detection. Although four patients were lost to follow-up, in comparison with the unit as a whole, the proportion of early cancers was much greater in those patients surveilled. ${ }^{181}$

In a second study 166 patients with GIM, dysplasia, GA, ulcers or polyps were surveyed over 10 years and compared with 1753 open-access endoscopy patients. Compared with the open-access group, there were more stage I/II cancers (76\% vs 23\%) and an improved 5 -year survival (50\% vs $10 \%)$ in the surveillance group. Of the patients surveilled, 93 had GIM, of whom 10 developed gastric adenocarcinoma. In patients with GA and GIM, the risk of malignancy was $11 \% .{ }^{182}$ Both the aforementioned studies, however, were conducted before the advent of high-resolution endoscopy and IEE, which makes it difficult to extrapolate to current practice.

A northern European study in low-incidence gastric cancer areas surveyed 279 patients with GA, GIM or dysplasia over a mean period of 57 months. Of these, $1.4 \%$ (four patients) were diagnosed with HGD or gastric cancer, of whom two were successfully treated by ESD and two by gastrectomy. All four patients whose disease progressed had extensive GIM as defined by the OLGIM stage. None of those with limited GA or GIM progressed. The overall risk of neoplastic progression was $0.3 \%$ a year. ${ }^{183}$

A number of studies have evaluated surveillance in pernicious anaemia and body-predominant atrophic gastritis; however, only one study has demonstrated the development of gastric cancer in 2 of 56 patients at the time of a surveillance endoscopy, 3 years after the initial endoscopy.

The MAPS guidelines published in 2012 recommend that patients with extensive GA and/or extensive GIM should be offered endoscopic surveillance every 3 years. ${ }^{10}$ MAPS also recommends that patients with mild to moderate atrophy or GIM limited to the antrum do not need follow-up.

\section{Cost-effectiveness of endoscopic surveillance of premalignant conditions}

Early studies examining the cost-effectiveness of CAG surveillance have obtained conflicting results, which is partly due to the widely ranging variation in prevalence and progression to cancer. Modelling undertaken by Yeh et al in 2010 in a US population found surveillance to be cost-effective for men over 50 years with dysplasia treated by EMR with annual surveillance (cost per quality-adjusted life-year (QALY) was US\$39 800), although for 10-yearly surveillance in those with any GIM (US\$544 500). ${ }^{184} \mathrm{~A}$ more recent cost-utility economic analysis in a European population aged between 50 and 75 years aimed to assess the cost-effectiveness of endoscopic screening of the premalignant stomach using Markov modelling. ${ }^{185}$ The primary outcome was the incremental cost-effectiveness ratio (ICER) of a 3-yearly endoscopic surveillance versus no surveillance. Endoscopic surveillance every 3 years provided an ICER of $€ 18336$, well below the adopted threshold of $€ 36575$ corresponding to the proposed guideline limit of US\$50000. Moreover, robustness analysis showed that in $78 \%$ of examined scenarios the model was cost-effective. Three-yearly surveillance was found to be more cost-effective than 5-10 yearly strategies. Two further studies from low to intermediate risk populations in Singapore using Markov modelling and cost-utility analysis found 1-yearly and 2-yearly surveillance were cost-effective, although 3 -yearly intervals were not analysed. ${ }^{186} 187$ In conclusion, recent cost-effectiveness models support surveillance in CAG in populations at low to intermediate risk between 1-yearly and 3-yearly. These models did not investigate those with extensive CAG, where the risk of progression is greater and thus the cost-effectiveness is likely to be superior.

As the cancer risk of focal intestinal metaplasia of the cardia is poorly defined and appears much lower than short-segment Barrett's oesophagus we do not recommend surveillance in this group. ${ }^{43}$

\section{Summary}

Although there is a lack of randomised data on the survival benefits of surveillance for GIM, there is moderate evidence demonstrating progression of premalignant conditions, particularly extensive GIM, to gastric adenocarcinoma, and evolving data from surveillance studies. The cost-effectiveness data are also compelling. We do accept, however, as with surveillance of Barrett's oesophagus, that surveillance of GA and GIM will remain controversial. We recommend a surveillance interval of 3 years for those patients with extensive GA or GIM defined as that affecting the antrum and body. However, surveillance may not be appropriate for all patients with extensive atrophy and GIM, particularly the very elderly and those with multiple comorbidities where the benefit of surveillance may be offset by the risks of diagnostic endoscopy. For those with GA or GIM limited to the antrum, but with additional risk factors such as a family history of gastric adenocarcinoma and persistent $H$. pylori infection, we also recommend a surveillance interval of 3 years. Persistent $H$. pylori infection is defined as that refractory to treatment. We also suggest, where possible, as with Barrett's oesophagus surveillance, that endoscopy is undertaken on a dedicated screening list. The remit of these guidelines do not cover the management of HDGC. ${ }^{63}$

\section{Surveillance of dysplasia}

Although the risk of progression of dysplasia to gastric adenocarcinoma varies between studies, it is clear that HGD confers a greater risk of progression than LGD. Of patients with HGD, $30 \%$ to $85 \%$ progress to cancer with follow-up periods of up to 5 years with an estimated annual incidence of $6 \% .^{25} 44$ The overall risk of malignancy for LGD (combining mild and moderate dysplasia, as described in earlier classification systems) varies from $0 \%$ to $33 \%$ over follow-up periods of up to 66 months. ${ }^{188-194}$ Two observational studies have shown progression rates of between 3\% and 9\%. ${ }^{190} 191$

Dysplasia in random biopsies also appears to increase the risk of cancer, possibly by as much as $6 \%$ a year. ${ }^{15} 25$ It is important to note that in those patients found to have incidental HGD 
or cancer by random biopsies on the index endoscopy, $80 \%$ will have a detectable lesion by virtual chromoendoscopy. ${ }^{195} \mathrm{~A}$ finding of gastric dysplasia also carries a high risk of synchronous gastric adenocarcinoma, with rates as high as 30\% in some studies. $^{196}$

The histopathological biopsy diagnosis may not be representative of the final histopathological grade on excision. This has been described in a meta-analysis of 16 studies including over 3000 patients. Of patients initially diagnosed with LGD on biopsy, 25\% were subsequently diagnosed with more advanced lesions after endoscopic resection. ${ }^{197}$ Of these, $16.7 \%$ were HGD and $6.9 \%$ were invasive lesions. A Korean series demonstrated a similar upgrade of diagnosis from LGD in $18.7 \%$ of cases. ${ }^{198}$ Thus, the rate of underdiagnosis is not insignificant. These data, however, are discordant with the lower documented progression rates in larger series of up to $9 \%$. The authors found that lesion size $>2 \mathrm{~cm}$, surface depression, absence of whitish discolouration, the presence of spontaneous bleeding and nodularity on endoscopic examination were the major risk factors associated with a higher histopathological grade on excision. ${ }^{197} 198$ In those patients who undergo surveillance, repeat or multiple biopsies appear to not substantially improve the accuracy of the initial diagnosis. $^{199} 200$

Cases that are indefinite for dysplasia may also be understaged or overstaged, with up to $25 \%$ upstaged to dysplasia or cancer. $^{201} 202$ We therefore recommend that patients who are indefinite for dysplasia are reviewed by an expert pathologist and undergo a repeat procedure with image enhancement.

Based on the current evidence, there are clear grounds to support the endoscopic resection of visible LGD and HGD, providing that the risk of endoscopic resection is justifiable for health reasons and is the preference of the patient. We suggest surveillance for HGD and LGD only if resection is not possible or appropriate, or if the dysplasia is not visible, or is the preference of the patient. For patients with LGD undergoing surveillance, we recommend surveillance intervals of 1 year with high-quality systematic endoscopy. If biopsies are negative for dysplasia after three consecutive endoscopies, then consider 3-yearly surveillance. For those with HGD undergoing surveillance, we recommend a repeat high-quality endoscopy, repeated 6-monthly thereafter. All visible dysplasia should be resected where appropriate. There are no cost-effectiveness studies on surveillance of either HGD or LGD, and it is unclear for how long surveillance should be continued. For those patients found to have non-visible dysplasia on random biopsies, we suggest an immediate systematic endoscopy with enhanced imaging, if necessary in an expert centre.

\section{Surveillance after EMR and ESD for dysplasia and early gastric cancer}

The risk of neoplastic synchronous or metachronous lesions as well as recurrence after gastric ESD or EMR is high and varies between $10 \%$ and 20\%. ${ }^{203-206}$ Therefore endoscopic surveillance is necessary. This will allow further diagnosis of early neoplastic lesions, which can then be removed endoscopically. Few data on the follow-up interval are available; however, most recurrences occur within the first year after resection. ${ }^{203} 207-210$ We suggest therefore that a follow-up endoscopy is undertaken 6 months after ESD or EMR of neoplasia, and if no lesions are identified, annually thereafter. This is in line with the Japanese and ESGE guidelines. $^{211-213}$

\section{Treatment: endoscopic therapy}

What lesions are amenable to endoscopic removal?

How should these lesions be removed?
Are there criteria on histopathological assessment that determine prognosis and follow-up?

We recommend that all gastric dysplasia and early gastric adenocarcinoma should be resected en bloc (an EMR technique can achieve en bloc excision for lesions $\leq 10 \mathrm{~mm}$ in size, but only an ESD technique can ensure en bloc excision for lesions $>10 \mathrm{~mm}$ in size) (evidence level: high quality; grade of recommendation: strong; level of agreement: 100\%).

We recommend that complete $(R 0)$ endoscopic resection of gastric dysplasia and early gastric adenocarcinoma with the following features should be considered as curative:

1. LGD.

2. HGD.

3. Well or moderately differentiated intramucosal adenocarcinoma, irrespective of size and without ulceration.

4. Well or moderately differentiated intramucosal adenocarcino$m a,<3.0 \mathrm{~cm}$ in size if ulcerated.

5. Well or moderately differentiated submucosal adenocarcinoma, $<3.0 \mathrm{~cm}$ in size, with superficial submucosal invasion (Sm1; <500 $\mu m$ submucosal invasion as measured in a straight line from the deepest fibre of the muscularis mucosae).

6. Poorly differentiated intramucosal adenocarcinoma, $\leq 2.0 \mathrm{~cm}$ in size (evidence level: moderate quality; grade of recommendation: strong; level of agreement: 93\%).

The histopathological features of early gastric adenocarcinoma associated with a higher risk of LNM after endoscopic resection include the following:

1. Poorly differentiated submucosal cancer, irrespective of invasion depth below muscularis mucosae.

2. Signet ring cancer.

3. Lymphovascular invasion.

4. Depth of submucosal invasion $\geq 500 \mu m$ as measured in a straight line from the deepest fibre of the muscularis mucosae (evidence level: moderate quality; grade of recommendation: strong; level of agreement: 93\%).

We suggest that, where possible, all cases considered for resection should be discussed in an MDT with the appropriate expertise, including pathologists and therapeutic endoscopists. When there is no local expertise, patients should be referred to an expert centre. Before any therapeutic procedure is undertaken, the risks and benefits of endoscopic resection and surgery should be discussed with the patient to aid their decision-making.

These recommendations apply to the intestinal type of gastric cancer as defined by the Lauren classification. ${ }^{214} 215$ Diffuse-type adenocarcinoma carries a worse prognosis than the intestinal type, which appears to be independent of the $\mathrm{T}$ and $\mathrm{N}$ stage. ${ }^{215}$

The risk of LNM underpins endoluminal therapy for early gastric adenocarcinoma. This risk has to be weighed against the significant risk of morbidity and mortality following surgical resection. Endoscopic resection has become the preferred organ-preserving treatment for superficial gastric neoplastic lesions because of the low risk of LNM that these lesions portend. In a large series from the Far East, Gotoda et al reviewed the prevalence of LNM in 5265 gastrectomy specimens. On multivariate analysis they found that none of the 979 non-ulcerated lesions had LNM. Additionally, they found that none of the 145 well or moderately differentiated adenocarcinomas measuring $<30 \mathrm{~mm}$, those with submucosal invasion of $<500 \mu \mathrm{m}(\mathrm{Sm} 1)$, and those without lymphovascular invasion showed LNM. ${ }^{216}$ This supported the initial Japanese guidelines on the indication criteria for endoscopic resection of early gastric adenocarcinoma, which included intestinal-type adenocarcinoma, endoscopically diagnosed intramucosal cancer, lesion 


\begin{tabular}{|c|c|c|c|}
\hline \multicolumn{4}{|c|}{ Weighted scores for risk factors } \\
\hline \multicolumn{2}{|l|}{ Risk factors } & \multicolumn{2}{|l|}{ Points } \\
\hline \multicolumn{2}{|l|}{ Lymphatic invasion } & \multicolumn{2}{|l|}{3} \\
\hline \multicolumn{2}{|l|}{ Lesion size >30 mm } & \multicolumn{2}{|l|}{1} \\
\hline \multicolumn{2}{|l|}{ Positive vertical margin } & \multicolumn{2}{|l|}{1} \\
\hline \multicolumn{2}{|l|}{ Venous invasion } & \multicolumn{2}{|l|}{1} \\
\hline \multicolumn{2}{|c|}{ Submucosal invasion $\geq 500 \mu \mathrm{m}$} & \multicolumn{2}{|l|}{1} \\
\hline $\begin{array}{l}\text { Lymph node metastasis } \\
\text { (LNM) risk groups }\end{array}$ & Points & Risk of LNM (\%) & $\begin{array}{l}\text { Cancer-specific } \\
5 \text {-year survival (\%) }\end{array}$ \\
\hline Low & $0-1$ & 2.5 & 99.6 \\
\hline Intermediate & $2-4$ & 6.7 & 96.0 \\
\hline High & $5-7$ & 22.7 & 90.1 \\
\hline
\end{tabular}

size of $\leq 20 \mathrm{~mm}$ and non-ulcerated lesions. ${ }^{212}$ In a second large series, Hirasawa et al reviewed 3843 patients who underwent gastrectomy with lymph node dissection for poorly differentiated adenocarcinoma. On multivariate analysis they found that lesion size of $>20 \mathrm{~mm}$, lymphovascular invasion and submucosal involvement were independent risk factors for LNM. ${ }^{217}$

Overall the two large series on surgically resected early gastric cancer demonstrate that the risk of LNM of superficial lesions is small $(<1 \%)$ if the following criteria are met:

1. Well or moderately differentiated intramucosal adenocarcinoma, irrespective of size and without ulceration.

2. Well or moderately differentiated intramucosal adenocarcinoma, $\leq 3.0 \mathrm{~cm}$ in size if ulcerated.

3. Well or moderately differentiated submucosal adenocarcinoma, $<3.0 \mathrm{~cm}$ in size, with superficial submucosal invasion $(\mathrm{Sm} 1 ;<500 \mu \mathrm{m}$ submucosal invasion as measured in a straight line from the deepest fibre of the muscularis mucosae).

4. Poorly differentiated intramucosal adenocarcinoma, $<2.0 \mathrm{~cm}$ in size.

This led to the adoption of the expanded indications for ESD of early gastric cancer. The expanded criteria, however, should not be taken as absolute, particularly where the balance between risk and benefit of surgery is less clear. The original definition of early gastric cancer was defined in 1971 by the Japanese Society of Gastroenterology and Endoscopy as a carcinoma limited to the mucosa and/or submucosa regardless of the lymph node status. This has recently fuelled much controversy as the survival of early gastric cancer is closely associated with the risk of LNM. We have therefore adopted the definition of adenocarcinoma limited to the mucosa and superficial submu$\operatorname{cosa}(\mathrm{Sm} 1 ;<500 \mu \mathrm{m})$, as that amenable to endoscopic resection.

Two recent series, one from the Far East and one from the West, assessed the outcomes of the extended indications. The series by Hasuike et al included 470 lesions, of which 466 (99.1\%) were resected en bloc and the curative resection rate was $67.4 \% .{ }^{218} \mathrm{~A}$ similar Western series reported that en bloc resection was achieved in 81 of 91 lesions (89.0\%) and curative resection was achieved in 67 of 91 lesions $(73.6 \%) .{ }^{219}$ The risk of perforation in both series was reported to be up to $2.6 \%$. However, the risk of delayed bleeding was lower in the Western series (2.2\% compared with 6.2\%). The higher curative resection and lower delayed bleeding rate in the Western series are encouraging, although the number of treated lesions was smaller.

Patient selection is key to achieving favourable outcomes with ESD for early gastric cancer. Patients who do not meet the expanded criteria for a curative outcome following gastric ESD are referred for radical surgery. In a multicentre retrospective study, Hatta et al developed a risk scoring system using multivariate logistic regression analysis of 1101 patients who had undergone radical surgery after failing to meet the criteria for curative endoscopic resection of early gastric cancer. ${ }^{220}$ They then validated the scoring system in a further 905 patients. They showed that the scoring system known as 'eCura system' (table 5) predicted cancer-specific survival in this cohort of patients. This scoring system is promising but will require further validation in other centres.

EMR (cap-assisted) was the initial technique used to resect superficial gastric neoplasia. However, this technique is unable to effectively resect lesions larger than $10 \mathrm{~mm}$ en bloc. In a recent meta-analysis comparing the efficacy of gastric ESD and EMR, the en bloc and R0 resection rates of EMR were found to be $51.7 \%$ and $42.4 \%$, respectively. ${ }^{221}$ This, in turn, is associated with local recurrence rates as high as 30\%. ${ }^{180} 203207222-225$ In a subgroup analysis of lesions smaller than $10-15 \mathrm{~mm}$, it was noted that there was no difference in survival regardless of the endoscopic resection technique.

ESD was a technique developed to overcome the shortcomings of gastric EMR, enabling the en bloc resection of lesions $>10 \mathrm{~mm}$. In a large series from the Far East of 1033 early gastric cancer lesions, Oda et al reported an en bloc resection and R0 resection rate of $98 \%$ and $93 \%$, respectively. ${ }^{226}$ Three meta-analyses comparing the outcomes of EMR and ESD showed that ESD achieved higher en bloc resection rates $(92 \%$ vs $52 \%$; OR $=9.69$, 95\% CI 7.74 to 12.13 ), histopathologically complete resection rates $(82 \%$ vs $42 \%$; OR $=5.66,95 \% \mathrm{CI} 2.92$ to 10.96$)$ and lower recurrence rates $(1 \%$ vs $6 \%$; OR $=0.10,95 \% \mathrm{CI} 0.06$ to 0.18). 221227228

The current Japanese Gastroenterological Endoscopy Society and ESGE guidelines recommend ESD as the preferred treatment for most superficial gastric neoplastic lesions. ${ }^{211} 229$ However, ESD is a technique that is in its infancy in the West, and the complication rates during early adoption can be high. In one European series of 75 patients who underwent gastric ESD, en bloc resection was achieved in $85.3 \%$ and R0 resection in $84.0 \%$. However, the complication rate which included delayed bleeding and perforation was as high as $24 \% \cdot{ }^{230}$ Finally, it should be noted that signet ring cancer is not currently recommended for endoscopic resection. However, prospective data will soon be published on the treatment of these lesions with ESD if they are $<20 \mathrm{~mm}$, which may alter this management strategy.

In summary we recommend, where appropriate, endoscopic resection as first-line treatment for all early gastric neoplasia in line with the Japanese extended indications, favouring ESD over EMR for larger lesions owing to the superior R0 resection rate. Surgery should be undertaken only when endoscopic resection is not considered curative or is the preferred patient option.

\section{Treatment: pharmacological}

Is there a role for other pharmacological therapies for example, COX inhibitors and antioxidants?

We do not recommend the use of NSAIDs or COX-2 inbibitors to reduce the risk of progression of premalignant lesions of the stomach (evidence level: moderate; grade of recommendation: strong; level of agreement: 100\%).

We do not recommend the use of antioxidants as a means to reduce the prevalence of premalignant gastric lesions (evidence level: moderate; grade of recommendation: strong; level of agreement: 100\%). 
NSAIDs, aspirin and COX-2 inhibitors

There are numerous examples of chronic inflammatory conditions that predispose to cancer development. Meta-analyses and retrospective studies have demonstrated that there is a lower incidence of gastric cancer associated with regular, long-term use of NSAIDs, including aspirin. ${ }^{231-233}$ These are thought to reduce carcinogenesis by inhibiting cancer-associated prostaglandins, cytokines and angiogenic factors. However, their use has been limited by their other GI side effects, and there are few goodquality, prospective trials to determine whether they can prevent the progression of premalignant gastric lesions.

Thus, attention has turned to COX-2 inhibitors, with trials particularly focusing on the use of rofecoxib, etodolac and celecoxib. However, there is limited evidence to support their use because of low-quality studies performed in heterogeneous populations in countries with a high incidence of gastric cancer. One placebo-controlled randomised control trial has been carried out, which explored the use of rofecoxib in reversing GIM in those cleared of $H$. pylori. It showed that there was no evidence that rofecoxib causes a regression in GIM over a 2-year period. ${ }^{234}$ Among the low-quality trials, a study using etodolac for patients who underwent endoscopic early cancer resection found a higher rate of metachronous cancer lesions in those treated with the COX-2 inhibitor. ${ }^{235}$ Only celecoxib has been suggested to provoke regression of gastric premalignant lesions; however, in general, these trials are limited to small patient numbers over short periods of time. ${ }^{236-238}$ A large Swedish population-based, case-control study comparing 567 incident cases of gastric cancer and 1165 controls found that aspirin users had a moderately reduced risk of cardia and non-cardia gastric cancer $(\mathrm{OR}=0.7 ; 95 \% \mathrm{CI} 0.6$ to 1.0$)$, although NSAID use had no protective effect in this study. ${ }^{239}$ A recent literature review of 24 studies suggested that both aspirin and NSAIDs reduce the risk of gastric cancer with an RR of 0.7 (95\% CI 0.62 to 0.80$)$ and 0.86 (95\% CI 0.80 to 0.94 ), respectively. ${ }^{240}$ The dose-response analysis indicated that the risk decreased by $11 \%$ and $5 \%$ for 2 years after a dose increment for both.

Further work is undoubtedly required to study the potential of celecoxib, aspirin and NSAIDs in the chemoprevention of premalignant gastric lesions-in particular, in well-designed, randomised, long-term follow-up trials in populations who have a low incidence of gastric cancer. Any benefit must be weighed against the known GI side effects of aspirin and NSAIDs.

\section{Antioxidants}

A limited number of trials have explored the use of antioxidants for chemoprevention of gastric premalignant lesions. Three studies have been performed, all in populations with a high incidence of gastric cancer and with generally disappointing results. Correa et al demonstrated some benefit for histopathological outcome at 6 years following ascorbic acid and $\beta$-carotene use, but this improvement was lost after 12 years. ${ }^{241}$ The other two studies did not show any benefit of using antioxidants in preventing the progression or causing regression of premalignant gastric lesions. ${ }^{242} 243$

\section{Endoscopic screening for gastric adenocarcinoma}

Is there evidence to support the introduction of a population screening programme for glandular gastric cancer?

We suggest endoscopic screening should be considered in individuals aged $\geq 50$ years with multiple risk factors for gastric adenocarcinoma (male, smokers, pernicious anaemia)—in particular, in those with a first-degree relative with gastric cancer (evidence level: low quality; grade of recommendation: weak; level of agreement: $100 \%)$.

We do not recommend endoscopic screening for gastric adenocarcinoma in the UK population (evidence level: low quality; grade of recommendation: strong; level of agreement: 100\%).

Evidence for the effectiveness of endoscopic screening for prevention of gastric cancer has been gathered from studies conducted in high-risk populations (defined as an ASR $>20$ per 100 000); in Japan and Korea (29.9 and 41.3, respectively). ${ }^{244}$ These include five cohort studies and three case-control studies. Although there are no randomised control trials, the results of the available studies suggest a reduction in mortality from gastric cancer in screened populations. In the more recent regional Japanese cohort studies, there was a reduction in gastric cancer mortality (calculated as the standardised mortality ratio or adjusted RR) of $57 \%$ and $67 \%$ after a 5-year and 6-year follow-up. ${ }^{245-247}$ The earlier cohort studies from Japan and China were less convincing but were limited by a broad age distribution and poorly matched cohorts. ${ }^{248} 249$ The results of the case-control studies were equally variable with a reduction in gastric cancer mortality of between $20 \%$ and $80 \%$ in highrisk populations in Japan and South Korea. ${ }^{250} 251$ Based on these results, the authors recommended endoscopic screening in regions with a high incidence of gastric cancer.

The test characteristics of endoscopic screening have been described in four studies. Hosokawa et al found the sensitivity of endoscopy to be $78 \%$ after comparing the gastric cancer incidence in those screened from a cancer registry. ${ }^{252}$ Similar sensitivities of $69 \%$ and $89 \%$ have been found across other cancer registries in Japan and South Korea. ${ }^{253-255}$ In the South Korean study the gastric cancer detection rate was 2.61 per 1000 screening endoscopies, with a specificity of $96 \% .^{253}$

There have been two studies in low-incidence regions (defined as an ASR <10 per 100 000), such as the USA (3.9 per 100 000), assessing the cost-effectiveness of endoscopic screening. The cost of a single-screening endoscopy at the age of 50 in the general population was US\$115 664 per QALY, suggesting that endoscopic screening was not cost-effective. ${ }^{184256}$ Cost-effectiveness analyses in higher risk areas have demonstrated an ICER of US\$44098 and US\$25949 per QALY for annual and biannual screening endoscopy, respectively. ${ }^{186}$ In a study in Taiwan where patients at the age of 50 who had low levels of pepsinogen-I $(<30 \mathrm{ng} / \mathrm{mL})$ were offered endoscopy, the ICER was US\$29741 per life-year gained. ${ }^{257}$

A Markov model of screening in an intermediate-risk population (ASR of $>10$ and $<20$ per 100000 population) for ages 50-75 years found that upper endoscopy combined with screening colonoscopy (every 10 or 5 years) had an ICER of $€ 15$ 407/QALY and €30 908/QALY, respectively. ${ }^{258}$ Standalone endoscopic screening (every 5 years) had an ICER of $€ 70$ 693/ QALY and pepsinogen screening an ICER of $€ 143$ 344/QALY. This work suggests that endoscopic gastric cancer screening in conjunction with a scheduled colonoscopy may be cost-effective in countries with intermediate gastric adenocarcinoma risk such as in Eastern Europe or Portugal. These results imply that resources allocated to endoscopic colorectal cancer screening programmes could be used to provide gastric cancer screening, both for detection of high-risk individuals with extensive premalignant conditions and patients with early gastric cancer.

Although there is insufficient evidence to support screening in low-risk populations, a recent study by Shawihdi and colleagues demonstrated wide variations in rates of elective gastroscopy within general practice populations. They showed that patients with oesophagogastric cancer belonging to practices with low 
rates of gastroscopy were at increased risk of poor outcome. However, if the low referral practices increased to the mid-referral range, the crude cost per life-year saved for a hypothetical scenario is $£ 140000$, well above a suggested threshold of $£ 25000$. Therefore despite the poorer outcomes, given the high costs, primary care physicians should follow a restrictive referral practice. $^{259}$

A recent retrospective nationwide Taiwanese propensity-matched cohort study evaluating the impact of non-screening gastroscopy on gastric cancer-related mortality found that patients with gastric cancer who had undergone gastroscopy in the 5 years before the diagnosis of gastric cancer had a better survival than patients who had never undergone a gastroscopy or whose last gastroscopy was more than 5 years before the diagnosis. The authors found that gastric cancer was detected at an earlier stage in patients who had recently undergone endoscopy. The risk of gastric cancer in this study population is low to moderate. This is the first study showing a significant survival advantage of recent endoscopy in patients with gastric cancer in a region of low to moderate gastric cancer incidence. $^{260}$

There are factors apart from the premalignant stomach that confer a greater risk of gastric adenocarcinoma as described in the 'Risk factors for gastric adenocarcinoma' section. These include family history, particularly those with first-degree relatives, pernicious anaemia with an annual incidence of $0.27 \%$ in unsubstantiated cases, older age, male and smoking. Ethnicity is also related to an increased risk, but this may be due to a higher prevalence of $H$. pylori. In individuals within low-risk populations who have additional risk factors as described above, screening endoscopy may be of value.

In summary, we suggest that only those with multiple risk factors for gastric cancer are considered for screening gastroscopy from the age of 50 . If the gastroscopy results are normal, then we would not recommend any further screening. Where CAG is diagnosed, this guideline should be followed.

\section{Diagnosis and management of epithelial gastric polyps}

What are epithelial gastric polyps and how should they be managed?

We recommend that the number of gastric polyps (or estimated number), location of polyps and size of largest polyp should be clearly documented (evidence level: low quality; grade of recommendation: strong; level of agreement: 100\%).

We recommend that gastric polyps other than FGPs should be biopsied for histopathological assessment (evidence level: low quality; grade of recommendation: strong; level of agreement: $100 \%)$

We recommend that photographic documentation should be undertaken for all polyps or representative polyps, if numerous (evidence level: low quality; grade of recommendation: strong; level of agreement: 100\%).

We recommend that if adenomas or hyperplastic polyps are present, the background mucosa should be endoscopically assessed for GA, GIM, H. pylori and synchronous neoplasia (evidence level: moderate quality; grade of recommendation: strong; level of agreement: 100\%).

We recommend that all adenomas should be resected when clinically appropriate and safe to do so (evidence level: low quality; grade of recommendation: strong; level of agreement: 100\%).

We recommend that a follow-up gastroscopy should be performed at 12 months after complete endoscopic excision of adenomas, then ongoing surveillance gastroscopy annually

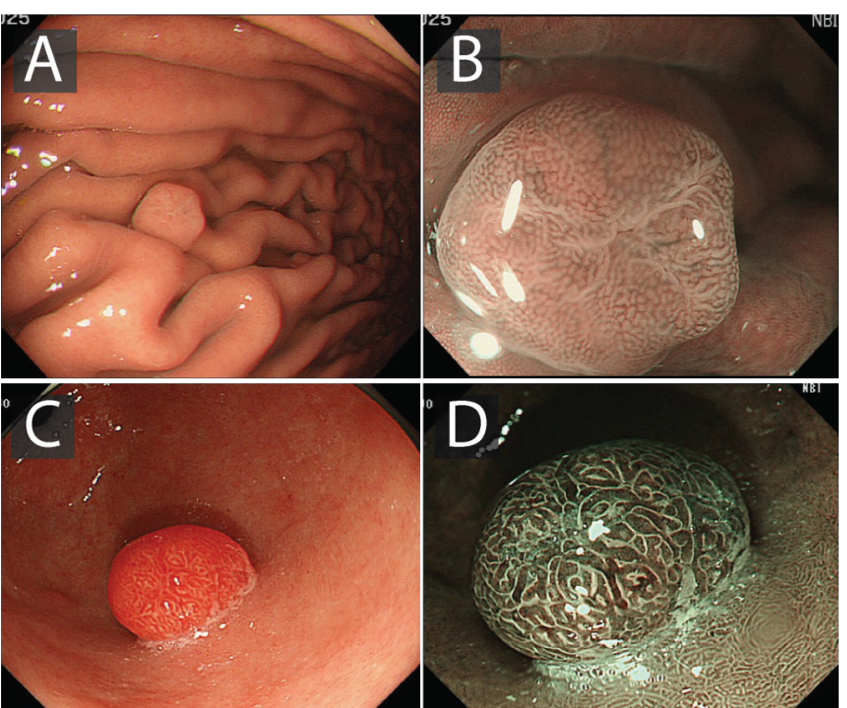

Figure 7 Fundic glandular and hyperplastic polyps. A) Fundic glandular polyps seen in the corpus and body. They are either lighter or the same colour as the surrounding mucosa. B) On near view, with image enhancement, lacy blood vessels are seen through the translucent surface and the surface shows a pattern of fine grey dots. C) Hyperplastic polyps are smooth, red buttered with whitish exudates (fibrin) and are dome shaped. The surface vascular pattern is more prominent on image enhancement (D).

thereafter, when appropriate (evidence level: low quality; grade of recommendation: strong; level of agreement: 93\%).

We suggest that hyperplastic polyps $>1 \mathrm{~cm}$, pedunculated morphology and those causing symptoms (obstruction, bleeding) should be resected. If present, $H$. pylori should be eradicated before re-evaluation for endoscopic therapy (evidence level: low quality; grade of recommendation: weak; level of agreement: $100 \%)$.

We suggest that enhanced endoscopic imaging is used to aid characterisation of gastric polyps when there is diagnostic uncertainty following white light examination (evidence level: low quality; grade of recommendation: weak; level of agreement: 93\%).

The scope of the guidelines is restricted to epithelial polyps, and thus neuroendocrine tumours and subepithelial polyps have been excluded. Gastric epithelial polyps can be mainly classified as three types ${ }^{107}$ : FGPs, hyperplastic polyps and adenomatous polyps.

\section{Fundic gland polyps}

FGPs are the most prevalent type of gastric polyps (13-77\%). ${ }^{261262}$ They are typically multiple, small $(<1 \mathrm{~cm})$ and located in the fundus and corpus. At endoscopy they appear pale, smooth, glassy, and transparent or translucent (figure 7). Their colour is either lighter or the same colour as the surrounding mucosa. Lacy blood vessels are seen through the translucent surface and the surface shows a pattern of fine grey dots. On enhanced imaging such as NBI, FICE or i-Scan, the surface architecture becomes more prominent. FGPs are usually not associated with an increased risk of cancer, unless in the context of FAP syndrome. However, larger FGPs $(>1 \mathrm{~cm})$ have been shown to be dysplastic in $1.9 \%$ and contain focal cancer also in $1.9 \%$. FGPs are associated with long-term PPI use and can spontaneously regress when PPIs are stopped. ${ }^{263}$ There is no association with background $H$. pylori infection or gastritis. 


\section{Management of FGPS}

Number, location, morphology and the size of the largest polyp should be documented. Representative pictures of the polyps should be taken. Diagnosis is easily made from the endoscopic appearance, as described above, but biopsy confirmation should be sought when in doubt. Large numbers of polyps $(>20)$, young age ( $<40$ years), dysplastic appearing polyps (where the typical surface and vascular architecture alter particularly when irregular) and the presence of duodenal adenomas should lead to exclusion of FAP. ${ }^{107}$ FGPs do not require excision unless they have atypical features. Size of $>1 \mathrm{~cm}$, antral location, ulceration or an unusual appearance should question the diagnosis of FGP and lead to excision. ${ }^{264}$ Targeted biopsies should be taken where excision is not undertaken. Patients receiving long-term PPIs should be re-evaluated for appropriateness of the PPI, dose of PPI and alternative treatments. ${ }^{263}$ There is no role for surveillance gastroscopy for FGPs, except in the setting of FAP. ${ }^{107}$

\section{Hyperplastic polyps}

Hyperplastic polyps constitute $18-70 \%$ of all gastric polyps, are usually single or few in number and are more frequently observed in the antrum or adjacent to ulcers, stomas and gastrectomy sites. They appear as smooth, red buttered with whitish exudates (fibrin) and are dome-shaped (figure 7). They are usually small $(0.5-1.5 \mathrm{~cm})$, but may be larger and present as lobulated and pedunculated masses covered with superficial erosions. They are typically associated with $H$. pylori gastritis (25\%), GA and GIM. Regression generally occurs after eradication of $H$. pylori (up to $70 \%){ }^{108}$

Gastric hyperplastic polyps can reveal dysplasia (1.9-19\%) and malignant transformation $(0.6-2.1 \%),{ }^{265-267}$ especially when $>1 \mathrm{~cm}$ and in the postgastrectomy stomach. ${ }^{267-270} \mathrm{~A}$ dysplastic hyperplastic polyp is associated with an increased risk of synchronous neoplastic lesions in the surrounding mucosa of approximately $6 \%$ of cases. ${ }^{271-277}$

Small, white and flat plaques in the fundus have the appearance of hyperplastic polyps with a foveolar pit pattern, but are areas of focal foveolar hyperplasia or more specifically, hyperplasia of the foveolar epithelium. ${ }^{278}$ These have been described as multiple white flat lesions (MWFLs) and appear to be more prevalent in those taking PPIs. Histologically, the biopsy specimens from the MWFLs included fundic gland parietal cell protrusions and oxyntic gland dilatations. There was no evidence of intestinal metaplasia. ${ }^{279}$

\section{Management of hyperplastic polyps}

Diagnosis of hyperplastic polyps and the absence of dysplasia should be confirmed by histology. The remainder of the stomach should be carefully evaluated for synchronous neoplasia, degree and extent of GA and H. pylori. H. pylori eradication should be considered in all cases before endoscopic resection as many polyps will regress, and a repeat endoscopy carried out 3-6 months after eradication. ${ }^{108}$ Polyps of $>1 \mathrm{~cm}$, pedunculated polyp morphology or symptomatic polyps (obstruction, bleeding) should be completely resected. ${ }^{268} 280$ Even when $H$. pylori is present, those polyps $>3 \mathrm{~cm}$ should always be resected as the risk of dysplasia and cancer is high. ${ }^{267} 280$ Endoscopic surveillance is recommended to monitor the risk of further gastric neoplasia where there is evidence of dysplasia, GA or GIM. The endoscopic surveillance interval should be determined by the stage of CAG.

\section{Adenomatous polyps}

Adenomatous polyps are usually single $(82 \%)$, small $(<2 \mathrm{~cm})$ and located in the antrum and incisura angularis. Endoscopically they have a velvety pink lobulated appearance and can be sessile or pedunculated. In Western countries their prevalence varies between $0.5 \%$ and $10 \% .^{264281}$ They are normally associated with a background of GA and GIM. Coexistence of a synchronous gastric adenocarcinoma has been found in up to $30 \%$ of patients with an adenomatous polyp. Moreover, 50\% of adenomatous polyps $>2 \mathrm{~cm}$ contain foci of adenocarcinoma. ${ }^{196282}$

\section{Management of gastric adenomas}

Gastric adenomas carry a significant risk of progression to cancer and should be resected where appropriate. Diagnosis of adenoma and degree of dysplasia should be histologically confirmed before treatment is undertaken. A careful evaluation of the stomach should be carried out to identify synchronous neoplasia (30\%), GA and GIM. Endoscopic resection is the preferred mode of treatment. An en bloc excision with ESD is advisable for sessile polyps $>15 \mathrm{~mm}^{211}$ as the possibility of invasive neoplasia in the adenoma is high and ESD reduces the risk of recurrence compared with EMR. A follow-up gastroscopy should be performed at $6-12$ months after endoscopic resection of adenomas. Patients with adenomas should continue to have surveillance gastroscopy at yearly intervals where appropriate, depending on the number of polyps, their size and the highest grade of dysplasia.

\section{Optical diagnosis of gastric polyps}

Optical enhancement with technology, including NBI (Olympus), i-Scan (Pentax) and FICE (Fujinon) with or without near focus or magnification allows interpretation of the mucosal surface architectural patterns and vasculature. FGPs are characterised by small round pits and a honeycomb microvascular pattern (sensitivity 94.7\%, specificity 97.4\%), whereas hyperplastic polyps display prolonged or villous pits and a dense vascular pattern (sensitivity 93.6\%, specificity 91.6\%). ${ }^{283}$ The NBI features of gastric adenomas have not been well defined, although a paler colour relative to the background mucosa and a slit-like crypt opening and regular white opaque substance, which corresponds to absorbed subepithelial lipid droplets, are often observed. The brown colour on NBI is due to the subepithelial vessels, which are obscured by lipid droplets accumulated above the subepithelial vessels. A tubular, villous or ridged mucosal structure is generally observed as with colonic adenomas. ${ }^{153} 284285$

Based on the National Institute for Health and Care Excellence classification validated for colon polyps, a modified NBI classification system has been recently proposed in a retrospective evaluation by two expert reviewers to reliably diagnose low-risk gastric polyps (FGPs or hyperplastic polyps $<1 \mathrm{~cm}$ ) with the aim of eliminating the need for biopsy. ${ }^{286}$

Relative to the background mucosa, polyps were divided in two groups:

1. Lighter or same colour.

2. Darker.

'Lighter or same colour' polyps were further subdivided according to their vascular network in:

a. Isolated vessels.

b. No vessels.

c. Brown vessels surrounding white structures.

Polyps with same or lighter colour and with no vessels or isolated lacy vessels were FGPs in 97-100\%. Polyps lighter or similar in colour to the background mucosa with a homogeneous 
absence of surface pattern were FGPs in 94-100\%. The sensitivity and specificity for lacy vessels were $61-66 \%$ and $93-96 \%$, respectively, for optical FGP diagnosis. If a lesion had either isolated lacy vessels or homogeneous absence of pattern, the negative predictive value for gastric adenoma or cancer was $100 \%$. The negative predictive value for colour the same or lighter than the background mucosa was 96-97\%. Polyps with colour lighter or the same as the background mucosa, but $>1 \mathrm{~cm}$ or showing brown vessels surrounding white structures, or those darker in colour, require biopsy. Although useful as a research algorithm for assisting decision-making, further studies and validation of this classification are required.

All polyps where the mucosal pattern is disorganised, irregular or absent (amorphous) are suspicious for neoplasia. Likewise, enhanced or irregular vascular patterns are suspicious for neoplasia, and targeted biopsies should be carried out.

In summary, IEE improves the visualisation of gastric polyps and allows the diagnosis of FGPs. Diagnosis of hyperplastic polyps and adenomas with IEE is less clear, but may aid in the diagnosis of HGD or cancer. We therefore have suggested that IEE is used in all polyps to aid diagnosis and direct biopsies.

It is important to emphasise that the evidence for the detection, characterisation and management of gastric polyps was generated using lower resolution endoscopes, and thus further studies are needed to investigate the accuracy of optical and targeted histological diagnosis with newer endoscopes. Currently, the evidence supports endoscopic resection rather than biopsy of many non-fundic glandular polyps given the likelihood of upstaging neoplasia. ${ }^{287}$ This is of particular importance given the risks of bleeding associated with gastric polypectomy.

\section{EDUCATION, AUDIT, AND BENCHMARKS AND RESEARCH QUESTIONS}

\section{Audit and benchmarks}

The prevalence of GIM in patients undergoing endoscopy for dyspepsia is as high as $25 \%$ in European studies. The prevalence of GIM is influenced by ethnicity, infection rates with $H$. pylori, age and family history of gastric cancer. GIM is present in $100 \%$ of intestinal-type gastric cancer. Detection of GIM is therefore crucial as a first step in order to identify those at risk of gastric adenocarcinoma and may be an obvious benchmark as a quality standard for upper GI endoscopy. The prevalence of GA in Western populations is lower than GIM and varies from $0 \%$ to $8 \%$, as previously described. We suggest a benchmark of $10 \%$ detection rate for GIM and/or GA in those patients undergoing investigation for upper GI symptoms.

\section{Education}

The miss rate for gastric cancer on endoscopy is high, and awareness of the endoscopic features of its precursors, GA and GIM, is low. We suggest that knowledge of these pathologies is incorporated into the new national gastroenterology curriculum for higher training for gastroenterologists, surgeons and pathologists.

\section{Service and cost implications of the guidelines}

An extensive service evaluation has not been conducted for this guideline. We have made an estimate of the likely additional work generated by their adoption. As discussed, in Western populations (Europe and USA) the overall prevalence of CAG in young men and women ( $<55$ years) was $0-8.3 \%$. In older age groups ( $>55$ years), the prevalence was reported to be up to $13 \%{ }^{20}$ and in the EUROGAST study $<5 \%{ }^{19}$ and $5.3 \%$ in those aged 55-64 years.
Of the 1.7 million endoscopies performed each year in the UK, approximately $40 \%$ are upper GI procedures. ${ }^{288}$ Thus, in a unit performing a total of 10000 procedures a year, 4000 will be upper GI endoscopies, of which approximately 200 patients will have CAG. Assuming endoscopic diagnostic accuracy of 100\% and a CAG prevalence of 5\%, this will give rise to 200 additional sets of Sydney biopsies, assuming the unit was not previously taking biopsy samples for CAG. This is likely to result in a repeat procedure in a proportion of cases where IEE was not available during the initial endoscopy. There are no clear data to define the proportion of CAG that extensively affects the stomach and therefore requires surveillance, but we have estimated this to be $40 \%$ of all CAG diagnosed based on our own experience. We estimate that there will be 130 patients per unit requiring surveillance endoscopy between 1 and 3 yearly.

\section{Future research}

This guideline aims to improve the standardisation of practice in the management of patients at risk of gastric adenocarcinoma. We envisage that improved endoscopic quality, and consequently the detection of CAG and early gastric neoplasia, with targeted surveillance will improve the outcomes of gastric cancer. However, the effect of the guidelines on gastric cancer survival nationally is likely to be small, principally because only a small proportion of patients with gastric cancer will be detected by endoscopy at an early stage. Therefore, studies of cost-effective, non-invasive, population-based screening should be a research priority of the next 5-7years. The other main priority is a measurable improvement in the quality of gastroscopy.

Quality standards (QA) and improvement (QI)

- We suggest that a QI bundle for upper GI endoscopy requires derivation and assessment as a priority for research in order to improve diagnostic rates of early gastric neoplasia and its precursors (GA and GIM).

- We suggest that quality indicators are required for systematic gastric surveillance endoscopy and photographic documentation.

Screening and surveillance

- We suggest pilot studies for non-invasive, population-based screening strategies for gastric cancer are a research priority.

- We suggest that a pilot study is required to assess the cost-effectiveness of endoscopic gastric cancer screening when combined with a screening colonoscopy.

- We suggest that further research is required to investigate the optimal surveillance strategy for CAG,

Diagnosis and staging

- We suggest that the accuracy and reproducibility of optical diagnosis and staging of GA and GIM need to be investigated in a multicentre study.

- We suggest research to determine whether the severity and distribution of CAG categorised by OLGA and OLGIM accurately reflect cancer risk during follow-up.

- Cytosponge has been shown to detect cardia intestinal metaplasia, ${ }^{289}$ but more research is needed to see if this might be a tool to help identify cardia IM as a triage to endoscopy.

- We suggest that further research is required to investigate the risk of progression of histologically and serologically confirmed pernicious anaemia.

- We suggest that the natural history and risk of progression to cancer of visible and non-visible LGD requires further research.

Prevalence 
- We suggest that further research is required to quantify more accurately the prevalence and extent of GIM in European endoscopy practice.

\section{Planned review date}

The guidelines should be considered for review in 5 years from the date of submission for publication, estimated to be November 2023.

\author{
Author affiliations \\ ${ }^{1}$ University College London Hospital, University College London Hospitals NHS \\ Foundation Trust, London, UK \\ ${ }^{2}$ Research Department of Targeted Intervention, University College London, London, \\ UK \\ ${ }^{3}$ Division of Surgery and Interventional Science, University College London Division of \\ Biosciences, London, UK \\ ${ }^{4}$ Department of Histopathology, University College London, London, UK \\ ${ }^{5}$ Gastroenterology, Nihon University School of Medicine Graduate School of \\ Medicine, Itabashi-ku, Tokyo, Japan \\ ${ }^{6}$ Endoscopy, Inhealth Group, London, UK \\ ${ }^{7}$ MRC Cancer Unit, University of Cambridge, Cambridge, UK \\ ${ }^{8}$ Gastroenterology, Cambridge University Hospitals NHS Foundation Trust, \\ Cambridge, UK \\ ${ }^{9}$ Department of Gastrointestinal Oncology, Endoscopic Training and Learning \\ Center, Osaka Medical Center for Cancer and Cardiovascular Diseases, Osaka, \\ Japan \\ ${ }^{10}$ Gastroenterology, Portsmouth, Portsmouth, UK \\ ${ }^{11}$ Institute of Translational Medicine, University of Liverpool, Liverpool, UK \\ ${ }^{12}$ Erasmus University Medical Center, Rotterdam, Netherlands \\ ${ }^{13}$ Nottingham Digestive Diseases Centre, Nottingham University Hospital, \\ Nottingham, UK \\ ${ }^{14}$ Gloucestershire Cellular Pathology Laboratory, Cheltenham General Hospital, \\ Cheltenham, Gloucestershire, UK \\ ${ }^{15}$ Gastroenterology, IPO Porto, Porto, Portugal
}

Acknowledgements We acknowledge the BSG for support in the development of these guidelines. We thank Dr Marc Tischkowitz for his contribution to the genetics of gastric cancer, Reader and Honorary Consultant in Medical Genetics, Academic Department of Medical Genetics, NIHR Cambridge Biomedical Research Centre, University of Cambridge, Box 238, Level 6 Addenbrooke's Treatment Centre, Cambridge Biomedical Campus, Cambridge CB2 0QQ.

Collaborators Marc Tischkowitz.

Contributors Each coauthor's contribution was dependent upon their role in the subgroups and varied across the guideline development group.

Funding The authors have not declared a specific grant for this research from any funding agency in the public, commercial or not-for-profit sectors. This guidelines was supported by the BSG for meetings only

Competing interests None declared.

Patient consent for publication Not required.

Provenance and peer review Not commissioned; externally peer reviewed.

Open access This is an open access article distributed in accordance with the Creative Commons Attribution Non Commercial (CC BY-NC 4.0) license, which permits others to distribute, remix, adapt, build upon this work non-commercially, and license their derivative works on different terms, provided the original work is properly cited, appropriate credit is given, any changes made indicated, and the use is non-commercial. See: http://creativecommons.org/licenses/by-nc/4.0/.

\section{REFERENCES}

1 Amiri M, Janssen F, Kunst AE. The decline in stomach cancer mortality: exploration of future trends in seven European countries. Eur J Epidemiol 2011;26:23-8.

2 Rahman R, Asombang AW, Ibdah JA. Characteristics of gastric cancer in Asia. World J Gastroenterol 2014:20:4483-90.

3 Cancer Research UK. Stomach cancer statistics | Cancer Research UK [Internet].

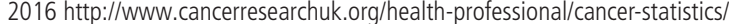
statistics-by-cancer-type/stomach-cancer (cited 14 Mar 2018).

4 Song $\mathrm{H}$, Held M, Sandin S, et al. Increase in the prevalence of atrophic gastritis among adults age 35 to 44 years old in northern Sweden between 1990 and 2009. Clin Gastroenterol Hepatol 2015:13:1592-600.

5 Anderson WF, Camargo MC, Fraumeni JF, et al. Age-specific trends in incidence of noncardia gastric cancer in US adults. JAMA 2010;303:1723-8.

6 Maconi G, Manes G, Porro GB. Role of symptoms in diagnosis and outcome of gastric cancer. World J Gastroenterol 2008;14:1149-55.
7 Matsuda T, Saika K. The 5-year relative survival rate of stomach cancer in the USA, Europe and Japan. Jpn J Clin Oncol 2013;43:1157-8.

8 Correa P, Haenszel W, Cuello C, et al. A model for gastric cancer epidemiology. Lancet 1975;2:58-60.

9 Correa P. A human model of gastric carcinogenesis. Cancer Res 1988;48:3554-60.

10 Dinis-Ribeiro M, Areia M, de Vries AC, et al. Management of precancerous conditions and lesions in the stomach (MAPS): guideline from the European Society of Gastrointestinal Endoscopy (ESGE), European Helicobacter Study Group (EHSG), European Society of Pathology (ESP), and the Sociedade Portuguesa de Endoscopia Digestiva (SPED). Endoscopy 2012;44:74-94.

11 Tham TC, Gleeson D, Greenfield SM, et al. British Society of Gastroenterology policy and processes for the development of guidelines. Gut 2015;64:1184-5.

12 Appraisal of guidelines for research \& evaluation II. The AGREE Research Trust. Update: September 2013. www.agreetrust.org.

13 Spence $A D$, Cardwell CR, McMenamin ÚC, et al. Adenocarcinoma risk in gastric atrophy and intestinal metaplasia: a systematic review. BMC Gastroenterol 2017:17:157.

14 Guyatt GH, Oxman AD, Vist GE, et al. GRADE: an emerging consensus on rating quality of evidence and strength of recommendations. BMJ 2008;336:924-6.

15 Song H, Ekheden IG, Zheng Z, et al. Incidence of gastric cancer among patients with gastric precancerous lesions: observational cohort study in a low risk Western population. BMJ 2015;351:h3867.

16 Miwata T, Quach DT, Hiyama T, et al. Interobserver and intraobserver agreement for gastric mucosa atrophy. BMC Gastroenterol 2015;15:95

17 Leja M, Funka K, Janciauskas D, et al. Interobserver variation in assessment of gastric premalignant lesions: higher agreement for intestinal metaplasia than for atrophy. Eur I Gastroenterol Hepatol 2013;25:694-9.

18 Toyoshima O, Yamaji Y, Yoshida S, et al. Endoscopic gastric atrophy is strongly associated with gastric cancer development after Helicobacter pylori eradication. Surg Endosc 2017;31:2140-8.

19 Webb PM, Hengels KJ, Møller H, et al. The epidemiology of low serum pepsinogen A levels and an international association with gastric cancer rates. EUROGAST Study Group. Gastroenterology 1994;107:1335-44.

20 Weck MN. Prevalence of chronic atrophic gastritis in different parts of the world. Cancer Epidemiol Biomarkers Prev 2006;15:1083-94.

21 Choi CE, Sonnenberg A, Turner K, et al. High prevalence of gastric preneoplastic lesions in East Asians and Hispanics in the USA. Dig Dis Sci 2015:60:2070-6.

22 Namekata T, Miki K, Kimmey M, et al. Chronic atrophic gastritis and Helicobacter pylori infection among Japanese Americans in Seattle. Am J Epidemiol 2000;151:820-30.

23 Adamu MA, Weck MN, Gao L, et al. Incidence of chronic atrophic gastritis: systematic review and meta-analysis of follow-up studies. Eur I Epidemiol 2010;25:439-48.

24 Vannella L, Lahner E, Annibale B. Risk for gastric neoplasias in patients with chronic atrophic gastritis: a critical reappraisal. World I Gastroenterol 2012;18:1279-85.

25 de Vries AC, van Grieken NC, Looman CW, et al. Gastric cancer risk in patients with premalignant gastric lesions: a nationwide cohort study in the Netherlands. Gastroenterology 2008;134:945-52.

26 Shichijo S, Hirata Y, Niikura R, et al. Histologic intestinal metaplasia and endoscopic atrophy are predictors of gastric cancer development after Helicobacter pylori eradication. Gastrointest Endosc 2016;84:618-24.

27 Eriksson NK, Kärkkäinen PA, Färkkilä MA, et al. Prevalence and distribution of gastric intestinal metaplasia and its subtypes. Dig Liver Dis 2008;40:355-60.

28 Olmez S, Aslan M, Erten R, et al. The prevalence of gastric intestinal metaplasia and distribution of Helicobacter pylori infection, atrophy, dysplasia, and cancer in its subtypes. Gastroenterol Res Pract 2015;2015:1-6

29 Eidt S, Stolte M. Prevalence of intestinal metaplasia in Helicobacter pylori gastritis. Scand J Gastroenterol 1994;29:607-10.

30 Tulassay Z, Stolte M, Engstrand L, et al. Twelve-month endoscopic and histological analysis following proton-pump inhibitor-based triple therapy in Helicobacter pyloripositive patients with gastric ulcers. Scand J Gastroenterol 2010;45:1048-58.

31 Uemura N, Okamoto S, Yamamoto S, et al. Helicobacter pylori infection and the development of gastric cancer. N Engl J Med Overseas Ed 2001;345:784-9.

32 Zullo A, Hassan C, Romiti A, et al. Follow-up of intestinal metaplasia in the stomach: when, how and why. World I Gastrointest Oncol 2012;4:30-6.

33 Park YH, Kim N. Review of atrophic gastritis and intestinal metaplasia as a premalignant lesion of gastric cancer. J Cancer Prev 2015;20:25-40.

34 González CA, Sanz-Anquela JM, Companioni 0, et al. Incomplete type of intestina metaplasia has the highest risk to progress to gastric cancer: results of the Spanish follow-up multicenter study. I Gastroenterol Hepatol 2016:31:953-8.

35 Dinis-Ribeiro M, Lopes C, da Costa-Pereira A, et al. A follow up model for patients with atrophic chronic gastritis and intestinal metaplasia. J Clin Pathol 2004;57:177-82

36 Cassaro M, Rugge M, Gutierrez 0 , et al. Topographic patterns of intestinal metaplasia and gastric cancer. Am J Gastroenterol 2000;95:1431-8.

37 Vannella L, Lahner E, Osborn J, et al. Risk factors for progression to gastric neoplastic lesions in patients with atrophic gastritis. Aliment Pharmacol Ther 2010;31:1042-50. 
38 Lahner E, Bordi C, Cattaruzza MS, et al. Long-term follow-up in atrophic body gastritis patients: atrophy and intestinal metaplasia are persistent lesions irrespective of Helicobacter pylori infection. Aliment Pharmacol Ther 2005;22:471-81.

39 Rugge $M$, Fassan M, Pizzi M, et al. Operative link for gastritis assessment vs operative link on intestinal metaplasia assessment. World J Gastroenterol 2011;17:4596-601.

40 Capelle LG, de Vries AC, Haringsma J, et al. The staging of gastritis with the OLGA system by using intestinal metaplasia as an accurate alternative for atrophic gastritis. Gastrointest Endosc 2010;71:1150-8.

41 Weston AP, Krmpotich P, Makdisi WF, et al. Short segment Barrett's esophagus: clinical and histological features, associated endoscopic findings, and association with gastric intestinal metaplasia. Am J Gastroenterol 1996;91:981-6.

42 Johnston MH, Hammond AS, Laskin W, et al. The prevalence and clinical characteristics of short segments of specialized intestinal metaplasia in the distal esophagus on routine endoscopy. Am J Gastroenterol 1996:91:1507-11.

43 Sharma P, Weston AP, Morales T, et al. Relative risk of dysplasia for patients with intestinal metaplasia in the distal oesophagus and in the gastric cardia. Gut 2000;46:9-13.

44 Sung JK. Diagnosis and management of gastric dysplasia. Korean J Intern Med 2016:31:201-9.

45 Lauwers GY, Riddell RH. Gastric epithelial dysplasia. Gut 1999:45:784.

$46 \mathrm{Kim}$ JW, Jang JY. Optimal management of biopsy-proven low-grade gastric dysplasia. World J Gastrointest Endosc 2015;7:396-402.

47 Baek DH, Kim GH, Park DY, et al. Gastric epithelial dysplasia: characteristics and long-term follow-up results after endoscopic resection according to morphological categorization. BMC Gastroenterol 2015;15:17

48 Shin CM, Kim N, Yang HJ, et al. Stomach cancer risk in gastric cancer relatives: interaction between Helicobacter pylori infection and family history of gastric cancer for the risk of stomach cancer. J Clin Gastroenterol 2010;44:e34-9.

49 Lissowska J, Groves FD, Sobin LH, et al. Family history and risk of stomach cancer in Warsaw, Poland. Eur J Cancer Prev 1999;8:223-7.

50 Nagase H, Ogino K, Yoshida I, et al. Family history-related risk of gastric cancer in Japan: a hospital-based case-control study. Jpn J Cancer Res 1996;87:1025-8.

51 Jiang $X$, Tseng CC, Bernstein L, et al. Family history of cancer and gastroesophageal disorders and risk of esophageal and gastric adenocarcinomas: a case-control study. BMC Cancer 2014;14:60.

52 Kwak HW, Choi IJ, Kim CG, et al. Individual having a parent with early-onset gastric cancer may need screening at younger age. World J Gastroenterol 2015;21:4592-8.

53 Reddy KM, Chang JI, Shi JM, et al. Risk of gastric cancer among patients with intestinal metaplasia of the stomach in a US integrated health care system. Clin Gastroenterol Hepatol 2016;14:1420-5.

54 Lichtenstein P, Holm NV, Verkasalo PK, et al. Environmental and heritable factors in the causation of cancer - analyses of cohorts of twins from Sweden, Denmark, and Finland. N Engl J Med Overseas Ed 2000:343:78-85.

55 Oliveira C, Pinheiro H, Figueiredo J, et al. Familial gastric cancer: genetic susceptibility, pathology, and implications for management. Lancet Oncol 2015;16:e60-e70.

56 Rudloff U. Gastric adenocarcinoma and proximal polyposis of the stomach: diagnosis and clinical perspectives. Clin Exp Gastroenterol 2018;11:447-59.

57 Repak R, Kohoutova D, Podhola M, et al. The first European family with gastric adenocarcinoma and proximal polyposis of the stomach: case report and review of the literature. Gastrointest Endosc 2016:84:718-25.

58 Capelle LG, Van Grieken NC, Lingsma HF, et al. Risk and epidemiological time trends of gastric cancer in Lynch syndrome carriers in the Netherlands. Gastroenterology 2010;138:487-92.

59 Heald B, Mester J, Rybicki L, et al. Frequent gastrointestinal polyps and colorectal adenocarcinomas in a prospective series of PTEN mutation carriers. Gastroenterology 2010;139:1927-33.

60 Syngal S, Brand RE, Church JM, et al. ACG clinical guideline: Genetic testing and management of hereditary gastrointestinal cancer syndromes. Am J Gastroenterol 2015; 110:223-62.

61 Kluijt I, Sijmons RH, Hoogerbrugge N, et al. Familial gastric cancer: guidelines for diagnosis, treatment and periodic surveillance. Fam Cancer 2012;11:363-9.

62 van der Post RS, Vogelaar IP, Carneiro F, et al. Hereditary diffuse gastric cancer: updated clinical guidelines with an emphasis on germline $C D H 1$ mutation carriers. J Med Genet 2015:52:361-74

63 Fitzgerald RC, Hardwick R, Huntsman D, et al. Hereditary diffuse gastric cancer: updated consensus guidelines for clinical management and directions for future research. J Med Genet 2010;47:436-44.

64 Persson C, Canedo P, Machado JC, et al. Polymorphisms in inflammatory response genes and their association with gastric cancer: a HuGE systematic review and metaanalyses. Am J Epidemiol 2011;173:259-70.

65 Vannella L, Lahner E, Osborn J, et al. Systematic review: gastric cancer incidence in pernicious anaemia. Aliment Pharmacol Ther 2013:37:375-82.

66 Murphy G, Dawsey SM, Engels EA, et al. Cancer risk after pernicious anemia in the US elderly population. Clin Gastroenterol Hepatol 2015;13:2282-9.

67 Rusak E, Chobot A, Krzywicka A, et al. Anti-parietal cell antibodies - diagnostic significance. Adv Med Sci 2016;61:175-9.
68 Minalyan A, Benhammou JN, Artashesyan A, et al. Autoimmune atrophic gastritis: current perspectives. Clin Exp Gastroenterol 2017;10:19-27.

69 Fisher SG, Davis F, Nelson R, et al. A cohort study of stomach cancer risk in men after gastric surgery for benign disease. J Natl Cancer Inst 1993:85:1303-10.

70 Tersmette AC, Goodman SN, Offerhaus GJ, et al. Multivariate analysis of the risk of stomach cancer after ulcer surgery in an Amsterdam cohort of postgastrectomy patients. Am J Epidemiol 1991;134:14-21.

71 Viste A, Bjørnestad E, Opheim P, et al. Risk of carcinoma following gastric operations for benign disease. A historical cohort study of 3470 patients. Lancet 1986;2:502-5.

72 Schafer LW, Larson DE, Melton LJ, et al. The risk of gastric carcinoma after surgical treatment for benign ulcer disease. A population-based study in Olmsted County, Minnesota. N Engl J Med 1983;309:1210-3.

73 Lagergren J, Lindam A, Mason RM. Gastric stump cancer after distal gastrectomy for benign gastric ulcer in a population-based study. Int J Cancer 2012;131:E104 8-E1052.

74 Jemal A, Siegel R, Ward E, et al. Cancer statistics, 2006. CA Cancer J Clin 2006;56:106-30.

75 Ali R, Barnes I, Cairns BJ, et al. Incidence of gastrointestinal cancers by ethnic group in England, 2001-2007. Gut 2013:62:1692-703.

76 Lui FH, Tuan B, Swenson SL, et al. Ethnic disparities in gastric cancer incidence and survival in the USA: an updated analysis of 1992-2009 SEER data. Dig Dis SC 2014:59:3027-34.

77 Jin $\mathrm{H}$, Pinheiro PS, Xu J, et al. Cancer incidence among Asian American populations in the United States, 2009-2011. Int J Cancer 2016:138:2136-45.

78 Dong E, Duan L, Wu BU. Racial and ethnic minorities at increased risk for gastric cancer in a regional US population study. Clin Gastroenterol Hepatol 2017; 15:511-7

79 Pérez-Rodríguez M, Partida-Rodríguez 0, Camorlinga-Ponce M, et al. Polymorphisms in HLA-DQ genes, together with age, sex, and Helicobacter pylori infection, as potential biomarkers for the early diagnosis of gastric cancer. Helicobacter 2017;22:e12326.

80 Lee TY, Wang RC, Lee YC, et al. The incidence of gastric adenocarcinoma among patients with gastric intestinal metaplasia: a long-term cohort study. J Clin Gastroenterol 2016;50:532-7.

81 Leung WK, Lin SR, Ching JY, et al. Factors predicting progression of gastric intestinal metaplasia: results of a randomised trial on Helicobacter pylori eradication. Gut 2004;53:1244-9.

82 You WC, Li JY, Blot WJ, et al. Evolution of precancerous lesions in a rural Chinese population at high risk of gastric cancer. Int J Cancer 1999:83:615-9.

83 Theuer CP, de Virgilio C, Keese G, et al. Gastric adenocarcinoma in patients 40 years of age or younger. Am J Surg 1996;172:473-7.

84 Engel LS, Chow WH, Vaughan TL, et al. Population attributable risks of esophageal and gastric cancers. J Natl Cancer Inst 2003:95:1404-13.

85 Sjödahl K, Lu Y, Nilsen TI, et al. Smoking and alcohol drinking in relation to risk of gastric cancer: a population-based, prospective cohort study. Int $J$ Cancer 2007:120:128-32.

86 Nomura AMY, Wilkens LR, Henderson BE, et al. The association of cigarette smoking with gastric cancer: the multiethnic cohort study. Cancer Causes Control 2012:23:51-8.

87 Morais S, Rodrigues S, Amorim L, et al. Tobacco smoking and intestinal metaplasia: systematic review and meta-analysis. Dig Liver Dis 2014;46:1031-7.

88 Song $\mathrm{H}$, Zhu J, Lu D. Long-term proton pump inhibitor (PPI) use and the development of gastric pre-malignant lesions. Cochrane Database Syst Rev 2014;12:CD010623.

89 Ge Z, Ben Q, Qian J, et al. Diabetes mellitus and risk of gastric cancer: a systematic review and meta-analysis of observational studies. Eur J Gastroenterol Hepatol 2011;23:1127-35.

90 Tramacere I, Negri E, Pelucchi C, et al. A meta-analysis on alcohol drinking and gastric cancer risk. Ann Oncol 2012;23:28-36.

91 Lin XJ, Wang CP, Liu XD, et al. Body mass index and risk of gastric cancer: a metaanalysis. Jpn J Clin Oncol 2014;44:783-91.

92 Brusselaers N, Wahlin K, Engstrand L, et al. Maintenance therapy with proton pump inhibitors and risk of gastric cancer: a nationwide population-based cohort study in Sweden. BMJ Open 2017;7:e017739.

93 Cheung KS, Chan EW, Wong AYS, et al. Long-term proton pump inhibitors and risk of gastric cancer development after treatment for Helicobacter pylori: a populationbased study. Gut 2018:67:28-35.

94 Schneider JL, Kolitsopoulos F, Corley DA. Risk of gastric cancer, gastrointestinal cancers and other cancers: a comparison of treatment with pantoprazole and other proton pump inhibitors. Aliment Pharmacol Ther 2016;43:73-82.

95 BSG Position Statement - Proton pump inhibitors (PPIs) and the risk of gastric cancer [Internet]. https://www.bsg.org.uk/resource/bsg-position-statement-proton-pumpinhibitors-ppis-and-the-risk-of-gastric-cancer.html (cited 27 Jan 2019).

96 Cai X, Carlson J, Stoicov C, et al. Helicobacter felis eradication restores normal architecture and inhibits gastric cancer progression in C57BL/6 mice. Gastroenterology 2005; 128:1937-52

97 Lee CW, Rickman B, Rogers AB, et al. Helicobacter pylori eradication prevents progression of gastric cancer in hypergastrinemic INS-GAS mice. Cancer Res 2008;68:3540-8 
98 Ford AC, Forman D, Hunt RH, et al. Helicobacter pylori eradication therapy to prevent gastric cancer in healthy asymptomatic infected individuals: systematic review and meta-analysis of randomised controlled trials. BMJ 2014;348:g3174.

99 Ford AC, Forman D, Hunt R, et al. Helicobacter pylori eradication for the prevention of gastric neoplasia. Cochrane Database Syst Rev 2015;7:CD005583.

100 Doorakkers E, Lagergren J, Engstrand L, et al. Eradication of Helicobacter pylori and gastric cancer: a systematic review and meta-analysis of cohort studies. J Natl Cancer Inst 2016;108:djw132.

101 Lee YC, Chiang TH, Chou CK, et al. Association between Helicobacter pylori eradication and gastric cancer incidence: a systematic review and meta-analysis. Gastroenterology 2016;150:1113-24

102 O'Connor A, O'Morain CA, Ford AC. Population screening and treatment of Helicobacter pylori infection. Nat Rev Gastroenterol Hepatol 2017;14:230-40.

103 Pan KF, Zhang L, Gerhard M, et al. A large randomised controlled intervention trial to prevent gastric cancer by eradication of Helicobacter pylori in Linqu County, China: baseline results and factors affecting the eradication. Gut 2016;65:9-18.

104 Chen HN, Wang Z, Li X, et al. Helicobacter pylori eradication cannot reduce the risk of gastric cancer in patients with intestinal metaplasia and dysplasia: evidence from a meta-analysis. Gastric Cancer 2016;19:166-75.

105 Rokkas T, Rokka A, Portincasa P. A systematic review and meta-analysis of the role of Helicobacter pylori eradication in preventing gastric cancer. Ann Gastroenterol 2017;30:414-23

106 Choi IJ, Kook M-C, Kim Y-I, et al. Helicobacter pylori therapy for the prevention of metachronous gastric cancer. N Engl J Med Overseas Ed 2018;378:1085-95.

107 Goddard AF, Badreldin R, Pritchard DM, et al. British Society of Gastroenterology. The management of gastric polyps. Gut 2010;59:1270-6.

108 Ohkusa T, Miwa H, Hojo M, et al. Endoscopic, histological and serologic findings of gastric hyperplastic polyps after eradication of Helicobacter pylori: comparison between responder and non-responder cases. Digestion 2003;68:57-62.

109 Ohkusa T, Takashimizu I, Fujiki K, et al. Disappearance of hyperplastic polyps in the stomach after eradication of Helicobacter pylori. A randomized, clinical trial. Ann Intern Med 1998;129:712-5.

110 Ji F, Wang ZW, Ning JW, et al. Effect of drug treatment on hyperplastic gastric polyps infected with Helicobacter pylori: a randomized, controlled trial. World J Gastroenterol 2006;12:1770-3.

111 Ljubicić N, Banić M, Kujundzić M, et al. The effect of eradicating Helicobacter pylori infection on the course of adenomatous and hyperplastic gastric polyps. Eur $J$ Gastroenterol Hepatol 1999;11:727-30.

112 Huang YK, Yu JC, Kang WM, et al. Significance of serum pepsinogens as a biomarker for gastric cancer and atrophic gastritis screening: a systematic review and metaanalysis. PLoS One 2015;10:e0142080.

113 Yeh JM, Hur C, Ward Z, et al. Gastric adenocarcinoma screening and prevention in the era of new biomarker and endoscopic technologies: a cost-effectiveness analysis. Gut 2016:65:563-74.

114 Kuipers EJ. In through the out door: serology for atrophic gastritis. Eur J Gastroenterol Hepatol 2003;15:877-9.

115 Lomba-Viana R, Dinis-Ribeiro M, Fonseca F, et al. Serum pepsinogen test for early detection of gastric cancer in a European country. Eur J Gastroenterol Hepatol 2012;24:37-41.

116 Miki K, Fujishiro M, Kodashima S, et al. Long-term results of gastric cancer screening using the serum pepsinogen test method among an asymptomatic middle-aged Japanese population. Dig Endosc 2009;21:78-81.

117 Dinis-Ribeiro M, Yamaki G, Miki K, et al. Meta-analysis on the validity of pepsinogen test for gastric carcinoma, dysplasia or chronic atrophic gastritis screening. J Med Screen 2004;11:141-7.

118 Broutet N, Plebani M, Sakarovitch C, et al. Pepsinogen A, pepsinogen C, and gastrin as markers of atrophic chronic gastritis in European dyspeptics. $\mathrm{Br} J$ Cancer 2003;88:1239-47.

119 Terasawa T, Nishida H, Kato K, et al. Prediction of gastric cancer development by serum pepsinogen test and Helicobacter pylori seropositivity in Eastern Asians: a systematic review and meta-analysis. PLoS One 2014;9:e109783.

120 Yamaguchi Y, Nagata Y, Hiratsuka R, et al. Gastric cancer screening by combined assay for serum anti-Helicobacter pylori igG antibody and serum pepsinogen levelsthe abc method. Digestion 2016;93:13-18.

121 Lansdorp-Vogelaar I, Kuipers EJ. Screening for gastric cancer in Western countries. Gut 2016:65:543-4.

122 Ikeda F, Shikata K, Hata J, et al. Combination of Helicobacter pylori antibody and serum pepsinogen as a good predictive tool of gastric cancer incidence: 20-year prospective data from the Hisayama study. J Epidemiol 2016;26:629-36.

123 Zagari RM, Rabitti S, Greenwood DC, et al. Systematic review with meta-analysis: diagnostic performance of the combination of pepsinogen, gastrin-17 and antiHelicobacter pylori antibodies serum assays for the diagnosis of atrophic gastritis. Aliment Pharmacol Ther 2017;46:657-67.

124 McNicholl AG, Forné M, Barrio J, et al. Accuracy of GastroPanel for the diagnosis of atrophic gastritis. Eur J Gastroenterol Hepatol 2014;26:941-8.

125 Masci E, Pellicano R, Mangiavillano B, et al. GastroPanel $®$ test for non-invasive diagnosis of atrophic gastritis in patients with dyspepsia. Minerva Gastroenterol Dietol 2014;60:79-83.
126 Compare D, Rocco A, Nardone G. Screening for and surveillance of gastric cancer. World J Gastroenterol 2014:20:13681-91.

127 Amal H, Leja M, Funka K, et al. Detection of precancerous gastric lesions and gastric cancer through exhaled breath. Gut 2016:65:400-7.

128 Menon S, Trudgill N. How commonly is upper gastrointestinal cancer missed at endoscopy? A meta-analysis. Endosc Int Open 2014;2:E46-50.

129 Beg S, Ragunath K, Wyman A, et al. Quality standards in upper gastrointestinal endoscopy: a position statement of the British Society of Gastroenterology (BSG) and Association of Upper Gastrointestinal Surgeons of Great Britain and Ireland (AUGIS). Gut 2017:66:1886-99.

130 Bisschops R, Areia M, Coron E, et al. Performance measures for upper gastrointestinal endoscopy: a European Society of Gastrointestinal Endoscopy (ESGE) quality improvement initiative. Endoscopy 2016;48:843-64.

131 Teh JL, Tan JR, Lau LJ, et al. Longer examination time improves detection of gastric cancer during diagnostic upper gastrointestinal endoscopy. Clin Gastroenterol Hepatol 2015;13:480-7.

132 Teh JL, Hartman M, Lau L, et al. Mo1579 duration of endoscopic examination significantly impacts detection rates of neoplastic lesions during diagnostic upper endoscopy. Gastrointest Endosc 2011;73:AB393.

133 Taga S. Endoscopy technique. Observation by panendoscopy. In: Tada M, Maruyama M, Fujino M, eds. I to Cho Handbook. Tokyo: Igakushoin, 1992:132-9.

134 JSGCS. The committee for standardizing screening gastroscopy. Gastric cancer screeing techniques. Handbook. 1st ed. Tokyo: Igakushoin, 2010.

135 Yao K. The endoscopic diagnosis of early gastric cancer. Ann Gastroenterol 2013;26:11-22

136 Zhang Q, Chen Z, Chen C, et al. Training in early gastric cancer diagnosis improves the detection rate of early gastric cancer. Medicine (Baltimore) 2015:94:e384.

137 Yao K, Uedo N, Muto M, et al. Development of an e-learning system for teaching endoscopists how to diagnose early gastric cancer: basic principles for improving early detection. Gastric Cancer 2017;20:28-38

138 Yao K, Uedo N, Muto M, et al. Development of an e-learning system for the endoscopic diagnosis of early gastric cancer: an international multicenter randomized controlled trial. EBioMedicine 2016;9:140-7

139 Nakanishi $\mathrm{H}$, Doyama $\mathrm{H}$, Ishikawa $\mathrm{H}$, et al. Evaluation of an e-learning system for diagnosis of gastric lesions using magnifying narrow-band imaging: a multicenter randomized controlled study. Endoscopy 2017;49:957-67.

140 Yagi K, Aruga Y, Nakamura A, et al. Regular arrangement of collecting venules (RAC): a characteristic endoscopic feature of Helicobacter pylori-negative normal stomach and its relationship with esophago-gastric adenocarcinoma. J Gastroenterol 2005;40:443-52

141 Yagi $\mathrm{K}$, Honda $\mathrm{H}$, Yang JM, et al. Magnifying endoscopy in gastritis of the corpus. Endoscopy 2005:37:660-6.

142 Yagi K, Nakamura A, Sekine A. Comparison between magnifying endoscopy and histological, culture and urease test findings from the gastric mucosa of the corpus. Endoscopy 2002;34:376-81.

$143 \mathrm{Cho} \mathrm{JH}$, Chang YW, Jang JY, et al. Close observation of gastric mucosal pattern by standard endoscopy can predict Helicobacter pylori infection status. J Gastroenterol Hepatol 2013;28:279-84.

144 Na S, Chung JW, Park HJ, et al. [The usefulness of the regular arrangement of collecting venules pattern for the determination of Helicobacter pylori Infection]. Korean J Gastroenterol 2011;58:252

145 Machado RS, Viriato A, Kawakami E, et al. The regular arrangement of collecting venules pattern evaluated by standard endoscope and the absence of antrum nodularity are highly indicative of Helicobacter pylori uninfected gastric mucosa. Dig Liver Dis 2008;40:68-72.

146 Muto M, Yao K, Kaise M, et al. Magnifying endoscopy simple diagnostic algorithm for early gastric cancer (MESDA-G). Dig Endosc 2016;28:379-93.

147 Bansal A, Ulusarac O, Mathur S, et al. Correlation between narrow band imaging and nonneoplastic gastric pathology: a pilot feasibility trial. Gastrointest Endosc 2008:67:210-6.

148 Uedo N. Advanced imaging in the diagnosis of gastric intestinal metaplasia: the expert's approach. Video Journal and Encyclopedia of GI Endoscopy 2013:1:112-4.

149 Anagnostopoulos GK, Yao K, Kaye P, et al. High-resolution magnification endoscopy can reliably identify normal gastric mucosa, Helicobacter pylori-associated gastritis, and gastric atrophy. Endoscopy 2007:39:202-7.

150 Pimentel-Nunes P, Dinis-Ribeiro M, Soares JB, et al. A multicenter validation of an endoscopic classification with narrow band imaging for gastric precancerous and cancerous lesions. Endoscopy 2012;44:236-46

151 Gonen C, Simsek I, Sarioglu S, et al. Comparison of high resolution magnifying endoscopy and standard videoendoscopy for the diagnosis of Helicobacter pylori gastritis in routine clinical practice: a prospective study. Helicobacter 2009; 14:12-21.

152 Uedo N, Yao K. Endoluminal diagnosis of early gastric cancer and its precursors: bridging the gap between endoscopy and pathology. Adv Exp Med Biol 2016;908:293-316.

153 Yao K, Iwashita A, Tanabe H, et al. White opaque substance within superficial elevated gastric neoplasia as visualized by magnification endoscopy with narrow- 
band imaging: a new optical sign for differentiating between adenoma and carcinoma. Gastrointest Endosc 2008:68:574-80.

154 Nakayama Y, Horiuchi A, Kumagai T, et al. Discrimination of normal gastric mucosa from Helicobacter pylori gastritis using standard endoscopes and a single observation site: studies in children and young adults. Helicobacter 2004;9:95-9.

155 Redéen S, Petersson F, Jönsson KA, et al. Relationship of gastroscopic features to histological findings in gastritis and Helicobacter pylori infection in a general population sample. Endoscopy 2003;35:946-50.

156 Kimura K, Takemoto T. An endoscopic recognition of the atrophic border and its significance in chronic gastritis. Endoscopy 1969;1:87-97.

157 Naylor GM, Gotoda T, Dixon M, et al. Why does Japan have a high incidence of gastric cancer? Comparison of gastritis between UK and Japanese patients. Gut 2006;55:1545-52.

158 Kono S, Gotoda T, Yoshida S, et al. Can endoscopic atrophy predict histological atrophy? Historical study in United Kingdom and Japan. World I Gastroenterol 2015;21:13113-23

159 Nagata N, Shimbo T, Akiyama J, et al. Predictability of gastric intestinal metaplasia by mottled patchy erythema seen on endoscopy. Gastroenterology Res 2011:4:203-9.

160 Lim JH, Kim N, Lee HS, et al. Correlation between endoscopic and histological diagnoses of gastric intestinal metaplasia. Gut Liver 2013;7:41-50.

161 Panteris V, Nikolopoulou S, Lountou A, et al. Diagnostic capabilities of highdefinition white light endoscopy for the diagnosis of gastric intestinal metaplasia and correlation with histologic and clinical data. Eur I Gastroenterol Hepatol 2014;26:1-601.

162 Pimentel-Nunes P, Libânio D, Lage J, et al. A multicenter prospective study of the real-time use of narrow-band imaging in the diagnosis of premalignant gastric conditions and lesions. Endoscopy 2016;48:723-30.

163 Kikuste I, Stirna D, Liepniece-Karele I, et al. The accuracy of flexible spectral imaging colour enhancement for the diagnosis of gastric intestinal metaplasia: do we still need histology to select individuals at risk for adenocarcinoma? Eur J Gastroenterol Hepatol 2014;26:704-9.

164 Ang TL, Pittayanon R, Lau JY, et al. A multicenter randomized comparison between high-definition white light endoscopy and narrow band imaging for detection of gastric lesions. Eur I Gastroenterol Hepatol 2015;27:1473-8.

165 Buxbaum JL, Hormozdi D, Dinis-Ribeiro M, et al. Narrow-band imaging versus white light versus mapping biopsy for gastric intestinal metaplasia: a prospective blinded trial. Gastrointest Endosc 2017;86:857-65.

166 Uedo $\mathrm{N}$, Ishihara $\mathrm{R}$, lishi $\mathrm{H}$, et al. A new method of diagnosing gastric intestinal metaplasia: narrow-band imaging with magnifying endoscopy. Endoscopy 2006:38:819-24

167 An JK, Song GA, Kim GH, et al. Marginal turbid band and light blue crest, signs observed in magnifying narrow-band imaging endoscopy, are indicative of gastric intestinal metaplasia. BMC Gastroenterol 2012;12:169.

168 Kanemitsu T, Yao K, Nagahama T, et al. Extending magnifying NBI diagnosis of intestinal metaplasia in the stomach: the white opaque substance marker. Endoscopy 2017:49:529-35

169 Wang L, Huang W, Du J, et al. Diagnostic yield of the light blue crest sign in gastric intestinal metaplasia: a meta-analysis. PLoS One 2014;9:e92874.

170 Eshmuratov A, Nah JC, Kim N, et al. The correlation of endoscopic and histological diagnosis of gastric atrophy. Dig Dis Sci 2010;55:1364-75.

171 Zhang Q, Wang F, Chen ZY, et al. Comparison of the diagnostic efficacy of white light endoscopy and magnifying endoscopy with narrow band imaging for early gastric cancer: a meta-analysis. Gastric Cancer 2016;19:543-52.

172 Libânio D, Braga V, Ferraz S, et al. Prospective comparative study of endoscopic submucosal dissection and gastrectomy for early neoplastic lesions including patients' perspectives. Endoscopy 2019:51:30-9.

173 Axon A. Symptoms and diagnosis of gastric cancer at early curable stage. Best Pract Res Clin Gastroenterol 2006:20:697-708.

174 Coda S, Lee SY, Gotoda T. Endoscopic mucosal resection and endoscopic submucosal dissection as treatments for early gastrointestinal cancers in Western countries. Gut Liver 2007;1:012-21.

175 Endoscopic Classification Review Group. Update on the Paris classification of superficial neoplastic lesions in the digestive tract. Endoscopy 2005;37:570-8.

176 Veitch AM, Uedo N, Yao K, et al. Optimizing early upper gastrointestinal cancer detection at endoscopy. Nat Rev Gastroenterol Hepatol 2015;12:660-7.

177 Lauwers GY. Defining the pathologic diagnosis of metaplasia, atrophy, dysplasia, and gastric adenocarcinoma. J Clin Gastroenterol 2003:36:S37-43.

178 Park DY, Srivastava A, Kim GH, et al. Adenomatous and foveolar gastric dysplasia: distinct patterns of mucin expression and background intestinal metaplasia. Am J Surg Pathol 2008;32:524-33.

179 Correa P, Haenszel W, Cuello C, et al. Gastric precancerous process in a high risk population: cohort follow-up. Cancer Res 1990;50:4737-40.

180 Uedo N, lishi H, Tatsuta M, et al. Longterm outcomes after endoscopic mucosal resection for early gastric cancer. Gastric Cancer 2006;9:88-92.

181 Rokkas T, Filipe MI, Sladen GE. Detection of an increased incidence of early gastric cancer in patients with intestinal metaplasia type III who are closely followed up. Gut 1991;32:1110-3.
182 Whiting JL, Sigurdsson A, Rowlands DC, et al. The long term results of endoscopic surveillance of premalignant gastric lesions. Gut 2002:50:378-81.

183 den Hollander WJ, Holster IL, den Hoed CM, et al. Surveillance of premalignant gastric lesions: a multicentre prospective cohort study from low incidence regions. Gut 2019;68:585-93.

184 Yeh JM, Hur C, Kuntz KM, et al. Cost-effectiveness of treatment and endoscopic surveillance of precancerous lesions to prevent gastric cancer. Cancer 2010;116:2941-53.

185 Areia M, Dinis-Ribeiro M, Rocha Gonçalves F. Cost-utility analysis of endoscopic surveillance of patients with gastric premalignant conditions. Helicobacter 2014;19:425-36.

186 Zhou HJ, Dan YY, Naidoo N, et al. A cost-effectiveness analysis evaluating endoscopic surveillance for gastric cancer for populations with low to intermediate risk. PLoS One 2013;8:e83959.

187 Wu JT, Zhou J, Naidoo N, et al. Determining the cost-effectiveness of endoscopic surveillance for gastric cancer in patients with precancerous lesions. Asia Pac J Clin Oncol 2016:12:359-68.

188 Di Gregorio C, Morandi P, Fante R, et al. Gastric dysplasia. A follow-up study. Am J Gastroenterol 1993:88:1714-9.

189 Fertitta AM, Comin U, Terruzzi V, et al. Clinical significance of gastric dysplasia: a multicenter follow-up study. Gastrointestinal Endoscopic Pathology Study Group. Endoscopy 1993;25:265-8

190 Yamada H, Ikegami M, Shimoda T, et al. Long-term follow-up study of gastric adenoma/dysplasia. Endoscopy 2004:36:390-6.

191 Rugge M, Cassaro M, Di Mario F, et al. The long term outcome of gastric noninvasive neoplasia. Gut 2003:52:1111-6.

192 Park SY, Jeon SW, Jung MK, et al. Long-term follow-up study of gastric intraepithelial neoplasias: progression from low-grade dysplasia to invasive carcinoma. Eur $\rfloor$ Gastroenterol Hepatol 2008;20:966-70.

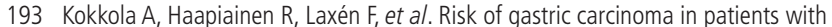
mucosal dysplasia associated with atrophic gastritis: a follow up study. J Clin Pathol 1996:49:979-84

194 Rugge M, Farinati F, Baffa R, et al. Gastric epithelial dysplasia in the natural history of gastric cancer: a multicenter prospective follow-up study. Interdisciplinary Group on Gastric Epithelial Dysplasia. Gastroenterology 1994;107:1288-96.

195 Simone A, Casadei A, De Vergori E, et al. Rescue endoscopy to identify site of gastric dysplasia or carcinoma found at random biopsies. Dig Liver Dis 2011:43:721-5

196 Abraham SC, Park SJ, Lee JH, et al. Genetic alterations in gastric adenomas of intestinal and foveolar phenotypes. Mod Pathol 2003;16:786-95.

197 Zhao G, Xue M, Hu Y, et al. How commonly is the diagnosis of gastric low grade dysplasia upgraded following endoscopic resection? A meta-analysis. PLoS One 2015:10:e0132699.

198 Kim YJ, Park JC, Kim JH, et al. Histologic diagnosis based on forceps biopsy is not adequate for determining endoscopic treatment of gastric adenomatous lesions. Endoscopy 2010;42:620-6

199 Nam KW, Song KS, Lee HY, et al. Spectrum of final pathological diagnosis of gastric adenoma after endoscopic resection. World J Gastroenterol 2011;17:5177-83.

200 Jeon HK, Ryu HY, Cho MY, et al. A randomized trial to determine the diagnostic accuracy of conventional vs. jumbo forceps biopsy of gastric epithelial neoplasias before endoscopic submucosal dissection; open-label study. Gastric Cancer 2014:17:661-8

201 Goo JJ, Choi CW, Kang DH, et al. Risk factors associated with diagnostic discrepancy of gastric indefinite neoplasia: who need en bloc resection? Surg Endosc 2015;29:3761-7.

202 Raftopoulos SC, Kumarasinghe P, de Boer B, et al. Gastric intraepithelial neoplasia in a Western population. Eur J Gastroenterol Hepatol 2012;24:48-54.

203 Pimentel-Nunes P, Mourão F, Veloso N, et al. Long-term follow-up after endoscopic resection of gastric superficial neoplastic lesions in Portugal. Endoscopy 2014:46:933-40.

204 Pyo JH, Lee H, Min BH, et al. Long-term outcome of endoscopic resection vs. surgery for early gastric cancer: a non-inferiority-matched cohort study. Am J Gastroenterol 2016:111:240-9.

205 Kosaka T, Endo M, Toya Y, et al. Long-term outcomes of endoscopic submucosal dissection for early gastric cancer: a single-center retrospective study. Dig Endosc 2014;26:183-91.

206 Kato M, Nishida T, Yamamoto K, et al. Scheduled endoscopic surveillance controls secondary cancer after curative endoscopic resection for early gastric cancer: a multicentre retrospective cohort study by Osaka University ESD study group. Gut 2013:62:1425-32

207 Horiki N, Omata F, Uemura M, et al. Risk for local recurrence of early gastric cance treated with piecemeal endoscopic mucosal resection during a 10-year follow-up period. Surg Endosc 2012;26:72-8.

208 Ahn JY, Jung HY, Choi JY, et al. Natural course of noncurative endoscopic resection of differentiated early gastric cancer. Endoscopy 2012;44:1114-20

209 Oda I, Gotoda T, Sasako M, et al. Treatment strategy after non-curative endoscopic resection of early gastric cancer. Br J Surg 2008;95:1495-500 
210 Yoon H, Kim SG, Choi J, et al. Risk factors of residual or recurrent tumor in patients with a tumor-positive resection margin after endoscopic resection of early gastric cancer. Surg Endosc 2013;27:1561-8

211 Pimentel-Nunes P, Dinis-Ribeiro M, Ponchon T, et al. Endoscopic submucosal dissection: European Society of Gastrointestinal Endoscopy (ESGE) Guideline. Endoscopy 2015;47:829-54

212 Japanese Gastric Cancer Association. Japanese gastric cancer treatment guidelines 2010 (ver. 3). Gastric Cancer 2011:14:113-23.

213 Japanese Gastric Cancer Association. Japanese gastric cancer treatment guidelines 2014 (ver. 4). Gastric Cancer 2017:20:1-19.

214 Lauren P. The two histological main types of gastric carcinoma: diffuse and so-called intestinal-type carcinoma. An attempt at a histo-clinical classification. Acta Pathol Microbiol Scand 1965;64:31-49.

215 Qiu MZ, Cai MY, Zhang DS, et al. Clinicopathological characteristics and prognostic analysis of Lauren classification in gastric adenocarcinoma in China. J Trans/ Med 2013;11:58

216 Gotoda T, Yanagisawa A, Sasako M, et al. Incidence of lymph node metastasis from early gastric cancer: estimation with a large number of cases at two large centers. Gastric Cancer 2000:3:219-25.

217 Hirasawa T, Gotoda T, Miyata S, et al. Incidence of lymph node metastasis and the feasibility of endoscopic resection for undifferentiated-type early gastric cancer. Gastric Cancer 2009;12:148-52.

218 Hasuike N, Ono H, Boku N, et al. A non-randomized confirmatory trial of an expanded indication for endoscopic submucosal dissection for intestinal-type gastric cancer (cT1a): the Japan Clinical Oncology Group study (JCOG0607). Gastric Cancer 2018:21:114-23.

219 Probst A, Schneider A, Schaller T, et al. Endoscopic submucosal dissection for early gastric cancer: are expanded resection criteria safe for Western patients? Endoscopy 2017;49:855-65.

220 Hatta W, Gotoda T, Oyama T, et al. A scoring system to stratify curability after endoscopic submucosal dissection for early gastric cancer: "eCura system". Am J Gastroentero/ 2017;112:874-81.

221 Facciorusso A, Antonino M, Di Maso M, et al. Endoscopic submucosal dissection vs endoscopic mucosal resection for early gastric cancer: a meta-analysis. World J Gastrointest Endosc 2014:6:555-63.

222 Takekoshi T, Baba Y, Ota H, et al. Endoscopic resection of early gastric carcinoma: results of a retrospective analysis of 308 cases. Endoscopy 1994;26:352-8.

223 Hiki Y, Shimao H, Mieno H, et al. Modified treatment of early gastric cancer: evaluation of endoscopic treatment of early gastric cancers with respect to treatment indication groups. World J Surg 1995; 19:517-22.

224 Nagano H, Ohyama S, Fukunaga T, et al. Indications for gastrectomy after incomplete EMR for early gastric cancer. Gastric Cancer 2005;8:149-54.

225 Tanabe S, Ishido K, Higuchi K, et al. Long-term outcomes of endoscopic submucosal dissection for early gastric cancer: a retrospective comparison with conventional endoscopic resection in a single center. Gastric Cancer 2014;17:130-6.

226 Oda I, Gotoda T, Hamanaka H, et al. Endoscopic submucosal dissection for early gastric cancer: technical feasibility, operation time and complications from a large consecutive series. Digestive Endoscopy 2005;17:54-8.

227 Lian J, Chen S, Zhang Y, et al. A meta-analysis of endoscopic submucosal dissection and EMR for early gastric cancer. Gastrointest Endosc 2012;76:763-70.

228 Park YM, Cho E, Kang HY, et al. The effectiveness and safety of endoscopic submucosal dissection compared with endoscopic mucosal resection for early gastric cancer: a systematic review and metaanalysis. Surg Endosc 2011:25:2666-77.

229 Ono H, Yao K, Fujishiro M, et al. Guidelines for endoscopic submucosal dissection and endoscopic mucosal resection for early gastric cancer. Dig Endosc 2016;28:3-15.

230 Farhat $\mathrm{S}$, Chaussade $\mathrm{S}$, Ponchon T, et al. Endoscopic submucosal dissection in a European setting. A multi-institutional report of a technique in development. Endoscopy 2011:43:664-70.

231 Wu CY, Wu MS, Kuo KN, et al. Effective reduction of gastric cancer risk with regular use of nonsteroidal anti-inflammatory drugs in Helicobacter pylori-infected patients. J Clin Oncol 2010:28:2952-7.

232 Tian W, Zhao Y, Liu S, et al. Meta-analysis on the relationship between nonsteroidal anti-inflammatory drug use and gastric cancer. Eur J Cancer Prev 2010;19:288-98.

233 Wang WH, Huang JQ, Zheng GF, et al. Non-steroidal anti-inflammatory drug use and the risk of gastric cancer: a systematic review and meta-analysis. J Nat/ Cancer Inst 2003;95:1784-91.

234 Leung WK, Ng EK, Chan FK, et al. Effects of long-term rofecoxib on gastric intestinal metaplasia: results of a randomized controlled trial. Clin Cancer Res 2006:12:4766-72.

235 Yanaoka K, Oka M, Yoshimura N, et al. Preventive effects of etodolac, a selective cyclooxygenase-2 inhibitor, on cancer development in extensive metaplastic gastritis, a Helicobacter pylori-negative precancerous lesion. Int J Cancer 2010:126:1467-73.

236 Hung KH, Yang HB, Cheng HC, et al. Short-term celecoxib to regress long-term persistent gastric intestinal metaplasia after Helicobacter pylori eradication. $J$ Gastroenterol Hepatol 2010;25:48-53.
237 Yang HB, Cheng HC, Sheu BS, et al. Chronic celecoxib users more often show regression of gastric intestinal metaplasia after Helicobacter pylori eradication. Aliment Pharmacol Ther 2007;25:455-61.

238 Zhang LJ, Wang SY, Huo XH, et al. Anti-Helicobacter pylori therapy followed by celecoxib on progression of gastric precancerous lesions. World J Gastroenterol 2009:15:2731-8

239 Akre K, Ekström AM, Signorello LB, et al. Aspirin and risk for gastric cancer: a population-based case-control study in Sweden. Br J Cancer 2001;84:965-8.

240 Huang XZ, Chen Y, Wu J, et al. Aspirin and non-steroidal anti-inflammatory drugs use reduce gastric cancer risk: a dose-response meta-analysis. Oncotarget 2017:8:4781-95.

241 Correa P, Fontham ET, Bravo JC, et al. Chemoprevention of gastric dysplasia: randomized trial of antioxidant supplements and anti-Helicobacter pylori therapy. $J$ Natl Cancer Inst 2000;92:1881-8

242 You WC, Brown LM, Zhang L, et al. Randomized double-blind factorial trial of three treatments to reduce the prevalence of precancerous gastric lesions. J Nat/ Cancer Inst 2006:98:974-83.

243 Plummer M, Vivas J, Lopez G, et al. Chemoprevention of precancerous gastric lesions with antioxidant vitamin supplementation: a randomized trial in a high-risk population. J Nat/ Cancer Inst 2007;99:137-46.

244 Ferlay J. Cancer incidence and mortality worldwide. Ervik M, ed. Lyon: France: International Agency for Research on Cancer, 2013.

245 Hamashima C, Ogoshi K, Narisawa R, et al. Impact of endoscopic screening on mortality reduction from gastric cancer. World J Gastroentero/ 2015;21:2460-6.

246 Hamashima C, Shabana M, Okada K, et al. Mortality reduction from gastric cancer by endoscopic and radiographic screening. Cancer Sci 2015;106:1744-9.

247 Hosokawa O, Shinbo T, Matsuda K, et al. Impact of opportunistic endoscopic screening on the decrease of mortality from gastric cancer. J Gastroint Cancer Screen 2011;106:401-7.

248 Riecken B, Pfeiffer R, Ma JL, et al. No impact of repeated endoscopic screens on gastric cancer mortality in a prospectively followed Chinese population at high risk. Prev Med 2002;34:22-8

249 Matsumoto S, Yamasaki K, Tsuji K, et al. Results of mass endoscopic examination for gastric cancer in Kamigoto Hospital, Nagasaki Prefecture. World J Gastroenterol 2007:13:4316-20.

250 Matsumoto S, Yoshida Y. Efficacy of endoscopic screening in an isolated island: a case-control study. Indian J Gastroenterol 2014;33:46-9.

251 Hamashima C, Ogoshi K, Okamoto M, et al. A community-based, case-control study evaluating mortality reduction from gastric cancer by endoscopic screening in Japan. PLoS One 2013;8:e79088.

252 Hosokawa O, Hattori M, Takeda T, et al. Accuracy of endoscopy in detecting gastric cancer. J Gastroenterol Mass Surv 2004;14:33-9.

253 Choi KS, Jun JK, Park EC, et al. Performance of different gastric cancer screening methods in Korea: a population-based study. PLoS One 2012;7:e50041.

254 Hamashima C, Okamoto M, Shabana M, et al. Sensitivity of endoscopic screening for gastric cancer by the incidence method. Int J Cancer 2013;133:653-9.

255 Otsuji M, Kouno Y, Otsuji A, et al. Assessment of small diameter panendoscopy for diagnosis of gastric cancer: comparative study with follow-up survey date. Stomach Intest 1989;24:1291-7.

256 Gupta N, Bansal A, Wani SB, et al. Endoscopy for upper GI cancer screening in the general population: a cost-utility analysis. Gastrointest Endosc 2011;74:610-24.

257 Lee YC, Lin JT, Wu HM, et al. Cost-effectiveness analysis between primary and secondary preventive strategies for gastric cancer. Cancer Epidemiol Biomarkers Prev 2007:16:875-85.

258 Areia M, Spaander MC, Kuipers EJ, et al. Endoscopic screening for gastric cancer: a cost-utility analysis for countries with an intermediate gastric cancer risk. United European Gastroenterol J 2018;6:205064061772290.

259 Shawihdi M, Thompson E, Kapoor N, et al. Variation in gastroscopy rate in English general practice and outcome for oesophagogastric cancer: retrospective analysis of Hospital Episode Statistics. Gut 2014;63:250-61.

260 Leung WK, Ho HJ, Lin JT, et al. Prior gastroscopy and mortality in patients with gastric cancer: a matched retrospective cohort study. Gastrointest Endosc 2018;87:119-27.

261 Carmack SW, Genta RM, Graham DY, et al. Management of gastric polyps: a pathology-based guide for gastroenterologists. Nat Rev Gastroenterol Hepatol 2009:6:331-41.

262 Evans JA, Chandrasekhara V, Chathadi KV, et al. The role of endoscopy in the management of premalignant and malignant conditions of the stomach. Gastrointest Endosc 2015;82:1-8

263 Graham JR. Gastric polyposis: onset during long-term therapy with omeprazole. Med J Aust 1992:157:287-8.

264 Chandrasekhara V, Ginsberg GG. Endoscopic management of gastrointestinal stromal tumors. Curr Gastroenterol Rep 2011;13:532-9.

265 Islam RS, Patel NC, Lam-Himlin D, et al. Gastric polyps: a review of clinical, endoscopic, and histopathologic features and management decisions. Gastroenterol Hepatol 2013;9:640-51

266 Han JS, Jang JS, Choi SR, et al. A study of metachronous cancer after endoscopic resection of early gastric cancer. Scand J Gastroenterol 2011;46:1099-104 
267 Ahn JY, Son DH, Choi KD, et al. Neoplasms arising in large gastric hyperplastic polyps: endoscopic and pathologic features. Gastrointest Endosc 2014;80:1005-13.

268 Kang HM, Oh TH, Seo JY, et al. [Clinical factors predicting for neoplastic transformation of gastric hyperplastic polyps]. Korean I Gastroenterol 2011;58:184-9.

269 Daibo M, Itabashi M, Hirota T. Malignant transformation of gastric hyperplastic polyps. Am J Gastroenterol 1987;82:1016-25.

270 Dirschmid K, Platz-Baudin C, Stolte M. Why is the hyperplastic polyp a marker for the precancerous condition of the gastric mucosa? Virchows Arch 2006;448:80-4.

271 Ginsberg GG, Al-Kawas FH, Fleischer DE, et al. Gastric polyps: relationship of size and histology to cancer risk. Am J Gastroenterol 1996;91:714-7.

272 Rattan J, Arber N, Tiomny E, et al. Gastric polypoid lesions-an eight-year study. Hepatogastroenterology 1993;40:107-9.

273 Hizawa K, Fuchigami T, lida M, et al. Possible neoplastic transformation within gastric hyperplastic polyp. Application of endoscopic polypectomy. Surg Endosc 1995;9:714-8.

274 Zea-Iriarte WL, Sekine I, Itsuno M, et al. Carcinoma in gastric hyperplastic polyps. A phenotypic study. Dig Dis Sci 1996;41:377-86.

275 Cristallini EG, Ascani S, Bolis GB. Association between histologic type of polyp and carcinoma in the stomach. Gastrointest Endosc 1992:38:481-4.

276 Harju E. Gastric polyposis and malignancy. Br I Surg 1986;73:532-3.

277 Markowski AR, Markowska A, Guzinska-Ustymowicz K. Pathophysiological and clinical aspects of gastric hyperplastic polyps. World I Gastroenterol 2016:22:8883-91.

278 Uedo N, Yamaoka R, Yao K. Multiple white flat lesions in the gastric corpus are not intestinal metaplasia. Endoscopy 2017:49:615-6.

279 Hasegawa R, Yao K, Ihara S, et al. Magnified endoscopic findings of multiple white flat lesions: a new subtype of gastric hyperplastic polyps in the stomach. Clin Endosc 2018;51:558-62.

280 Han AR, Sung CO, Kim KM, et al. The clinicopathological features of gastric hyperplastic polyps with neoplastic transformations: a suggestion of indication for endoscopic polypectomy. Gut Liver 2009;3:271-5.

281 Stolte M, Sticht T, Eidt S, et al. Frequency, location, and age and sex distribution of various types of gastric polyp. Endoscopy 1994;26:659-65.

282 Laxén F, Sipponen P, Ihamäki T, et al. Gastric polyps; their morphological and endoscopical characteristics and relation to gastric carcinoma. Acta Pathol Microbiol Immunol Scand A 1982;90:221-8.

283 Omori T, Kamiya Y, Tahara T, et al. Correlation between magnifying narrow band imaging and histopathology in gastric protruding/or polypoid lesions: a pilot feasibility trial. BMC Gastroenterol 2012;12:17.

284 Kanesaka T, Sekikawa A, Tsumura T, et al. Dense-type crypt opening seen on magnifying endoscopy with narrow-band imaging is a feature of gastric adenoma. Dig Endosc 2014;26:57-62.

285 Yao K, Iwashita A, Nambu M, et al. Nature of white opaque substance in gastric epithelial neoplasia as visualized by magnifying endoscopy with narrow-band imaging. Dig Endosc 2012;24:419-25.

286 Colling C, Asztalos I, Buchner AM, et al. Mo2037 narrow-band imaging classification system for gastric polyps may reduce the need for biopsies. Gastrointest Endosc 2017;85:AB531.
287 Muehldorfer SM, Stolte M, Martus P, et al. Diagnostic accuracy of forceps biopsy versus polypectomy for gastric polyps: a prospective multicentre study. Gut 2002;50:465-70.

288 Brown H, Wyatt S, Croft S, et al. An evaluation of endoscopycapacity across the NHS in England. London: Cancer Research UK, 2015.

289 Ross-Innes CS, Debiram-Beecham I, O'Donovan M, et al. Evaluation of a minimally invasive cell sampling device coupled with assessment of trefoil factor 3 expression for diagnosing Barrett's esophagus: a multi-center case-control study. PLoS Med 2015:12:e1001780.

290 Dinis-Ribeiro M, da Costa-Pereira A, Lopes C, et al. Magnification chromoendoscopy for the diagnosis of gastric intestinal metaplasia and dysplasia. Gastrointest EndosC 2003;57:498-504.

291 Guo YT, Li YQ, Yu T, et al. Diagnosis of gastric intestinal metaplasia with confocal laser endomicroscopy in vivo: a prospective study. Endoscopy 2008;40:547-53.

292 Tahara T, Shibata T, Nakamura M, et al. Gastric mucosal pattern by using magnifying narrow-band imaging endoscopy clearly distinguishes histological and serological severity of chronic gastritis. Gastrointest Endosc 2009; 70:246-53

293 Capelle LG, Haringsma J, de Vries AC, et al. Narrow band imaging for the detection of gastric intestinal metaplasia and dysplasia during surveillance endoscopy. Dig Dis Sci 2010:55:3442-8.

294 Kawamura M, Abe S, Oikawa K, et al. Topographic differences in gastric micromucosal patterns observed by magnifying endoscopy with narrow band imaging. J Gastroenterol Hepatol 2011;26:477-83.

295 Rerknimitr R, Imraporn B, Klaikeaw N, et al. Non-sequential narrow band imaging for targeted biopsy and monitoring of gastric intestinal metaplasia. World J Gastroenterol 2011;17:1336-42.

296 Dutta AK, Sajith KG, Pulimood AB, et al. Narrow band imaging versus white light gastroscopy in detecting potentially premalignant gastric lesions: a randomized prospective crossover study. Indian I Gastroenterol 2013;32:37-42.

297 Kanzaki H, Uedo N, Ishihara R, et al. Comprehensive investigation of areae gastricae pattern in gastric corpus using magnifying narrow band imaging endoscopy in patients with chronic atrophic fundic gastritis. Helicobacter 2012:17:224-31.

298 Savarino E, Corbo M, Dulbecco P, et al. Narrow-band imaging with magnifying endoscopy is accurate for detecting gastric intestinal metaplasia. World I Gastroenterol 2013;19:2668-75.

299 Fukuta N, Ida K, Kato T, et al. Endoscopic diagnosis of gastric intestinal metaplasia: a prospective multicenter study. Dig Endosc 2013;25:526-34.

300 Pittayanon R, Rerknimitr R, Wisedopas N, et al. Flexible spectral imaging color enhancement plus probe-based confocal laser endomicroscopy for gastric intestinal metaplasia detection. J Gastroenterol Hepatol 2013;28:1004-9.

301 Xirouchakis E, Laoudi F, Tsartsali L, et al. Screening for gastric premalignant lesions with narrow band imaging, white light and updated Sydney protocol or both? Dig Dis Sci 2013;58:1084-90.

302 Nomura S, Ida K, Terao S, et al. Endoscopic diagnosis of gastric mucosal atrophy: multicenter prospective study. Dig Endosc 2014;26:709-19.

303 Sha J, Wang P, Zhu B, et al. Acetic acid enhanced narrow band imaging for the diagnosis of gastric intestinal metaplasia. PLoS One 2017;12:e0170957. 